Felipe Warwar Murad

\title{
Performance evaluation of nonlinear explicit algebraic Reynolds stress models to predict channel flows
}

Dissertação de Mestrado

Dissertation presented to the Programa de Pós-graduação em Engenharia Mecânica of PUC-Rio in partial fulfillment of the requirements for the degree of Mestre em Engenharia Mecânica. Approved by the undersigned Examination Committee.

Advisor: Angela Ourivio Nieckele

Co-Advisor: Luiz Eduardo Bittencourt Sampaio

Rio de Janeiro

July 2018 


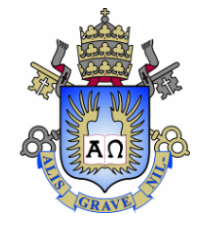

Felipe Warwar Murad

\section{Performance evaluation of nonlinear explicit algebraic Reynolds stress models to predict channel flows}

Dissertation presented to the Programa de Pósgraduação em Engenharia Mecânica of PUC-Rio in partial fulfillment of the requirements for the degree of Mestre em Engenharia Mecânica. Approved by the undersigned Examination Committee.

Profa. Angela Ourivio Nieckele Advisor Departamento de Engenharia Mecânica - PUC-RIO

Dr. Luiz Eduardo Bittencourt Sampaio Co-Advisor Department of Energy Resources Engineering - Stanford University

Prof. Roney Leon Thompson Departamento de Engenharia Mecânica - UFRJ

Prof. Aristeu da Silveira Neto Centro de Ciências Exatas e Tecnologia - UFU

Prof. Márcio da Silveira Carvalho

Vice Dean of Graduate Studies Centro Técnico Científico - PUC-RIO

Rio de Janeiro, July 24th, 2018 
All rights reserved

Felipe Warwar Murad

The author graduated in Mechanical Engineering from Pontifícia Universidade Católica do Rio de Janeiro (PUC-RJ) in July 2016 with additional domain in software development

Ficha Catalográfica

Murad, Felipe Warwar

Performance evaluation of nonlinear explicit algebraic Reynolds stress models to predict channel flows / Felipe Warwar Murad ; advisor: Angela Ourivio Nieckele ; coadvisor: Luiz Eduardo Bittencourt Sampaio. - 2018.

$91 \mathrm{f}$. : il. color. ; $30 \mathrm{~cm}$

Dissertação (mestrado)-Pontifícia Universidade Católica do Rio de Janeiro, Departamento de Engenharia Mecânica, 2018.

Inclui bibliografia

1. Engenharia Mecânica - Teses. 2. Turbulência. 3. Modelos RANS lineares e não lineares. 4. Escoamento em um canal. 5. Modelo de viscosidade turbulenta. I. Nieckele, Angela Ourivio. II. Sampaio, Luiz Eduardo Bittencourt. III. Pontifícia Universidade Católica do Rio de Janeiro. Departamento de Engenharia Mecânica. IV. Título. 


\section{Acknowlegment}

I would like to thank first my advisor, Angela Ourivio Nieckele, who indicated me to the subject of this work. Without her (as an advisor and a teacher) this work would not be concluded. I also thank professor Roney Thompson and professor Luiz Eduardo Sampaio who offer support to achieve the results.

To the CNPq institute, for the financial support provided during the last two years in which this work was developed.

To my family: my father Marcelo, mother Carla, sister Flávia and grandparents Munir and Reneé. I am extremely grateful for the education, dedication and affection which was provided through my whole life.

I save one special paragraph to my uncle and aunt Marcio Murad and Elisa Massa who also offered support to my career in every difficult moment.

To my girlfriend Desirre Mazzarella whose affection, understanding and persistence in my personal and professional growth cannot be left aside. I thank her not only for the trust in these 6 years but also for your support on the hard times.

Finally, I am grateful for all institutions and companies that were part of my path as a student and an Engineer. I am especially grateful to Santo Agostinho school and PUC-Rio to provide an exceptional learning environment and to GT2 Energia and TECGRAF to teach me how to be an engineer in practice. 


\section{Abstract}

Murad, Felipe Warwar; Sampaio, Luiz Eduardo Bittencout (Co-Advisor); Nieckele, Angela Ourivio (Advisor). Performance evaluation of nonlinear explicit algebraic Reynolds stress models to predict channel flows. Rio de Janeiro, 2018. 91p. Dissertação de Mestrado - Departamento de Engenharia Mecânica, Pontifícia Universidade Católica do Rio de Janeiro.

The most popular models to solve turbulent flows are based on the Reynolds Average Navier Stokes approach (RANS), which needs closure equations to relate the Reynolds stress tensor to the mean kinematic tensors. The classical approach is the Boussinesq approximation that assumes a linear relation between the deviatoric part of the Reynolds stress tensor, and the rate of strain tensor. Previous works have shown, that the non-linear dependence on the rate of strain tensor can improve the model predictions. At the present work, first an evaluation of linear models available in the literature is performed, followed by the analysis of three higher order methods, that expands the tensorial basis to include other objective orthogonal tensors. Two different nondimensionalization, one with the turbulent kinetic energy and dissipation rate and the other one with turbulent kinetic energy and the intensity of the rate of strain, had also been proposed for the models. The performance of the new models is assessed by comparing their numerical predictions to available channel flow and for a broad range of Reynolds Numbers. All models are implemented in the open source platform OpenFOAM. Reasonable predictions of the Reynolds shear component of all models were obtained when compared with the DNS data. However, the non-linear models proved superior in the prediction of the other components. It was also observed that the model which depends nonlinearly with the rate of strain and linearly with the non-persistence of strain was the one that best represented the DNS data field.

\section{Keywords}

Turbulence; Linear and Non-Linear RANS Models; Channel Flow; Eddy Viscosity Turbulence Model 


\section{Resumo}

Murad, Felipe Warwar. Sampaio, Luiz Eduardo Bittencout; Nieckele, Angela Ourivio. Avaliação de desempenho de modelos explícitos algébricos não lineares de tensões de Reynolds para previsão de escoamentos em canais. Rio de Janeiro, 2018. 91p. Dissertação de Mestrado - Departamento de Engenharia Mecânica, Pontifícia Universidade Católica do Rio de Janeiro.

Os modelos mais populares para solucionar escoamentos turbulentos são baseados no esquema RANS (Reynolds Average Navier Stokes) que necessita de fechamento para relacionar o tensor de tensões de Reynolds com os tensores médios cinemáticos. A solução clássica é a aproximação por Bussinesq que assume uma relação linear entre a parte deviatórica do Tensor de Reynolds e o tensor das taxas de deformação. Trabalhos anteriores mostraram que uma relação não linear entre o tensor das taxas de deformação pode melhorar a predição do modelo. No presente trabalho, primeiramente é realizada uma avaliação entre modelos lineares presentes na literatura seguido de uma análise de três modelos de ordem elevada que expandem a base tensorial para incluir tensores ortogonais. Duas adimensionalizações, uma com a energia cinética turbulenta e taxa de dissipação e outra com energia cinética turbulenta e intensidade do tensor de deformação, haviam sido propostas. As previsões dos modelos são comparados com dados DNS para um canal e para uma gama variada de número de Reynolds. Todos os modelos são implementados na plataforma aberta OpenFoam. Predições razoáveis para a componente cisalhante de todos os modelos foram obtidas quando comparadas com os dados DNS. Entretanto, modelos não lineares provaram superioridade na predição das outras componentes. Também foi observado que o modelo não linearmente dependente do tensor taxa de deformação e o tensor não persistencia das deformações foi o que melhor representou os campos providos por DNS.

\section{Palavras-chave:}

Turbulência; Modelos RANS Lineares e Não Lineares; Escoamento em um Canal; Modelo de Viscosidade Turbulenta 


\section{Contents}

1 Introduction 14

1.1 Objetive 3

1.2 Manuscript Organization 3

2 Literature Review 20

3 Mathematical and Numerical Modeling 28

3.1 Mathematical Modeling: RANS Methodology 28

3.2 The Boussinesq Hypothesis 30

$\begin{array}{lll}3.2 .1 & \text { The } \boldsymbol{\kappa}-\boldsymbol{\varepsilon} \text { Model } & 31\end{array}$

3.2.2 Linear Low Reynolds K- $\varepsilon$ Models 33

3.3 Non-Linear Models 34

3.3.1 The $\mathrm{K}-\varepsilon$ Lien Cubic Model 36

3.3.2 Non-Linear Models Based on Orthogonal Decomposition 36

3.3.3 Non-linear $\boldsymbol{\kappa}-\boldsymbol{\varepsilon}$ Models 38

$\begin{array}{lll}\text { 3.3.4 Non-linear } \boldsymbol{\kappa}-\boldsymbol{\gamma} \text { Models } & 40\end{array}$

3.4 Numerical Model 41

4 Results $\quad 45$

$\begin{array}{lll}4.1 & \text { Reference Velocity Field } & 47\end{array}$

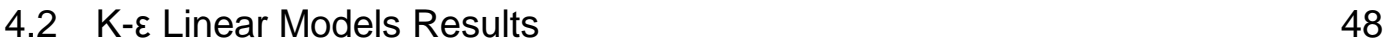

4.3 K-ع Non- Linear Models' Results 51

4.3.1 Models II, III and IV Results

4.3.2 Comparison with Literature Non-Linear Model 55

4.3.3 Models' Results for Different Reynolds Numbers 57

4.4 K-ү Models' Results 63

5 Conclusion $\quad 70$ 
5.1 Future Work 71

$\begin{array}{ll}\text { Bibliography } & 73\end{array}$

Appendix A - Coefficients Adjustments for $\kappa-\varepsilon$ Models 76

A.1 Coefficient $\boldsymbol{C \mu}$

A.2 Damping functions of non-linear terms $\boldsymbol{f} \boldsymbol{\mu} \mathbf{2}$ and $\boldsymbol{f} \boldsymbol{\beta}$

Appendix B - Coefficients Adjustments for $\kappa-\gamma$ Models 81

B.1 Coefficient $\boldsymbol{C \mu}-\boldsymbol{\gamma}$

B.2 Coefficient $C \boldsymbol{\mu 2}-\boldsymbol{\gamma}$

B.3 Coefficient $\boldsymbol{C} \boldsymbol{\beta}-\boldsymbol{\gamma}$

Appendix C - Mesh Considerations $\quad 87$

$\begin{array}{ll}\text { C.1 Mesh Distribution } & 87\end{array}$

C.2 Mesh Refinement 88

Appendix D - Influence of $\mathrm{K}$ and $\varepsilon$ field into $\mathrm{K}-\varepsilon$ NLEVM 90 
Figure List

Figure 1.1 - Laminar Transition and Turbulent Zones Source: (Michael et al., 2014)

Figure 1.2 - Sketch of Vortex Stretching in a Wind Tunnel Source: (Tennekes \& Lumley, 1972)

Figure $2.1-\mathrm{k}+$ and $\varepsilon+$ Comparison of Low Reynolds Models. Source Michelassi et al. (1993)

Figure 2.2 - Velocity and Reynolds Components of the Abe et al. (2003) model I for a Channel flow of RE=6875 SOURCE: ABE ET AL. (2003) .

Figure 2.3 - Velocity and Reynolds Components of the Abe et al. (2003) model II for a channel flow of $R e=6875$ Source: Abe et al. (2003).

Figure 2. 4 - Velocity and Reynolds Components of the Abe et al. (2003) model III for a channel flow of $R e=6875$ Source: Abe et al. (2003)

Figure 2.5 - A priori analysis of the six models proposed by (Nieckele et al., 2016) in a Channel Flow with $\operatorname{Re} \tau=1000$.

Figure 3.1 - Near wall classification regions. Adapted from (Pope, 2000)............27

Figure 4.1 - Simulation's Geometry 45

Figure 4.2 - Comparison of Velocity Profile from the DNS data with Reference Field for $\mathrm{RE}_{\mathrm{T}}=1000$

Figure 4.3 - Comparison of the mean axial velocity. Linear Models. $\operatorname{Re} \tau=1000 \ldots 49$

Figure 4.4 - Rodi Mansour Comparison with Linear an logarithmic behavior. ...... 49

Figure 4.5 - Comparison of shear Reynolds stress. Linear Models. $\operatorname{Re\tau }=1000 \ldots 50$

Figure 4.6 - Comparison of turbulent kinetic energy and its dissipation. Linear Models. $\operatorname{Re\tau }=1000$

Figure 4.7 - Comparison of mean axial velocity. Non-Linear Models. $\boldsymbol{R e \tau}=1000$.

Figure 4.8 - Reynolds tensor components. Non-Linear Models. $\operatorname{Re\tau }=1000$.........54

Figure 4.9 - Turbulent kinetic energy and its dissipation. Non-Linear Models.

$\operatorname{Re} \tau=1000$

Figure 4.10 - A priori analysis of the six models proposed by (Nieckele et al., 2016) in a Channel Flow with $\operatorname{Re} \tau=1000$.

Figure 4.11 - Comparison of mean axial velocity. Model III versus Lien Cubic model. $\boldsymbol{R} \boldsymbol{e} \tau=1000$. 
Figure 4.12 - Reynolds tensor components. Model III versus Lien Cubic model.

$\operatorname{Re\tau }=1000$

Figure 4.13 - Turbulent kinetic energy and its dissipation. MODEL III VERSUS Lien Cubic model. $\operatorname{Re} \tau=1000$

Figure 4.14 - Comparison of mean axial velocity with DNS data. Different Reynolds numbers.

Figure 4.15 - Comparison of the shear Reynolds tensor components axy with DNS data. Different Reynolds numbers.

Figure 4.16-Comparison of the normal Reynolds tensor components $\operatorname{axx}$ and ayy with DNS data. Different Reynolds numbers.

Figure 4.17 - Comparison of the normal Reynolds tensor components azz with DNS data. Different Reynolds numbers.

Figure 4.18 - Comparison of turbulent kinetic energy and its dissipation with DNS data. Different Reynolds numbers.

Figure 4.19 - Model III Reynolds Stress for different Reynolds Number .66

Figure 4.20 - Comparison of models $\kappa-\gamma$ and $\kappa-\varepsilon$ models for the mean axial velocity and shear Reynolds stress component.

Figure 4.21 - Comparison of models $\mathrm{k}-\mathrm{y}$ and $\mathrm{k}-\varepsilon$ models for the normal Reynolds stress component, axx and ayy

Figure 4.22 - Comparison of models $\mathrm{k}-\mathrm{y}$ and $\mathrm{k}-\varepsilon$ models for the normal Reynolds stress component, azz

Figure 4.23 - Comparison of models $k-\gamma$ and $k-\varepsilon$ models for the turbulent kinetic energy and its dissipation.

Figure A.1 - Shear Reynolds tensor component axy with Rodi Mansour Modified (RMM) damping function, with different $C \mu$

Figure A.2 Mean axial velocity with Rodi Mansour Modified (RMM) damping function, with different $C \mu$

Figure A.3 $-\mathrm{K}$ and $\varepsilon$ with Rodi Mansour Modified (RMM) damping function, with different $C \mu$.

Figure A.4 - Damping functions of the non-linear terms from Nieckele et al. (2016)

Figure A.5 - New damping functions of the non-linear terms. .79

Figure A.6 - Reynolds Stress Comparison from different damping functions ........80

Figure B.1 $-\boldsymbol{C} \boldsymbol{\mu}-\boldsymbol{\gamma}$ from DNS for different $\boldsymbol{R e \tau}$ : 395, 590, $1000 \ldots \ldots \ldots \ldots \ldots \ldots \ldots \ldots . . . . . . \ldots 1$

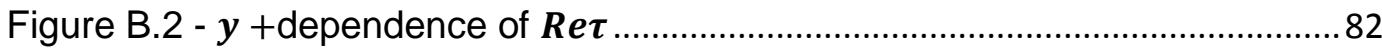

Figure B.3 - Fitting of $\boldsymbol{C} \boldsymbol{\mu}-\boldsymbol{\gamma} \boldsymbol{y}+<\boldsymbol{y}+$ at different Reynolds Numbers ....................83

Figure B.4 - "a" parameter as a function of the Reynolds number .84 
Figure B.5 $-\boldsymbol{C} \boldsymbol{\mu}-\boldsymbol{\gamma}$ all regions integrated and compared with the dns data.........84

Figure B.6 - Coefficient and $D^{2}$ term comparison using DNS data .........................8 85

Figure B.7 - Coefficient and P term comparison using DNS data............................86

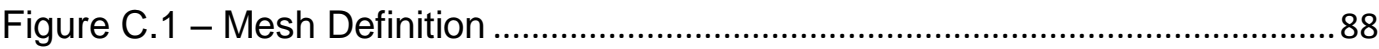

Figure C. 2 - Mean axial velocity Model III $R e_{\tau}=1000$. Mesh Test ........................8 88

Figure C.3 - Reynolds Stress Tensor Components. Model III. $R e_{\tau}=1000$. Mesh

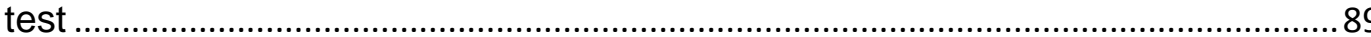

Figure C.3 - Turbulent kinetic energy and dissipation. Model III. $R e_{\tau}=$

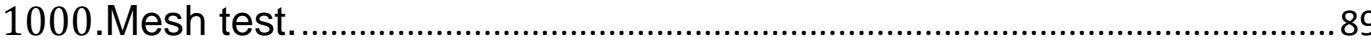

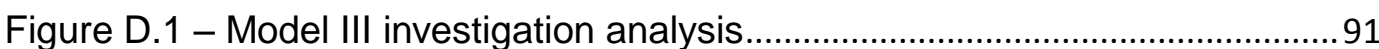




\section{Symbols}

\begin{tabular}{|c|c|c|}
\hline & $\kappa$ & Mean turbulent kinetic energy \\
\hline & $\varepsilon$ & Mean rate of dissipation of the turbulent energy \\
\hline & $a$ & Deviatoric Reynolds stress tensor \\
\hline & $x$ & Axial direction \\
\hline & $y$ & Normal direction \\
\hline & $u^{*}$ & Velocity \\
\hline & $p^{*}$ & Pressure \\
\hline & $D^{*}$ & Rate of strain tensor \\
\hline & $\mathrm{T}$ & Time \\
\hline & $p$ & Mean pressure \\
\hline$\sqrt{n}$ & $\mu$ & Viscosity \\
\hline$\widetilde{\sigma}$ & $v$ & Kinematic viscosity \\
\hline$\stackrel{2}{\Xi}$ & $\rho$ & Density \\
\hline$\vec{\theta}$ & $g$ & Gravity \\
\hline 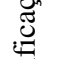 & $D$ & Mean Rate of strain tensor \\
\hline U. & $U$ & Mean velocity \\
\hline$\stackrel{\frac{0}{2}}{\frac{\pi}{1}}$ & $\tau_{w}$ & Shear tension at the wall \\
\hline & $u_{\tau}$ & Friction velocity \\
\hline & $\mu_{t}$ & Turbulent viscosity \\
\hline & $V_{c}$ & Characteristic velocity \\
\hline & $l_{c}$ & Characteristic length \\
\hline & $R e$ & Reynolds Number \\
\hline & $R e_{\tau}$ & Friction Reynolds number \\
\hline & $G_{\kappa}$ & Production term \\
\hline & $\tilde{\varepsilon}$ & pseudo epsilon \\
\hline & $k$ & Von-karma number \\
\hline & $f_{\mu}, f_{\mu 2}, f_{\beta}, f_{1}, f_{2}$ & Damping functions \\
\hline & $N L S$ & Non-linear stress additional terms \\
\hline
\end{tabular}




$\begin{array}{ll}P & \text { Non-persistence of strain tensor } \\ W^{*} & \text { Vorticity written into rate of strain eigenvectors' frame } \\ W & \text { Vorticity } \\ \Omega_{i j}^{D} & \text { Rate of strain eigenvectors' tensor } \\ a_{D 0}, a_{D}, a_{D 1}, a_{D 2}, a_{\beta} & \text { Dimensional models' coefficients } \\ C_{\mu}, C_{\mu 2}, C_{\beta} & \text { Nondimensional } \kappa-\varepsilon \text { models' coefficients } \\ C_{\mu-\gamma}, C_{\mu 2-\gamma}, C_{\beta-\gamma} & \text { Nondimensional } \kappa-\gamma \text { models' coefficients } \\ \dot{\gamma} & \text { Rate of strain intensity } \\ H & \text { Half channel's distance } \\ \beta & \text { Channel's imposed pressure gradient } \\ A & \text { Original Tensor } \\ \mathrm{B} & \text { Decomposition Tensor } \\ \hat{y}^{+} & y^{+} a t \text { the maximum } C_{\mu-\gamma} \text { value }\end{array}$

Subscript

$i, j, k \quad$ indexes

superscript

Perturbation

Orthogonal Tensor

Wall coordinates 


\section{Introduction}

Turbulence flows can be found in nature and in many engineering applications. Some examples are wake turbulence on aircraft wings, oil recovery and marine transportation, jet flows applications (for example to reduce noise or enhance mixing), dynamic scraped surface heat exchanger, combustion, gas turbines, meteorological predictions and marine currents path prediction. As it greatly affects different areas and industries, the correct comprehension and estimative of its dynamical and thermic behavior is of major importance.

The criteria often used to identify if a flow is laminar or turbulent is the Reynolds Number: the ratio of inertia and viscous forces. When the viscous forces dominate (low Reynolds number), any perturbation that might appear is damped by the viscous force, and the flow is laminar. However, when the inertia forces dominate (high Reynolds numbers) flow perturbations are amplified, small eddies are formed, the diffusion is substantially increased, and the flow is threedimensional (3D) and transient. The flow is then called turbulent. It is not possible to determine a unique Reynolds number value for the transition as stated by Trinh (2010), since the onset of turbulence can be offset by carefully controlling flow instabilities

Great attention in the past years have been given not only to determine the point where the transition regime would occur but also what would be the main characteristics of the flow until the turbulence stage.

To illustrate the flow's behavior from a laminar state until the turbulence's breakdown figure 1.1. shows a direct numerical simulation of the swept Hiemenz flow (present in swept wings for example) done by Michael et al. (2014). In this example, the perturbation is set as a pair of two counter vortices. At the first stage (inflow), the flow is laminar and no velocity instabilities are present. Moving along with it, the first instabilities (linear) in the velocity can be observed. They, later, develop into second order non-linear instabilities. After that, similar structures (coherent structures) and vortexes start to form until the turbulence breakdown. 


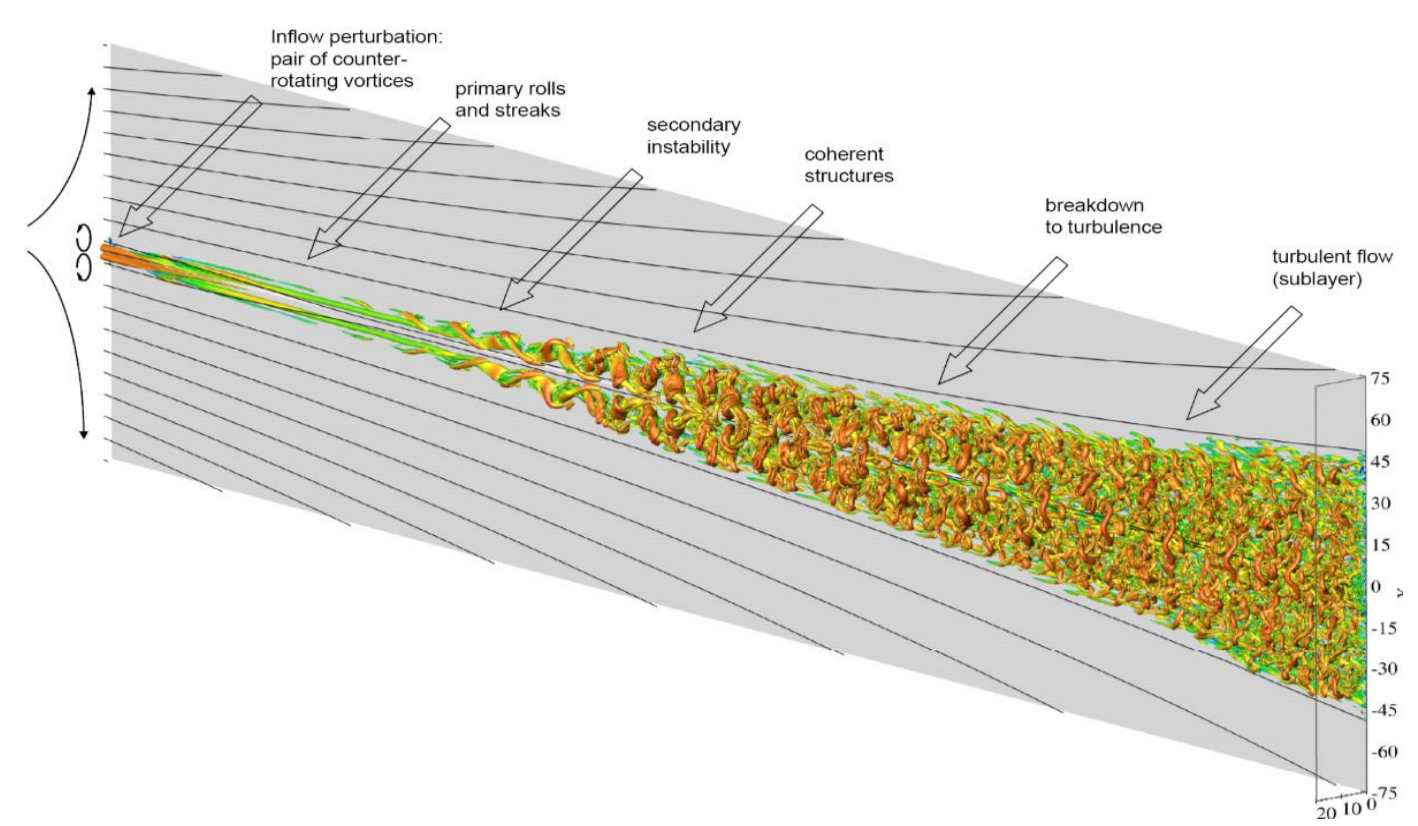

Figure 1.1 - Laminar Transition and Turbulent Zones Source: (Michael et al., 2014)

Several studies of the transition zone can be found in the literature for different geometries. He \& Seddinghi (2015) have investigated different transition Reynolds numbers in a channel. Gloor et al, (2016) investigated the linear instability for a jet flow Hosseinverdi \& Boroomand (2010) tried to predict the laminar, transition and turbulent flows in an airfoil using linear stability theory and $\mathrm{k}-\omega$ turbulence model.

Turbulence is also a diffusive phenomenon, therefore, mixing processes benefit from it. Also, this is the reason why streamline separations in an airfoil at high attack angle is prevented. Bilger (1992) addresses the mixing advantages brought by turbulent flows.

Turbulent flows are also dissipative. This means that it needs an outside energy source provider or else it will cease. The energy is injected on bigger scales and it is transferred to small scales, where it is dissipated. This process is often called the energy cascade as described by Pope (2000).

Turbulence only occurs in three dimensional rotational flows as it requires the presence of the vortex stretching phenomenon (Tennekes \& Lumley, 1972), which is illustrated by Fig.1.2. in a wind-tunnel contraction. The contraction is responsible to stretch the vortex which produces a higher angular velocity. Without this, vorticity would fade out and turbulence would cease. Since the vortex stretching phenomena cannot occur in $2 \mathrm{D}$ or $1 \mathrm{D}$ geometries, turbulence is only present in 3D flows. 


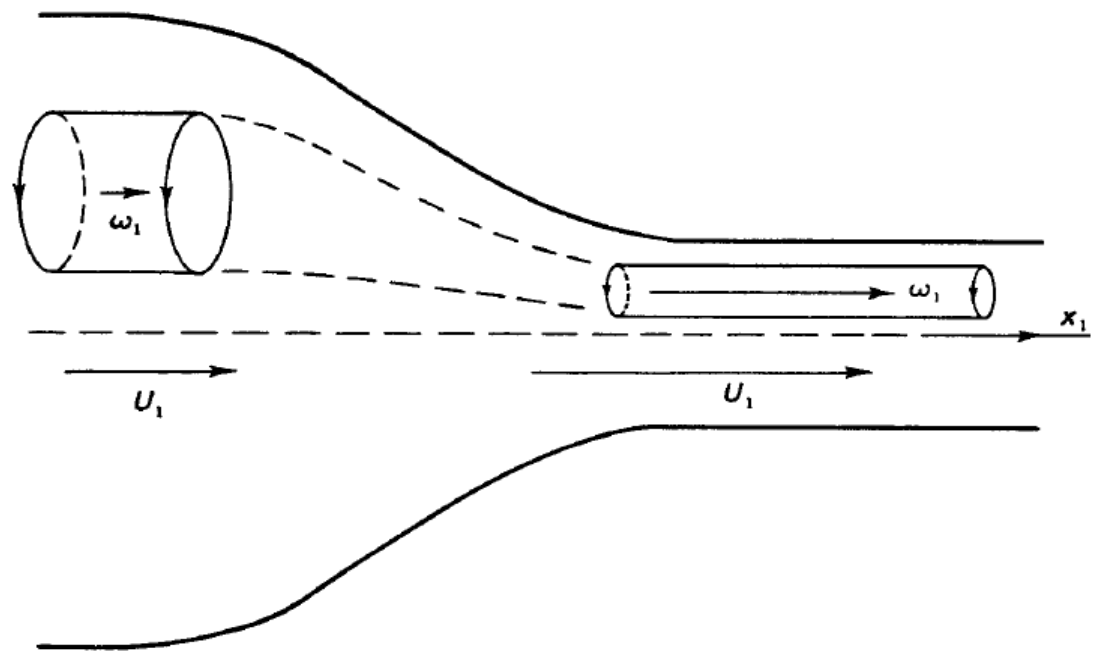

Figure 1.2 - Sketch of Vortex Stretching in a Wind Tunnel Source: (TENNEKES \& LUMLEY, 1972)

Due to its complexity, turbulence flows are calculated using numerical methods. Kolmogorov showed that the characteristic length and time scales of the turbulent flow are inversely proportional to the flow Reynolds Number (Pope, 2000). As a consequence, to be able to capture all flow relevant information, refined mesh and small time steps are necessary. Therefore, due to computing limitation, to allow prediction of the flow, different methodologies of turbulence simulation have been developed. The most popular methodologies, in a decreasing order of computational resources demand are:

- DNS (Direct Numerical Simulation): all scales are solved using the 3D transient momentum and continuity equations in a well-refined mesh with a small time step

- LES (Large Eddy Simulation): large eddies are solved in 3D transient momentum and continuity equations, and the small eddies are modeled.

- RANS (Reynolds-Averaged Navier-Stokes Simulation): all temporal scales are modeled and obtained by solving a time average momentum and continuity equations.

DNS approach was impossible to be done until 1970 due to computational capability. It is the simplest of the approaches and the one with best accuracy. From the given methodologies, DNS is the one which demands more computational power and it is still impractical to be employed from the industrial perspective. For this reason, this methodology is mostly employed for scientific purposes to 
understand the turbulence phenomena as well as for model's developing or calibration (usually for RANS models). For this purpose, several DNS databases for simple geometries, with different Reynolds numbers, are available in the literature.

With RANS approach, only time average results are obtained, and all scales are modeled. RANS approach has low computing demand, both with regard to memory and time requirements. Although it presents reasonable results in several situations, there are several phenomena that this approach is not able to capture. Further, the majority of the models within this approach are tuned for a particular class of problems.

LES approach requires an intermediary level of computational processing and presents an intermediate level of accuracy. This methodology is also transient and $3 \mathrm{D}$, but since its demand for mesh resolution is not as strict as with DNS, it has been used as an alternative for DNS for more difficult flows (more complex geometries and higher Reynolds number) and in situations where RANS cannot achieve good results. Although it is mainly applied for academic purposes, is beginning to be applied in some specific industrial problems. Because it also demands a high computing effort, LES methodology cannot and will not substitute RANS in the near future for industrial purposes (Zhiyin, 2014).

RANS is currently the most used methodology in the industry. Despite its practical use, standard RANS simulations cannot predict secondary and tertiary flows for more complex geometries. This methodology is based on the concept that only the statistically steady state properties are relevant, leading to the definition of the Reynolds Stress tensor. Many different models arise from this methodology (Pope, 2000). The Reynolds Stress tensor can be directly modeled or the turbulent viscosity concept can be introduced. The turbulent viscosity models vary from algebraic (Prandtl Mixing Length Model) to differential models (such as $\kappa-\varepsilon, \kappa-\varepsilon$ RNG, $\kappa-\omega, \kappa-\omega$ SST, v2f for example). Each one of them is based in distinguished fields used to calculate parameters of the flow, for instance, the Reynolds Tensor and/or turbulent viscosity.

Aiming to apply RANS approach in industrial application, there is a large effort from the scientific community to improve the prediction accuracy of RANS models. Recently, non-linear RANS models have been proposed, based on an orthogonal tensor decomposition of the Reynolds Stress on mean kinematic 
quantities, aiming to capture accurately not only the time average velocity profile, but also the Reynolds stress tensor (Nieckele et al., 2016). The models were developed applying noble available experimental and DNS data, and they were nondimensionalized applying the turbulent kinetic energy $\kappa$ and its dissipation rate $\varepsilon$. The authors presented a priori analysis of the models' performance indicating the superiority of the non-linear models. Here, a priori analysis means that the noble data was directly inserted into the models to evaluate the prediction of the Reynolds stress tensor.

\subsection{Objetive}

The main objective of the present work is to perform a posteriori analysis of the performance of the proposed models of Nieckele et al. (2016) to predict the flow field between two parallel plates. The posteriori analysis means that the mass and momentum equations are numerically solved coupled with turbulent quantities transport equations to determine the mean velocity field and the Reynolds stress tensor. To evaluate the models, the predictions are compared with the DNS data and different available linear and non-linear models.

At the present work, the coefficients nondimensionalization of the non-linear models proposed by Nieckele et al. (2016) are also evaluated, and new nondimensionalizations are proposed based on the turbulent kinetic energy $\kappa$ and the intensity of the rate of strain tensor $\gamma$ (Alves et al., 2014). Each set of models' results are analyzed together in order to estipulate which is the best combination of nondimensionalization parameters.

\subsection{Manuscript Organization}

This work is divided in six chapters. The first one is dedicated to state the objective, motivation and problem which is debated through this document.

In the second one, major advance in the turbulence modeling field is discussed. This chapter majorly focus on the RANS methodology, discussing about the major advances in linear and non-linear models, but also in DNS.

The third one presents the mathematical and numerical modeling that were used in the simulations. The RANS framework is overviewed as well as $\kappa-\varepsilon$ model equations. Existing Low Reynolds models (linear and non-linear) are also discussed 
in detail. The models used in the simulations, moreover, are presented. Finally, numerical details used in the simulations are shown.

In the fourth, the simulation's results are discussed. Each model is compared with existing models mentioned on Chapter 2. Result's analysis and further considerations of the proposed models are, furthermore, discussed.

Fifth Chapter summarizes the work's contributions for the turbulence modeling area as well as suggests possible advances for future works.

Appendixes A and B comment about the coefficients adjustments required in both $\kappa-\varepsilon$ and $\kappa-\gamma$ models. Appendix $C$ discusses the mesh influence in the results while appendix D explains the influence of $\kappa$ and $\varepsilon$ fields into the $\kappa-\varepsilon$ models results. 


\section{Literature Review}

This chapter presents a literature review related to development of the present work. As mentioned in Chapter 1, the main objective of this work is to access the performance of RANS models by comparing the prediction with high quality data. This type of data can be obtained by physical experimentation or with high quality numerical simulating employing DNS.

Three types of flow often analyzed with DNS that can be found in the literature are channel flow, free boundary and backstep flow. At the present work, the channel flow was selected. For the channel flow, a broad number of different Reynolds DNS simulations databases can be found. In this work databases from Lee \& Moser, (2015), who carried the same study for higher fiction Reynolds up to 5186 and Thais et al. (2012), who performed simulations of low friction Reynolds (180 to 1000) for Newtonian and viscoelastic fluids.

Other DNS simulations for the channel are reported by Bernardini et al. (2014) and Lozano-Durán \& Jiménez, (2014). Bernardini et al. (2014) executed simulations for low, moderate and moderate-high friction Reynolds (from 595 to 4079 ) in order to observe the logarithm behavior of the mean velocity profile. Meanwhile, Lozano-Durán \& Jiménez, (2014) studied the effect of the domain size in the DNS simulation for a friction Reynolds up to 4179.

Thompson et al. (2016) also analyzed a great number of channel DNS databases by comparing the DNS velocity field with the velocity field obtained from the averaged momentum equation using the DNS Reynolds Tensor and found out that, for some cases, these velocity profiles could not be correctly matched, especially for high friction Reynolds.

The RANS scheme is the most commonly used method in the industry due to its low computational cost. In this methodology, the time averaged Navier Stokes equation is taken into consideration. This allows to solve 3D flows such as jets or Channel/duct flows using 2D domains or even in steady state formulation. However, with the time average process, new unknown terms appear needing additional closure. Linear models follow the Boussinesq hypothesis which express 
the Reynolds stress tensor as a linear function of the rate of strain. The coefficient of this relation is called eddy viscosity.

Channel flow is often employed to evaluate the prediction capacity of a turbulent flow. In the text book of Wilcox, (2006) a comparison of the prediction of two algebraic turbulent models developed by Baldwin-Lomax (Baldwin \& Lomax, 1978) and Cebeci-Smith (Smith \& Cebeci, 1967) for a channel flow is presented for a Reynolds Number equal to 13750. It was shown that both models presented a close agreement to the DNS data. The Baldwin-Lomax model's result, however, performs better than Cebeci-Smith - 8\% of relative error for Cebeci-Smith and $5 \%$ for Baldwin-Lomax, when compared to the DNS. For all other quantities, the models agreed well.

The $\kappa-\varepsilon$ models are the most popular two-equation models in the RANS methodology and are available in most CFD softwares such as ANSYS Fluent and OpenFoam. The formulation of the model was first idealized by Harlow \& Nakayama (1968) while the main contribution was done by Jones \& Launder, (1972). The biggest problem, of these models is the derivation of the dissipation rate $\varepsilon$ equation, which has several terms that need closure, therefore, they are modeled with the addition of several empirical terms, see Rodi \& Mansour, (1993) for instance.

Another type of $\kappa-\varepsilon$ models (called Realizable $\kappa-\varepsilon$ ) was developed by Shih $e t$ al., (1994) by redefining the eddy viscosity and consequently the dissipation equation. The major advantage of this formulation was to avoid negative values of the Reynolds stress tensor when shear rate was high. As a result, spreading rate of round jets and reattachment points of a backward facing step could be better predicted.

One more $\kappa-\varepsilon$ variant ( $\kappa-\varepsilon$ RNG) introduced by Yakhot et al., (1992) determine a similar model with different coefficients. The model is derived with the usage of the Renormalization Group Theory.

Normally two equations models do not perform well near walls. To help the correct prediction in the whole domain, since the near wall region is critical to the flow, a possible alternative is the use of wall laws. These laws impose the log law to the closest mesh point to the surface. Care must be taken, however, for special conditions of the flow or the surface (pressure gradient, surface roughness and 
surface mass injection for example). For these situations the law of wall will need to be reformulated (Schlichting, 1979).

Another solution for this problem is the use of damping functions in the eddy viscosity and $\varepsilon$ equation. It requires that the near wall mesh is fine enough to capture the peak in the $\kappa$ field, or in other words, that the grid size, based Reynolds number, is low near the wall. For this reason, the formulation is referred to as a low Reynolds model, even when simulating a flow with a large Reynolds number - this one, based on a physical rather than grid related quantity.

The standard $\kappa-\omega$ model is the second most popular model, and it can be classified as low Reynolds number model. It can predict well boundary-layer flows and flows with adverse pressure gradient in streamwise direction since the region near the wall is well modeled. The formulation, however, imposes a sensible nonphysical fixed boundary condition at the wall.

To correct this problem, Menter, (1994) inserted a mixing function to the model. The idea behind its application was to switch between $\kappa-\omega$ (near the wall) and $\kappa-\varepsilon$ (away from the wall) so the benefits of these two models would be fully applicable in the simulation. The mixing function is formulated such that its value is one near the wall (so the behavior tends to $\kappa-\omega$ ) and zero away from the wall (tending to $\kappa-\varepsilon$ ). This formulation is known as $\kappa-\omega \mathrm{SST}$.

Another example of low Reynolds number models is the Launder-Sharma (Launder et al., 1977) model, which was first developed for spinning disc flow, but it is highly used for other geometries. Lien \& Leschziner, (1993) also evaluated a model with simulations for shock in a channel and nozzle geometries. Lam \& Bremhorst, (1981) proposed a low Reynolds model which shown good results for both turbulence and laminar flows. Furthermore, Rodi \& Mansour, (1993) formulated new damping functions based on DNS data to model the eddy viscosity. The authors also proposed new expressions to evaluate the terms of the $\varepsilon$ equation.

Michelassi et al. (1993) proposed modifications of some parameters in the Rodi Mansour's (Rodi \& Mansour, 1993) $\varepsilon$ equation, and, thus, formulated a similar model denominated Rodi Mansour Modified. The model was, then, simulated for a fully developed channel flow and boundary layer flow. The results were, furthermore, compared with other low Reynolds methods for a friction Reynolds of 395.

Figure 2.1 shows the results of Michelassi et al. (1993) in which their 
proposed model was compared with Launder Sharma (LS), Lam Bremhorst (LB) and Michelassi and Shin (MS) along with DNS data. It is possible to observe that the Rodi Mansour modified model could better predict the behavior of dimensionless dissipation $\varepsilon^{+}$near the wall relatively to other models, when compared with DNS data. For the dimensionless kinetic energy $\kappa^{+}$the Launder Sharma's result was the worst compared with the other models. The Lam Bremhorst and Michelassi and Shin underestimates this quantity while the Rodi Mansour Modified overestimates it.
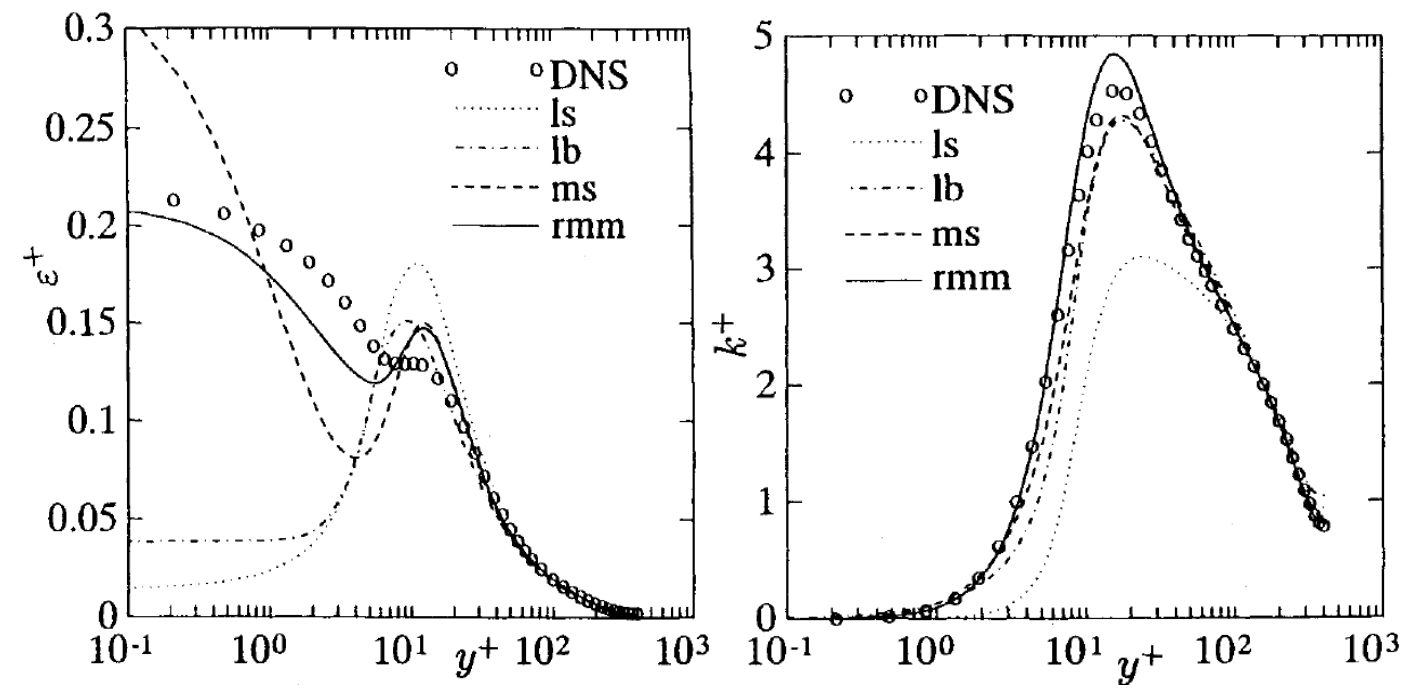

Figure $2.1-\mathrm{k}+$ and $\varepsilon+$ Comparison of low Reynolds Models Source: Michelassi et al. (1993)

One possible way to further enhance the RANS prediction methodology is to work directly with the Reynolds Stress Tensor, developing transport equations for each component. This approach requires additional closures, and a significant increase in cost, since besides the six Reynolds Stress components equations, the dissipation equation must also be solved.

A further step toward more complex and complete models, first introduced by Lumley, (1970) and Pope, (1975), is to relax the linearity constraint to implement non- linear eddy viscosity model (NLEVM). In this class of models, non-linear terms are added to the Boussinesq Hypothesis aiming at better predicting all components of the Reynolds Tensor. These terms are written based on a set of elected tensors to compound a more complete tensor basis. It is not desirable that this base choice compromises the frame-invariance of the model. The selected tensor should also carry the vorticity influence since it is well observed in all 
turbulence flows. Because of that, the vorticity tensor is often used to generate this tensor basis.

Another way to find a non-linear expression for the Reynolds Stress tensor is by using the weak equilibrium hypothesis (Sharath, 1996) that leads to an algebraic approximation of the equation that can be used. This class of model is called Explicit Algebraic Reynolds stress model (EARSM). More complex cases of EARMS models can be seen in Lazeroms et al. (2013) (stratified flow in a channel) and Grigoriev et al. (2015) (density influence in a turbulent flow in a subsonic and supersonic nozzle)

One challenge that both NLEVM and EARMS models face is due to the lack of frame invariance. Frame invariance is desirable property of a model which determines that its predictions are not affected by the chosen frame of coordinates. There is no agreement in which way the weak equilibrium hypothesis should be adapted in order to account for it. This question is further investigated by Hamba (2006), who showed a weak equilibrium condition which is frame-invariant. Many similarities and differences on NLEVM and EARMS models can be seen in Gatski \& Jongen, (2000).

Following this non-linear route, Lien et al. (1991) chose the vorticity and the rate of strain tensors as a basis for the Reynolds Tensor and created a low Reynolds NLEVM. Zhang et al. (2012) also used the same tensor bases and realizable conditions to analyze the result's difference of their NLEVM when compared to linear models for flows over a bluff body. As a result, they found that the developed non-linear model could better predict the flow.

Abe et al. (2003) proposed two $\kappa-\varepsilon$ NLEVM models (with different coefficients) and a variant $\kappa-\omega$ model which were tested and compared for a channel flow $(\operatorname{Re}=6875)$, a periodic channel with an obstacle $(\operatorname{Re}=21200)$ and a jet $(\operatorname{Re}=6000)$. Figure 2.2 through 2.4 shows the results (velocity profile and Reynolds Tensor) of the three proposed models for the channel flow case. Good agreement can be observed when compared to the DNS. For the $\kappa-\omega$ based model figure 2.4), the maximum velocity is a little underestimated. Also, for all cases, some difference can be noticed in the $u u^{+}$component away from the wall and in the $v v^{+}$component along all the domain. In this latter case, the prediction is consistently over estimated. 
Park et al. (2003) also proposed a non-linear near-wall model in which damping functions were used to express the linear and non-linear terms near the wall (similar to the low Reynolds Models). This damping function is derived, in part, from the Reynolds transport equation with homogeneous flow and local equilibrium state. The model's results are compared with DNS data from a channel and a backstep.
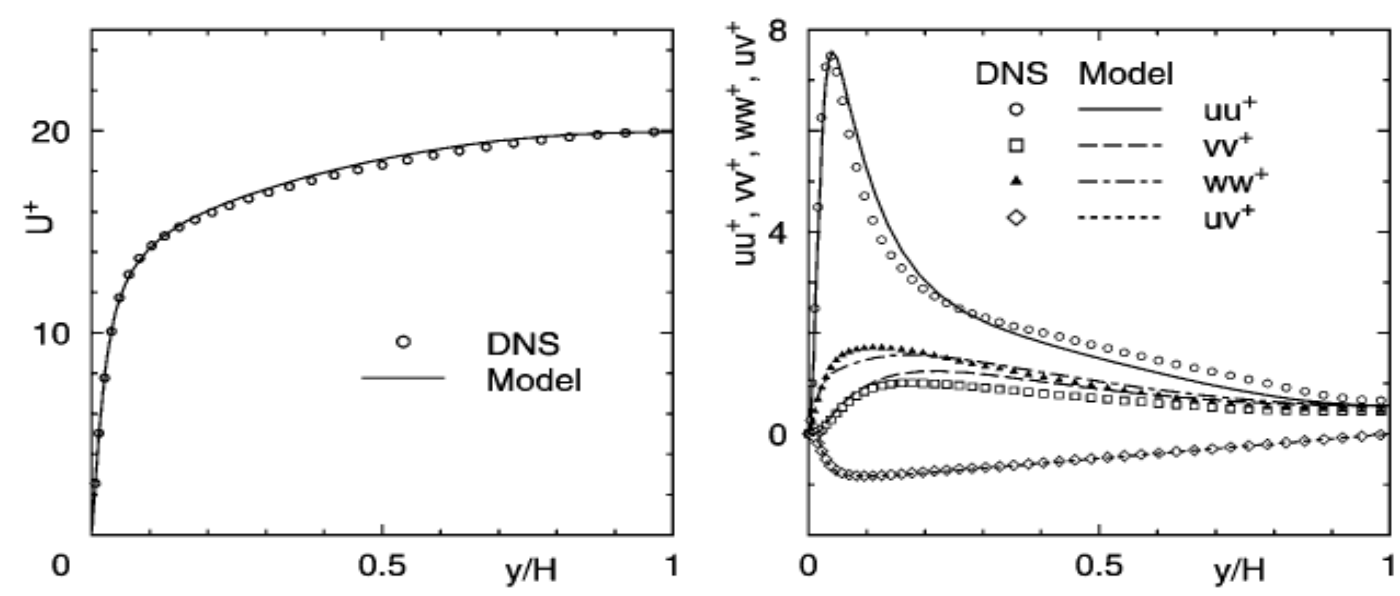

Figure 2.2 - Velocity and Reynolds Components of the Abe et al. (2003) model I for a channel flow of $R e=6875$ Source: Abe et al. (2003)
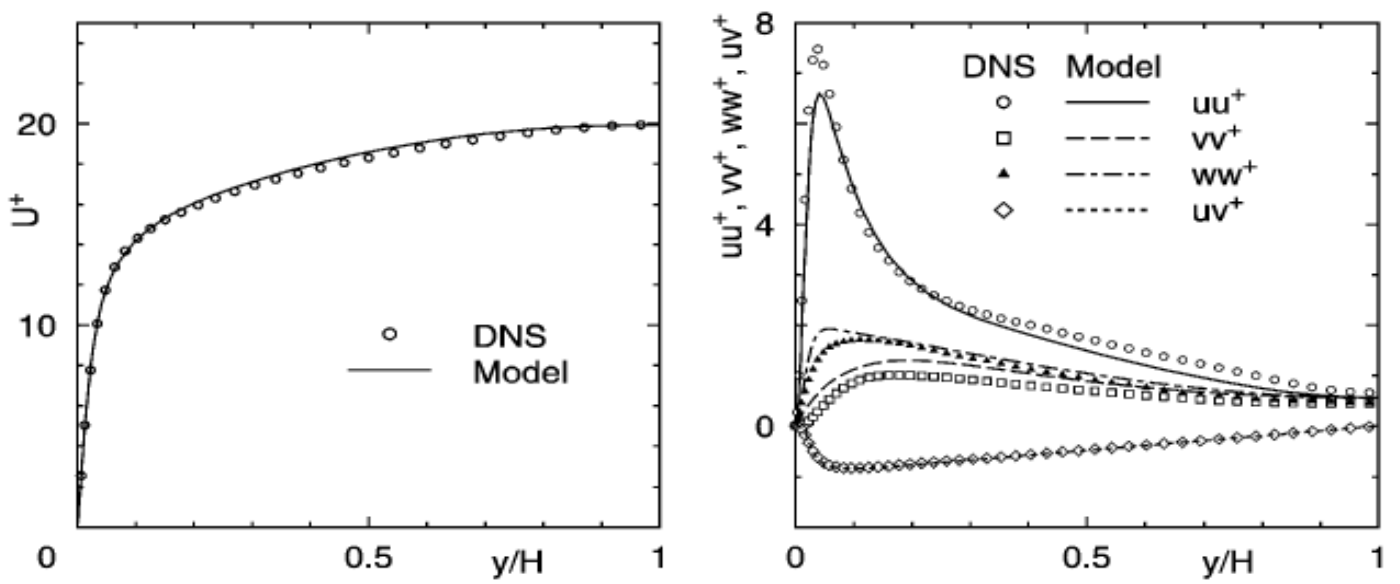

Figure 2.3 - Velocity and Reynolds Components of the Abe et al. (2003) model I for a channel flow of $R e=6875$ Source: Abe et al. (2003) 

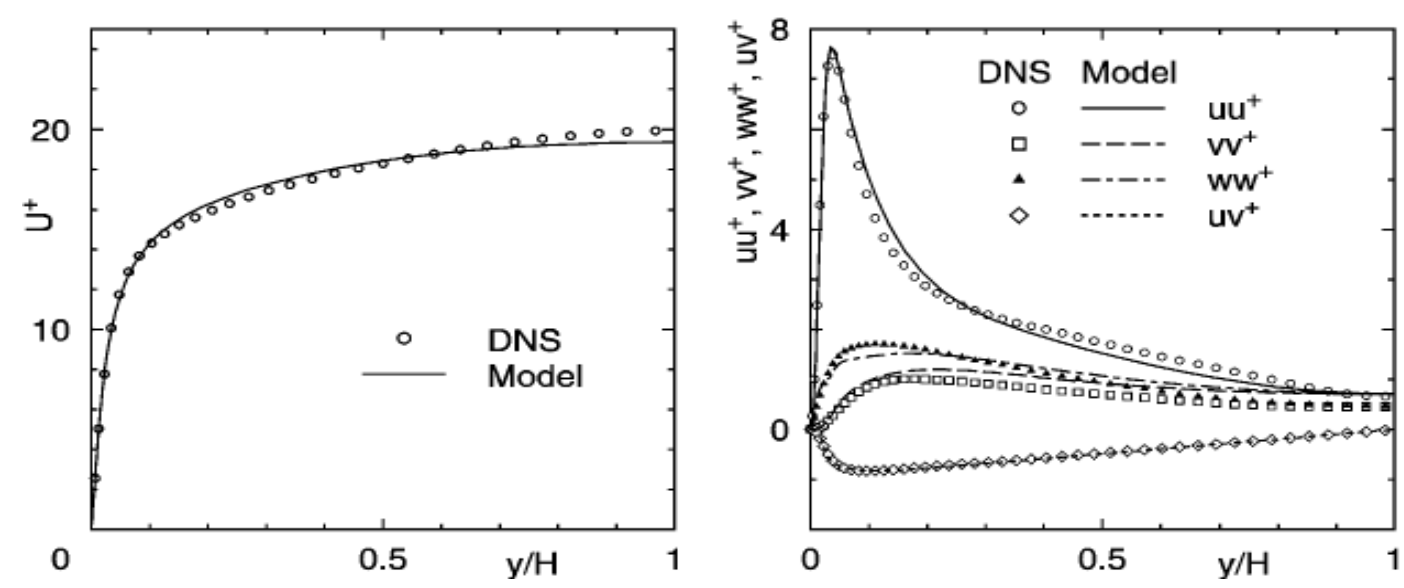

Figure 2. 4 - Velocity and Reynolds Components of the Abe et al. (2003) model III for a channel flow of $R e=6875$ Source: Abe et al. (2003)

Thompson et al. (2010) proposed to quantify Reynolds stress tensor by decomposing it into two orthogonal tensors: the rate of strain and the nonpersistence of straining tensors. The rate of strain is commonly used in non-linear models. The non-persistence of strain is believed to have a deeper physical meaning, for two reasons. First, it incorporates the vorticity tensor, which should have major impact in turbulence. Secondly, one can show that it is frame-invariant, which is not the case with the vorticity tensor used in many non-linear models. The author proposed six models:

- Model I - linear model following the Boussinesq hypothesis.

- Model II - non-linear model written only as a function of the rate of strain.

- Model III - follows the same pattern of model II with the addition of a non-persistence of strain's linear term.

- Model IV- sum of the same linear rate of strain term as model I with the linear non-persistence of strain term.

- Model V - has non-linear terms of the non-persistence of strain and a linear one of the rate of strain

- Model VI - has non-linear terms of both rate of strain and nonpersistence of strain tensors.

Nieckele et al. (2016) performed a priori analysis of the models proposed by Thompson et al. (2010) (figure 2.5) in which DNS data from Thais et al. (2012) was used in order to evaluate the models' predictability. This data was also used to formulate the expressions for damping functions for the nondimensionalized nonlinear terms. 
From Figure 2.5 models III, V and VI are the best models which correctly predicts the Reynolds Stress Tensor. Model I cannot predict any value for any of the components except $a_{x y}$ (which is a characteristic of linear methods). Model II is able to predict all normal tensions but underestimate $a_{x x}$ and $a_{y y}$. Model IV, meanwhile, underestimates $a_{x x}$ and overestimates $a_{y y}$.

The coefficient of the terms proposed by Thompson et al. (2010) models were nondimensionalized following the turbulent kinetic energy and turbulent dissipation rate fields. Alves et al. (2014), however, proposed a new set of nondimensionalization fields by excluding the turbulent dissipation rate and adding the strain tensor intensity. The new set of coefficients' presents a more universal behavior for the Reynolds Numbers tested. Furthermore, the dissipation rate influence, which is obtained by an empirical transport equation, can be reduced.
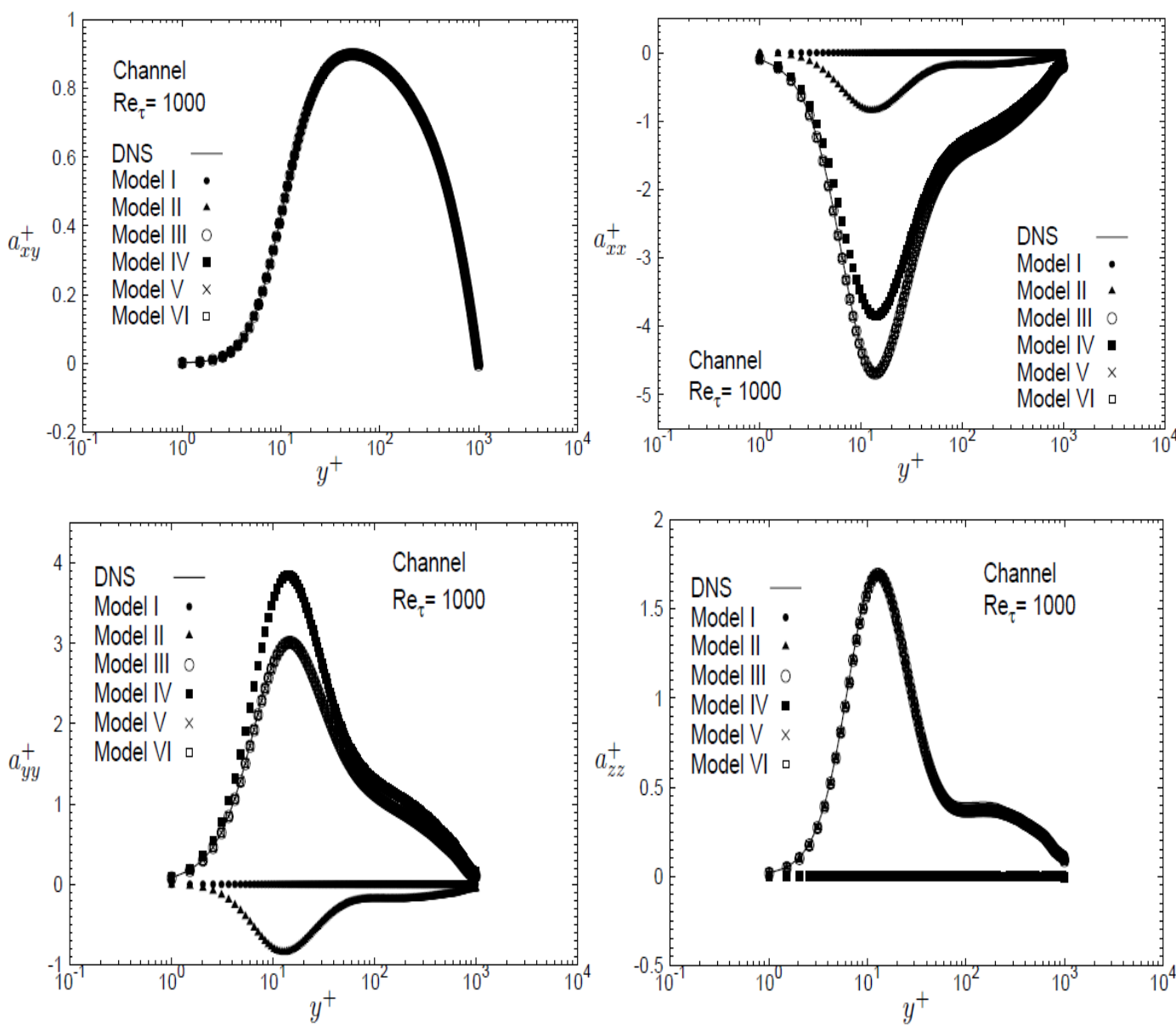

Figure 2.5 - A priori analysis of the six models proposed by (Nieckele et al., 2016) in a Channel Flow with $R e_{\tau}=1000$ 


\section{Mathematical and Numerical Modeling}

In this chapter, the concepts and equations required to develop this work are presented. First, the time average conservation equations are presented, followed by the introduction of the Boussinesq hypothesis. Then, the traditional $\kappa-\varepsilon$ models as well as their version for low Reynolds number are described. Aiming to identify and recommend RANS models with improved prediction capabilities, several nonlinear models are investigated in this work. Here, descriptions of those models are presented in the sequence.

To solve the mathematical model, the open source OpenFoam platform was employed to implement the selected models. The final section of this chapter presents some numerical information. After a brief description of the OpenFoam software, the additional contribution to the software is mentioned. Finally, a few details of numerical parameters selected to the solution of the equations is given.

\subsection{Mathematical Modeling: RANS Methodology}

To determine the flow field, one must solve the mass and momentum equations for a given geometry. Considering a flow of a Newtonian fluid, with constant density and viscosity, the Navier-Stokes equations can be written as

$$
\begin{gathered}
\frac{\partial u_{i}^{*}}{\partial x_{i}}=0 \\
\frac{\partial\left(\rho u_{i}^{*}\right)}{\partial t}+\frac{\partial\left(\rho u_{j}^{*} u_{i}^{*}\right)}{\partial x_{j}}=-\frac{\partial p^{*}}{\partial x_{i}}+\rho g_{i}+\frac{\partial\left(2 \mu D_{i j}^{*}\right.}{\partial x_{j}}
\end{gathered}
$$

where $u_{i}^{*}$ is the velocity component, $p^{*}$ is the pressure, $g_{i}$ the gravity acceleration component, $x_{i}$ is the coordinate system, $\rho$ is the density, $\mu$ is the molecular viscosity and $D_{i j}^{*}$ is the rate of strain.

$$
D_{i j}^{*}=\frac{1}{2}\left(\frac{\partial u_{j}^{*}}{\partial x_{i}}+\frac{\partial u_{i}^{*}}{\partial x_{j}}\right)
$$


The RANS (Reynolds Average Navier Stokes) methodology is based on the concept that all variable can be written as a Reynolds time average term $\bar{\phi}$ and a fluctuation $\phi^{\prime}$ around it, as Eq. (3.4). It should also be mentioned here, that the time average of the product of correlated variables, as the velocity fluctuations, is not zero.

$$
\begin{gathered}
\phi^{*}=\bar{\phi}+\phi^{\prime} \quad ; \bar{\phi}=\frac{1}{\Delta t} \int_{\Delta t} \phi d t \quad ; \quad \overline{\phi^{\prime}}=0 \\
\overline{\phi^{\prime} \varphi^{\prime}} \neq 0
\end{gathered}
$$

The RANS equations can then be obtained, by substituting the velocity field written as the sum of the mean velocity $U_{i}$ with a velocity fluctuation $u_{i}^{\prime}$ :

$$
u_{i}^{*}=U_{i}+u_{i}^{\prime}
$$

Combining the continuity and Navier Stokes equation (3.1 and 3.2), and taking the time average of the resulting expression, the following equation is obtained:

$$
\frac{\partial U_{j} U_{i}}{\partial x_{j}}=-\frac{1}{\rho} \frac{\partial \overline{p^{*}}}{\partial x_{i}}+g_{i}+\frac{2 \mu}{\rho} \frac{\partial D_{i j}}{\partial x_{j}}-\frac{\partial \overline{u_{l}^{\prime} u_{j}^{\prime}}}{\partial x_{j}}
$$

where the new term $\left(-\rho \overline{u_{\imath}^{\prime} u_{\jmath}^{\prime}}\right)$ that appears in the time average expression is called the Reynolds Stress Tensor. Equation (3.7) can also be rewritten as

$$
\frac{\partial U_{j} U_{i}}{\partial x_{j}}=-\frac{\partial p}{\partial x_{i}}+v \frac{\partial^{2} U_{i}}{\partial x_{j}^{2}}+\frac{\partial a_{i j}}{\partial x_{j}}
$$

where $v=\mu \backslash \rho$ is the kinematic viscosity; $a_{i j}$ is the deviatoric part of the Reynolds stress tensor

$$
\begin{gathered}
a_{i j}=-\overline{u_{\imath}^{\prime} u_{\jmath}^{\prime}}+\frac{2}{3} \kappa \delta_{i j} \\
\kappa=\frac{1}{2} \overline{u_{k}^{\prime} u_{k}^{\prime}}
\end{gathered}
$$

and $\kappa$ as the turbulence kinetic energy. $p$ is a modified pressure term that incorporates the gravitational term and the turbulent dynamic pressure, defined as: 


$$
p=\frac{\overline{p^{*}}}{\rho}-g x_{i} \boldsymbol{e}_{\boldsymbol{i}} \cdot \boldsymbol{e}_{z}+\frac{2}{3} \kappa
$$

The equations can be nondimensionalized with a characteristic dimension $H$ and with the friction velocity $u_{\tau}$, defined as

$$
u_{\tau}=\sqrt{\frac{\tau_{w}}{\rho}}
$$

where $\tau_{w}$ is the shear tension at the wall. The dimensionless variables are

$$
U_{i}^{+}=\frac{U_{i}}{u_{\tau}} \quad ; \quad x_{i}^{+}=\frac{\rho u_{\tau} x_{i}}{\mu} ; \quad P^{+}=\frac{p}{u_{\tau}^{2}} \quad ; \quad a_{i j}^{+}=\frac{a_{i j}}{u_{\tau}^{2}}
$$

Resulting in the following conservation equations are

$$
\frac{\partial U_{j}^{+}}{\partial x_{j}^{+}}=0 \quad ; \quad \frac{\partial U_{j}^{+} U_{i}^{+}}{\partial x_{j}^{+}}=-\frac{\partial p^{+}}{\partial x_{i}^{+}}+\frac{1}{R e_{\tau}} \frac{\partial^{2} U_{i}^{+}}{\partial x_{j}^{+2}}+\frac{\partial a_{i j}^{+}}{\partial x_{j}^{+}}
$$

Indicating that the flow field is greatly influenced by the Reynolds number $R e_{\tau}$

$$
R e_{\tau}=\frac{\rho u_{\tau} H}{\mu}
$$

Since the Reynolds stress tensor is unknown, this model still needs closure expressions. Hence, different models, that were investigated in the present work to determine it, will be discussed in the next subsection.

\subsection{The Boussinesq Hypothesis}

For a Newtonian fluid, in the laminar regime, the shear tensor is linearly proportional to twice the rate of strain tensor $\left(2 D_{i j}\right)$. Further, it is observed that turbulence increases the flow's diffusion. Therefore, Boussineq postulated that the Reynolds Tensor should have the same behavior as the shear tension for a Newtonian fluid (Pope, 2000). Hence, it could be modelled analogously as:

$$
\rho a_{i j}=\mu_{t} 2 D_{i j}
$$


where $\mu_{t}$ is the turbulent viscosity, $D_{i j}$ is the rate of strain of the time average flow. This property, however is not attributed to the fluid as in molecular viscosity $\mu$. It is a characteristic of the flow.

Even with the additional relation, Eq. (3.16), to determine the flow field, closure expressions are still required since the turbulent viscosity is not defined. There are many models to predict the turbulent viscosity, and the majority is based on the assumption that it can be determined in an analogous form as the molecular viscosity, i.e., based on a characteristic velocity $V_{c}$ and characteristic length $l_{c}$ of an eddy.

$$
\mu_{t} \sim \rho V_{c} l_{c}
$$

The several RANS models differ in the identification of these characteristics terms. The characteristic velocity is often considered as being proportional to the turbulent kinetic energy.

$$
V_{c}=\sqrt{\kappa}
$$

This seems to be a good choice and it is employed in a vast number of models. The selection of the characteristic length is not straight forward, therefore it is more difficult to define, and it is the mayor difference between the models.

One of the most popular models in the academia and industry is the $\kappa-\varepsilon$ model. Therefore, it was selected to be investigated in the present work.

\subsubsection{The $\kappa-\varepsilon$ Model}

The characteristic length of a $\kappa-\varepsilon$ models is based on the rate of dissipation of the turbulent kinetic energy $\varepsilon$ (specific power dissipated by an eddy)

$$
\varepsilon=\frac{F V_{c}}{\rho l_{c}^{3}} \approx \frac{C_{d} \rho V_{c}^{2} l_{c}^{2} V_{c}}{\rho l_{c}^{3}} \approx \frac{\kappa^{3 / 2}}{l_{c}}
$$

In the previous equation $F$ is the drag force and $C_{d}$ is a drag coefficient.

As a result, the turbulent viscosity can be obtained by

$$
\mu_{t}=\frac{C_{\mu} \rho \kappa^{2}}{\varepsilon}
$$


where $C_{\mu}$ is an empirical constant typically equal to 0.09 . The turbulent viscosity is, therefore, expressed as a function of $\kappa$ and $\varepsilon$ though these two entities still requires to be modeled. The derivation of the standard $\kappa-\varepsilon$ model's $\kappa$ and $\varepsilon$ equations can be found in (Pope, 2000) and they have the following form for a permanent incompressible flow:

$$
\begin{gathered}
\frac{\partial\left(\rho \kappa U_{j}\right)}{\partial x_{j}}=\frac{\partial}{\partial x_{j}}\left[\left(\mu+\frac{\mu_{t}}{\sigma_{\kappa}}\right) \frac{\partial \kappa}{\partial x_{j}}\right]+G_{\kappa}-\rho \varepsilon \\
\frac{\partial\left(\rho \varepsilon U_{j}\right)}{\partial x_{j}}=\frac{\partial}{\partial x_{j}}\left[\left(\mu+\frac{\mu_{t}}{\sigma_{\varepsilon}}\right) \frac{\partial \varepsilon}{\partial x_{j}}\right]+C_{\varepsilon 1} G_{\kappa} \frac{\varepsilon}{\kappa}-C_{\varepsilon 2} \rho \frac{\varepsilon^{2}}{\kappa}
\end{gathered}
$$

where $\sigma_{\kappa}, \sigma_{\varepsilon}, C_{\varepsilon 1}$ and $C_{\varepsilon 2}$ are empirical constants equal to $1,1.3,1.44$ and 1.93 respectively. $G_{\kappa}$ is the production of turbulent kinetic energy and it is given by

$$
G_{\kappa}=-\rho \overline{u_{\imath}^{\prime} u_{\jmath}^{\prime}} \frac{\partial U_{i}}{\partial x_{j}}
$$

By neglecting compressibility effects, it can be written as

$$
G_{\kappa}=\rho a_{i j} \frac{\partial U_{i}}{\partial x_{j}}
$$

The $\kappa-\varepsilon$ equations must only be applied in regions far from the wall. Near the wall, different viscous regions can be found, as it is illustrated in Fig. 3.1. To correctly predict the Reynolds Tensor inside this region, laws of wall (Bradshaw \& Huang, 1995) must be used instead of the main equations.

Depending on the Reynolds number $R e_{\tau}$, for the same dimensional distance to the wall, the resulting dimensionless wall distance $y^{+}$is different and distinct near wall treatment must be applied.

The standard wall law, is based on the log-wall. Therefore, it is valid for $y^{+}>30$, however, frequently the buffer layer is neglected and the standard law is applied for $y^{+}>11.5$. In this case, the following expressions are composed

$$
u^{+}=\frac{1}{k} \ln y^{+}+5.5
$$

where $u^{+}$is the velocity component parallel to the wall, and $k \approx 0.4$ is the von Kármán constant (Pope, 2000). To guarantee mass conservation the gradient of the 
normal velocity component is neglected, i.e., $\partial u_{n} / \partial x_{n}=0$. The boundary condition for $\kappa$ and $\varepsilon$ are defined considering that there is equilibrium between production and destruction of $\kappa$, resulting in

$$
\frac{\partial \kappa}{\partial n}=0 \quad \text { since } \frac{\tau_{w}}{\rho}=C_{\mu}^{\frac{1}{2}} \kappa \approx \text { constant } \quad \text { and } \quad \varepsilon=\frac{C_{\mu}^{3 / 4} \kappa^{3 / 2}}{k y}
$$

To model the viscous sublayer, $y^{+}<5$, one option is to use a variant of the $\kappa-\varepsilon$ model called low Reynolds $\kappa-\varepsilon$ Model which will be explained in the next subsection.

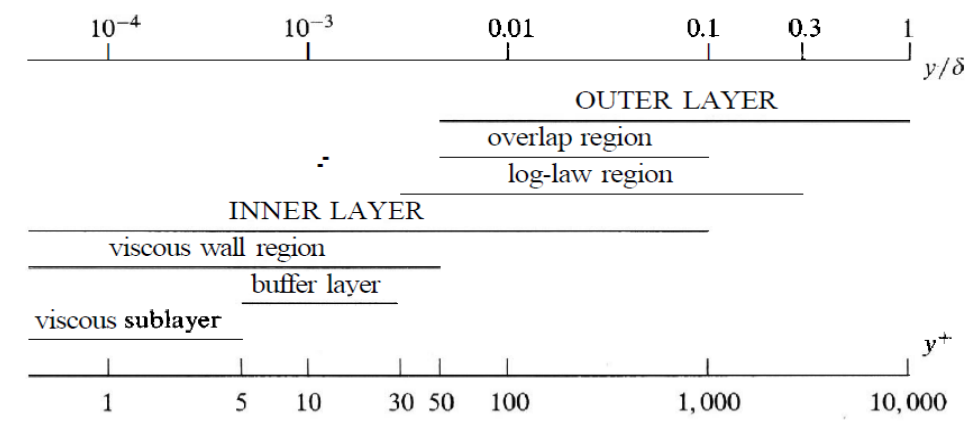

\begin{tabular}{lcl}
\hline Region & Location & Defining property \\
\hline \hline Inner layer & $y / \delta<0.1$ & $\begin{array}{l}\text { (U) determined by } \boldsymbol{u}_{\tau} \text { and } y^{+} \text {, indepen- } \\
\text { dent of } U_{0} \text { and } 6\end{array}$ \\
Viscous wall region & $y^{+}<50$ & $\begin{array}{l}\text { The viscous contribution to the shear } \\
\text { stress is significant } \\
\text { The Reynolds shear stress is negligible } \\
\text { compared with the viscous stress } \\
\text { Viscous sublayer }\end{array} y^{+}<5$ \\
Outer layer & $y^{+}>50$ & $\begin{array}{l}\text { Direct effects of viscosity on (U) are } \\
\text { negligible } \\
\text { Region of overlap between inner and } \\
\text { outer layers (at large Reynolds numbers) } \\
\text { The log-law holds } \\
\text { The region between the viscous sublayer } \\
\text { and the log-law region }\end{array}$ \\
\hline \hline
\end{tabular}

Figure 3.1 - Near wall classification regions. Adapted from (Pope, 2000)

\subsubsection{Linear Low Reynolds K- $\varepsilon$ Models}

The Low Reynolds $\kappa-\varepsilon$ Models (Wilcox, 2006) do not apply wall functions near the walls. Instead, a damping function $f_{\mu}$ is applied at near wall regions to evaluate the turbulent viscosity.

$$
\mu_{t}=\frac{f_{\mu} C_{\mu} \rho \kappa^{2}}{\tilde{\varepsilon}}
$$

At the wall, considering non-slip condition, all velocity components are null, as well as the turbulent Kinect energy $\left(\kappa_{w}=0\right)$. To evaluate the dissipation value at 
the wall, asymptotic analyses can be carried out and different expressions can be employed, such as $\varepsilon_{w}=2 \mu(\partial \sqrt{\kappa} / \partial y)^{2}$ (Wilcox, 2006).

The dissipation can be rewritten relative to its value at the wall, aiming to improve or facilitate the definition of dissipation boundary condition so that $\tilde{\varepsilon}=0$ at the wall.

$$
\varepsilon=\tilde{\varepsilon}-E_{0}
$$

Damping functions $f_{1}$ and $f_{2}$ are also introduced in the dissipation equation to damp the production and destruction of $\varepsilon$ at the near wall region. The resulting equation can be written as

$$
\frac{\partial\left(\rho \tilde{\varepsilon} U_{j}\right)}{\partial x_{j}}=\frac{\partial}{\partial x_{j}}\left[\left(\mu+\frac{\mu_{t}}{\sigma_{\varepsilon}}\right) \frac{\partial \tilde{\varepsilon}}{\partial x_{j}}\right]=f_{1} C_{\varepsilon 1} G_{\kappa} \frac{\tilde{\varepsilon}}{\kappa}-f_{2} C_{\varepsilon 2} \rho \frac{\tilde{\varepsilon}^{2}}{\kappa}+E
$$

These damping functions $\left(f_{\mu}, f_{1}\right.$ and $\left.f_{2}\right)$ and the parameters $E$ and $E_{0}$ vary depending on the chosen model, while the $\kappa$ equation stays the same as Eq. (3.21).

Table 3.1 shows the damping functions, parameters and constants of four linear Low-Reynolds Models selected to be investigated in this work: The Lam Bremhorst (Lam \& Bremhorst, 1981), Launder Sharma (Launder et al., 1977), Lien Leschziner (Lien \& Leschziner, 1993) and Modified Rodi Mansour (Michelassi et al. 1993) models.

\subsection{Non-Linear Models}

Non-Linear $\kappa-\varepsilon$ models extend the basis of the Boussinesq hypothesis Eq. (3.16) with non-linear terms, as:

$$
\rho a_{i j}=\mu_{t} 2 D_{i j}+N L S_{i j}
$$

where $N L S_{i j}$ represents the non-linear stress tensor whose definition differ from each non-linear model.

At the present work, the Lien Cubic model Lien et al. (1991) was selected to be investigated. However, the main focus of the present work is to evaluate the family of non-linear models, developed based on the orthogonal decomposition by Thompson (2008). An analysis a priori of the performance of these models for a channel and boundary layer flow, was presented by Nieckele et al. (2016) 
Table 3.1 - Low Reynolds damping functions, parameters and constants

\begin{tabular}{|c|c|c|c|c|}
\hline Model & Lam BremHorst & Launder Sharma & Lien Leschziner & Modified Rodi Mansour \\
\hline$f_{\mu}$ & $\left(1+\frac{20.5}{R_{t}}\right)\left(1+e^{-0.165 R_{y}}\right)^{2}$ & $1 / \exp \left(\frac{3.4}{1+\frac{R_{t}}{50}}\right)$ & $\frac{1+e^{-A_{\mu} y^{*}}}{1+e^{-A_{\varepsilon} y^{*}}}$ & $1-e^{-2 \times 10^{-4} y^{+}-6 \times 10^{-4} y^{+^{2}}+2.5 \times 10^{-7} y^{+^{3}}}$ \\
\hline$f_{1}$ & $1+\left(\frac{0.05}{f_{\mu}}\right)^{3}$ & 1 & 1 & 1 \\
\hline$f_{2}$ & $1-1 / \exp \sqrt{R_{t}}$ & $1-0.3 e^{-\min \left(50,\left(R_{t}\right)^{2}\right)}$ & $1-0.3 / \exp \sqrt{R_{t}}$ & $\begin{array}{c}f_{2}^{2} f_{2}^{1}+f_{3}-1 \\
f_{2}^{2}=1-0.22 e^{-0.3357 \sqrt{R_{t}}} \\
f_{2}^{1}=\frac{\tilde{\varepsilon}}{\varepsilon} \quad ; \quad f_{3}=e^{1.8\left(R_{p}\right)^{3}}\end{array}$ \\
\hline$E$ & 0 & $2 \mu \mu_{t}\left(\left|\nabla^{2} \bar{U}\right|\right)^{2}$ & $C_{\varepsilon 2}\left(C_{\mu}\right)^{0.75} \frac{f_{2} \varepsilon \sqrt{\kappa}}{l_{e}} e^{-A_{\varepsilon} \sqrt{y^{*}}}$ & $1.2 v v_{t}\left(\frac{\partial^{2} \bar{U}}{\partial y^{2}}\right)^{2}+\frac{0.0075 v \kappa}{\varepsilon} \frac{\partial \kappa}{\partial y} \frac{\partial \bar{U}}{\partial y} \frac{\partial^{2} \bar{U}}{\partial y^{2}}$ \\
\hline$E_{0}$ & 0 & $2 \mu(|\nabla \sqrt{\kappa}|)^{2}$ & 0 & $\varepsilon e^{-0.095 R_{y}}$ \\
\hline Constants & $\begin{array}{c}C_{\mu}: 0.09 ; C_{\varepsilon 1}: 1.44 \\
C_{\varepsilon 2}: 1.92 ; \sigma_{\kappa}: 1.0 ; \sigma_{\varepsilon}: 1.3\end{array}$ & $\begin{array}{c}C_{\mu}: 0.09 ; C_{\varepsilon 1}: 1.44 ; \\
C_{\varepsilon 2}: 1.92 ; \sigma_{\kappa}: 1.0 ; \sigma_{\varepsilon}: 1.3\end{array}$ & $\begin{array}{c}C_{\mu}: 0.09 ; C_{\varepsilon 1}: 1.44 ; C_{\varepsilon 2}: 1.92 \\
A_{\mu}: 0.016 ; \quad A_{\varepsilon}: 0.0022 \\
\sigma_{\kappa}: 1.0 ; \quad \sigma_{\varepsilon}: 1.3\end{array}$ & $\begin{array}{c}C_{\mu}: 0.09 ; C_{\varepsilon 1}: 1.44 ; C_{\varepsilon 2}: 1.92 \\
\sigma_{\kappa}: 1.3 ; \sigma_{\varepsilon}: 1.3\end{array}$ \\
\hline
\end{tabular}




\subsubsection{The K- $\varepsilon$ Lien Cubic Model}

The Lien Cubic model (Lien et al., 1991) is a Non-Linear Low Reynolds model which combines the extended Boussinesq basis Eq. (3.30) with Eq. (3.21) and Eq. (3.29). This model was already implemented in numerical platforms such as OpenFOAM. For this model the non- linear terms, constants and parameters are specified in Table 3.2 .

Table 3.2 - Lien Cubic Parameters and Constants

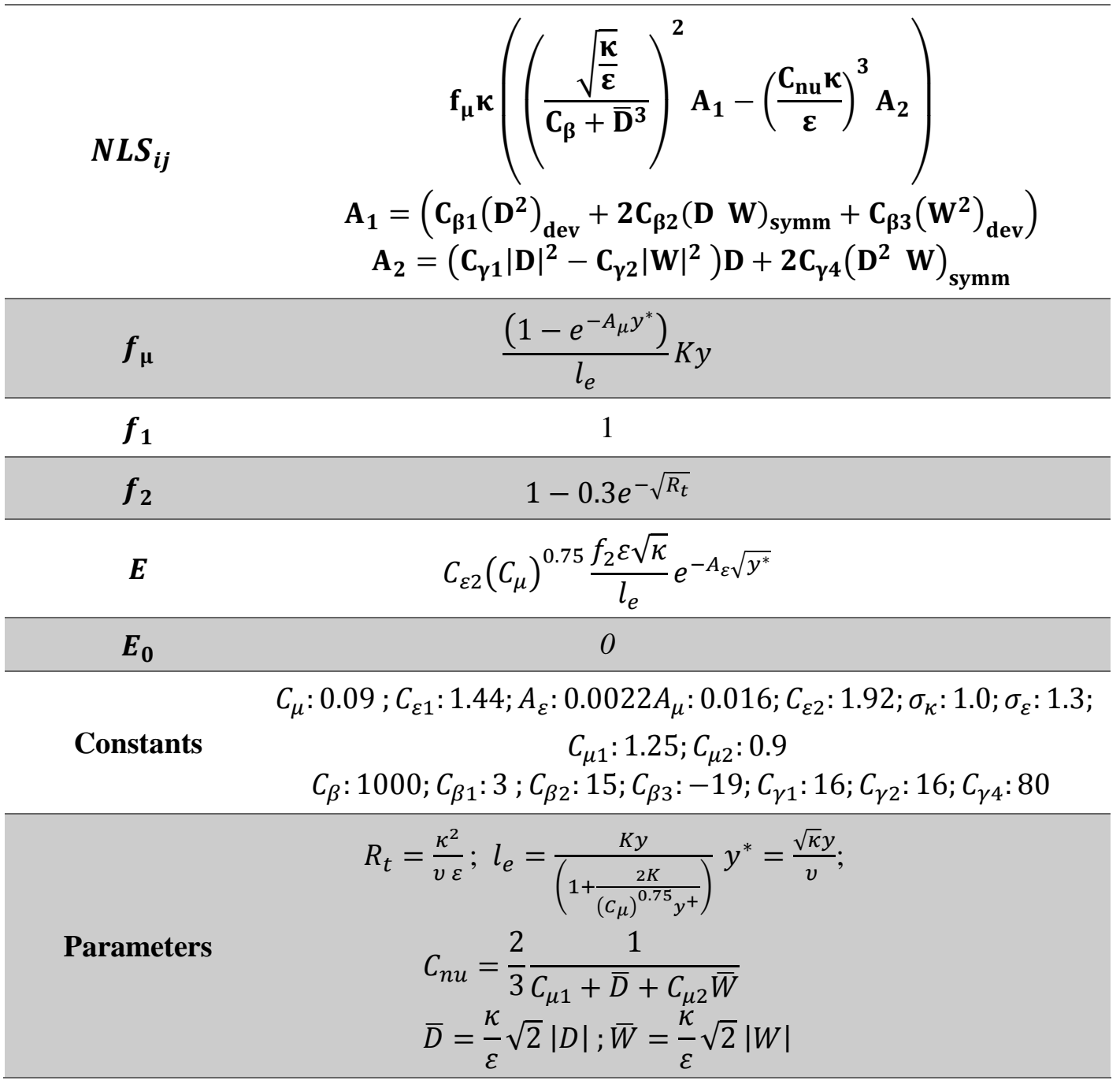

\subsubsection{Non-Linear Models Based on Orthogonal Decomposition}

The non-linear models selected to be investigated here, were developed based on the mathematical concept of tensor decomposition in an orthogonal tensor basis first introduced by Thompson, (2008) and adapted into a turbulence model by Thompson et al., (2010). There are two types of orthogonal decomposition. In the 
first one, the tensor $A_{i j}$ is written as the sum of a linear component $\alpha B_{i j}$ plus an orthogonal component $B_{i j}^{\perp}$, hence:

$$
A_{i j}=\alpha B_{i j}+B_{i j}^{\perp}
$$

In the second decomposition, the tensor is divided into in-phase and out-phase parts. The in-phase part is composed of a tensor $B_{i j}{ }_{A}^{H}(\mathrm{H}$ denotes that the term is written in the base of $H_{i j}$ eigenvalues) that has the same eigenvectors as $A_{i j}$. Because of that, the $B_{i j_{A}}{ }^{H}$ component is able to sweep a broader tensor field than the linear term of the first decomposition $\left(\alpha B_{i j}\right)$. However, the out-phase $\widetilde{B}_{i j_{A}}{ }_{A}^{H}$ has different eigenvectors than $A_{i j}$, therefore:

$$
A_{i j}=B_{i j_{A}}^{H}+\tilde{B}_{i j_{A}}{ }^{H}
$$

Using Cayley - Hamilton theorem, the in-phase term can be rewritten as:

$$
B_{i j_{A}}^{H}=\alpha_{0} I+\alpha_{h} H+\alpha_{h 2} H^{2}
$$

Thompson et al. (2010) proposed a family of turbulent model, based on projecting the Reynolds stress tensor into different sets of tensor basis based on mean kinematic quantities: the rate of strain tensor $D_{i j}$ (Eq. 3.3) and the nonpersistence of straining tensor $P_{i j}$

$$
P_{i j}=D_{i j} W_{i j}^{*}-W_{i j}^{*} D_{i j}
$$

where $W^{*}$ is the relative rate of rotation defined as $W_{i j}^{*}=W_{i j}-\Omega_{i j}^{D}, W$ is the skew symmetric of the velocity gradient

$$
W_{i j}=\frac{1}{2}\left(\frac{\partial U_{j}}{\partial x_{i}}-\frac{\partial U_{i}}{\partial x_{j}}\right)
$$

$\Omega^{D}$ is the rate of rotation tensor of the eigenvalues of the flow $\left(\Omega^{D}=\dot{e}_{k}^{D} e_{k}^{D}\right), e_{k}^{D}$ is the unit eigenvector of $D_{i j}$ and $\dot{e}_{k}^{D}$ is its material derivation.

Six models were evaluated by Nieckele et al. (2016) employing a priori analysis by comparing the models' prediction with experimental and DNS data for 
boundary layer and channel flow. From this set, four models were selected to be analyzed here through a posterior analysis:

$$
\begin{gathered}
M_{I}: \quad a_{i j}=a_{D 1} 2 D_{i j} \\
M_{I I}: \quad a_{i j}=a_{D 0} I+a_{D 1} 2 D_{i j}+a_{D 2} D_{i j}^{2} \\
M_{I I I}: \quad a_{i j}=a_{D 0} I+a_{D 1} 2 D_{i j}+a_{D 2} D_{\mathrm{ij}}^{2}+a_{\beta} P_{i j} \\
M_{I V}: \quad a_{i j}=a_{D 1} 2 D_{i j}+a_{\beta} P_{i j}
\end{gathered}
$$

Model I is a linear model, with the Reynolds Tensor directly proportional to $D_{i j}$. Model II is an in-phase/out-phase decomposition of the same tensor, therefore, the error associated to it is, by definition, lower than model I. In Model III the same in-phase/out-phase decomposition occurs, but now with the orthogonal tensor being modeled by a linear decomposition using $P_{i j}$, hence, its error is inferior than model II and IV. Finally, Model IV is the same linear decomposition as model I and the orthogonal tensor is modeled by another linear decomposition, moreover, its error is lower than model I but higher than model III.

The coefficients $a_{D i}$ and $a_{\beta i}$ were determined by Nieckele et al. (2016) employing noble (experimental and DNS) data. Aiming to propose a general model these coefficients should be nondimensionalized by the field data.

\subsubsection{Non-linear $\kappa-\varepsilon$ Models}

Inspired by the popular $\kappa-\varepsilon$ models, Nieckele et al. (2016) nondimensionalized the coefficients $a_{D 0 i}, a_{D 1 i}, a_{D 2 i}$ and $a_{\beta i}$ using $\kappa$ and $\varepsilon$ parameters.

The coefficient $a_{D 1}$ is the turbulent viscosity, and it is nondimensionalized employing a damping function $f_{\mu}$, similarly to existing Low-Reynolds $\kappa-\varepsilon$ models

$$
a_{D 1}=f_{\mu} C_{\mu} \frac{\kappa^{2}}{\varepsilon}
$$

After performing a comparison of several available damping function $f_{\mu}$ expressions with the DNS data, Nieckele et al. (2016) recommended the modified 
Rodi and Mansur damping function $f_{\mu}$ (Michelassi et al. 1993), showed in Section 3.2.2, and repeated here

$$
f_{\mu}=1.0-\exp \left(3 .-0.0002 y^{+}-0.00065 y^{+2}\right)
$$

It should be mentioned here that, to apply Rodi and Mansur damping function, it is recommended to also consider their recommendation of damping coefficients $f_{1}$ and $f_{2}$ in the dissipation conservation equation, as shown in Table 3.1.

The coefficients of the nonlinear $D_{i j}^{2}$ and $P_{i j}$ terms were nondimensionalized with $\kappa^{3} / \varepsilon^{2}$ and employed damping functions

$$
a_{D 2}=f_{\mu 2} C_{\mu 2} \frac{\kappa^{3}}{\varepsilon^{2}} \quad ; \quad a_{\beta}=f_{\beta} C_{\beta} \frac{\kappa^{3}}{\varepsilon^{2}}
$$

The coefficient $a_{D 0}$ is determined from $a_{D 2}$ coefficient in order to guarantee that the tensor $a_{i j}$ is traceless. Further, it is nondimensionalized with the turbulent kinetic energy

$$
a_{D 0}=\kappa C_{D 0} \quad ; \quad a_{D 0}=-\frac{2}{3} a_{D 2} \dot{\gamma}^{2}
$$

where $\dot{\gamma}^{2}$ is the trace of $D_{i j}^{2}$, and $\dot{\gamma}$ is the intensity of the rate of strain tensor

$$
\dot{\gamma}=\sqrt{\frac{1}{2} D_{i j} D_{j i}}
$$

Nieckele et al. (2016) verified that the parameter $C_{\mu}$ presented a small dependence on Reynolds number and they recommend to define $C_{\mu}=0.07$, while the recommended value of Rodi \& Mansur is 0.09. Here, an investigation of the impact of the $C_{\mu}$ parameter in the flow field was conducted for $R e_{\tau}=1000$, and it is shown in Appendix A.1. Based on the tests performed, at the present work, the variable was set as $C_{\mu}=0.075$.

Nieckele et al. (2016) defined the others two parameters $C_{\mu 2}$ and $C_{\beta}$ as -0.05 and 0.055 respectively. They also proposed expression for the damping functions $f_{\mu 2}$ and $f_{\beta}$. However, at the present work, after investigating the performance of the models with the damping recommended by the reference, a better adjustment of them with the DNS data was performed and the derivation is presented at Appendix A.2. The resulting functions are 


$$
\begin{gathered}
f_{\mu 2}=\frac{\tanh \left(6.15 \times 10^{-4}\left(y^{+}\right)^{2.135}\right)}{\tanh \left(10^{-5}\left(y^{+}\right)^{5.8}\right)}+6.1 \times 10^{-2} e^{\left(-\frac{y^{+}}{300}\right)^{2}} \\
f_{\beta}=\frac{\tanh \left(2.56 * 10^{-4}\left(y^{+}\right)^{2.305}\right)}{\tanh \left(1.3 * 10^{-5}\left(y^{+}\right)^{6.5}\right)}+1.818 * 10^{-2} e^{\left(-\frac{y^{+}}{300}\right)^{2}}+\frac{60.6}{\left(y^{+}\right)^{4}}+0.02727
\end{gathered}
$$

\subsubsection{Non-linear $\kappa-\dot{\gamma}$ Models}

Since the greatest limitation of $\kappa-\varepsilon$ models is the prediction of $\varepsilon$, Alves et al. (2014) have proposed to nondimensionalize these coefficients employing the turbulent kinetic energy $\kappa$, and the intensity of the rate of strain tensor $\dot{\gamma}$. This would reduce the influence of the $\varepsilon$ field in the overall model, however, it is still necessary to be calculate the destruction term in the kinetic energy equation. Thus, the $\varepsilon$ equation is still not fully eliminated from the model. The proposed nondimensionalization is:

$$
\begin{gathered}
a_{D 1}=C_{\mu}^{\gamma} \frac{\kappa}{\dot{\gamma}} \\
a_{D 2}=C_{\mu 2}^{\gamma} \frac{\kappa}{\dot{\gamma}^{2}} \quad ; \quad a_{\beta}=C_{\beta}^{\gamma} \frac{\kappa}{\dot{\gamma}^{2}} \\
a_{D 0}=\kappa C_{D 0}^{\gamma} \quad ; \quad a_{D 0}=-\frac{2}{3} a_{D 2} \dot{\gamma}^{2}
\end{gathered}
$$

In a similar way as employed in subsection 3.3.3, and as most Low Reynolds methods, the coefficients $C_{\mu}^{\gamma}, C_{\mu 2}^{\gamma}$ and $C_{\beta}^{\gamma}$ were calibrated using DNS data, (Appendix B). The functions which best describe $C_{\mu}^{\gamma}$ are

$$
\begin{gathered}
C_{\mu-\gamma}=0.0054 y^{+} ; y^{+}<3 \\
C_{\mu-\gamma}=\frac{-0.007+0.0086 y^{+}}{1+0.056 y^{+}+1.5 \times 10^{-5}\left(y^{+}\right)^{2}} ; 3<y^{+} \leq \hat{y}^{+} \\
C_{\mu-\gamma}=5.6 \times 10^{-10}\left(y_{e f f}^{+}\right)^{3}+9 \times 10^{-8}\left(y_{e f f}^{+}\right)^{2}+C_{\mu}^{\gamma}\left(y_{e f f}^{+}\right) ; \hat{y}^{+}<y^{+}
\end{gathered}
$$

where $y_{e f f}^{+}$is written as the following difference $y_{e f f}^{+}=y^{+}-\hat{y}^{+}$and $\hat{y}^{+}$is the $y^{+}$value of maximum $C_{\mu-\gamma}$ which can be defined as a function of the Reynolds Number: $\hat{y}^{+}=0.2734 R e+44.745$.

The coefficient of $C_{\mu 2}^{\gamma}$ of the non-linear term $D_{i j}^{2}$ is 


$$
\begin{gathered}
C_{\mu 2-\gamma}=0.2277 ; y^{+}<1 \\
C_{\mu 2-\gamma}=0.5622\left(y_{\text {log }}^{+}\right)^{4}-2.0685\left(y_{\text {log }}^{+}\right)^{3}+1.948\left(y_{\text {log }}^{+}\right)^{2} \\
-0.1167\left(y_{\text {log }}^{+}\right)+0.2277 ; 1<y^{+} \leq 69 \\
C_{\mu 2-\gamma}=C_{\mu 2-\gamma}\left(y^{+}=69\right) ; 69<y^{+}
\end{gathered}
$$

where $y_{\log }^{+}=\log _{10}\left(y^{+}\right)$.

Finally the coefficient $C_{\beta}^{\gamma}$ is

$$
\begin{gathered}
C_{\beta-\gamma}=0.3716 ; y^{+}<1 \\
C_{\beta-\gamma}=0.04835\left(y_{\text {log }}^{+}\right)^{5}-0.0859\left(y_{\text {log }}^{+}\right)^{4}-0.1759\left(y_{\text {log }}^{+}\right)^{3}+0.2784\left(y_{\text {log }}^{+}\right)^{2} \\
-0.0269\left(y_{\text {log }}^{+}\right)+0.3716 ; 1<y^{+} \leq 95 \\
C_{\beta-\gamma}=2.38 \times 10^{-10}\left(y^{+}\right)^{3}+2.38 \times 10^{-7}\left(y^{+}\right)^{2}+1.117 \times 10^{-4} y^{+} \\
+0.1993 ; 95<y^{+}
\end{gathered}
$$

\subsection{Numerical Model}

As already mentioned the OpenFoam software was chosen to implement the turbulent models selected and to simulate the flow. It is an open source platform based on the finite volume methodology (Patankar, 1980) to solve many continuum phenomena problems, such as turbulence. OpenFoam is written in $\mathrm{C}++$ computational language and can be downloaded for Linux or windows for free in (openFOAM, 2018) (used version: 4.1).

The non-linear models developed by Thompson (2008) and the Modified Rodi-Mansur Michelassi et al. (1993) were not available in the software, so they were implemented in the platform. However, the following models were already implemented in the version 4.1 of the code OpenFoam: Lam-BremHorst, LaunderSharma and Lien_Leschziner, as well as the non-linear model $\kappa-\varepsilon$ Lien Cubic Model.

Before presenting a few numerical details employed in the discretization of the conservation equation, the general conservation equation is shown below. It represents a net convective-diffusive flux equal to a source term. 


$$
\nabla \cdot(\rho \boldsymbol{U} \Phi)-\nabla \cdot(\Gamma \nabla \Phi)=S_{c}+S_{p} \Phi
$$

The Finite Volume method consists of dividing the domain of interest in control volumes and integrating the conservation equation in each control volume, resulting on a global conservation equation. To integrate the conservation equations in the volume, OpenFOAM employs the Gauss divergence theorem, transforming the volume integral into a surface integral. The convective flux and diffusive flux terms become

$$
\begin{gathered}
\int_{\forall_{P}} \nabla \cdot(\rho \boldsymbol{U} \Phi) d \forall=\oint_{\partial \forall_{P}} \boldsymbol{n} \cdot(\rho \boldsymbol{U} \Phi) d A=\sum_{f} n_{f} \cdot(\rho \boldsymbol{U} \Phi)_{f} A_{f} \\
\int_{\forall_{P}} \nabla \cdot(\Gamma \nabla \Phi) d \forall=\oint_{\partial \forall_{P}} \Gamma \boldsymbol{n} \cdot \nabla \Phi d A=\sum_{f} \Gamma_{f} n_{f} \cdot(\nabla \Phi)_{f} A_{f}
\end{gathered}
$$

OpenFOAM employs co-located variables and the convective flux of the momentum equation at the control volume faces $f$ were approximated with the upwind scheme with weight factors based on the local gradient, while for the $\kappa$ and $\varepsilon$, linear scheme with limiter factor was employed. The diffusive flux were determined based on linear approximation to determine the gradient at the control volume faces.

As recommended by (Patankar, 1980), the source term of each conservation equation was linearized in such a way as to guarantee $S_{p} \leq 0$. Table 3.3 presents for each variable $\Phi$ solved, the corresponding diffusion coefficient $\Gamma$ and source term $\left(S_{c}\right.$ and $\left.S_{P}\right)$. The new contribution for the software are the non-linear contribution of the Reynolds stress tensor, which were treated explicitly. In the present work, the additional source term $E$ of $\varepsilon$ conservation equation was also added to the software. In Table 3.3, the contribution for all non-linear models is considered, with $a_{\beta}=0$ for Model II and $a_{D 2}=0$ for Model IV.

The velocity and pressure coupling was solved by the SIMPLE (Semi implicit method of Pressure linked equation) (Patankar, 1980) algorithm.

To solve the system of equations the GAMG (Geometric Algebraic MultiGrid) algorithm was set. 
Sub relaxation factors were applied to all transport equations. Very small values were applied at the beginning of the simulations to control the convergence evolution and were slowly increased up to 0.9 .

The solution was considered converged when variation of the wall shear stress was inferior to $1 \%$, and the residue of all conservation equations was inferior $10^{-5}$. 
Table 3.3 - Conservation Equations Terms, $\boldsymbol{\kappa}-\boldsymbol{\varepsilon}$ Models

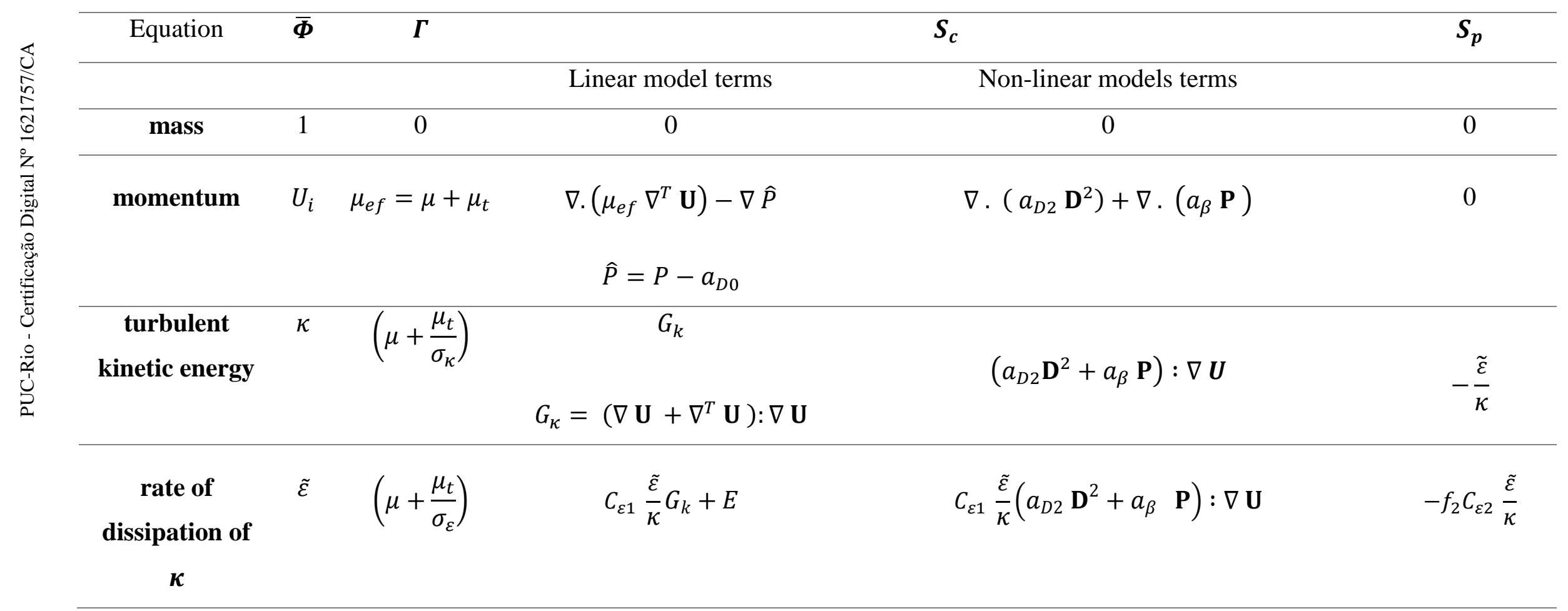




\section{Results}

To evaluate the different linear and non-linear RANS models, the classical plane channel flow problem was selected, as high-quality DNS databases are available in the literature. Here the DNS data of Thais et al. (2012), and of Lee and Moser (2015) were selected.

The problem of interest is a fully developed 2D turbulent flow in a horizontal plane channel, with a separation equal to $2 H$ between the plates, and length $L$. Due to the symmetry, it is sufficient to solve the problem in one half domain, as shown in Fig. 4.1. The main flow direction is referred to as $x$ while the normal direction is $y$. The lower boundary is the wall (with no-slip condition) while the upper boundary is the symmetry plane.

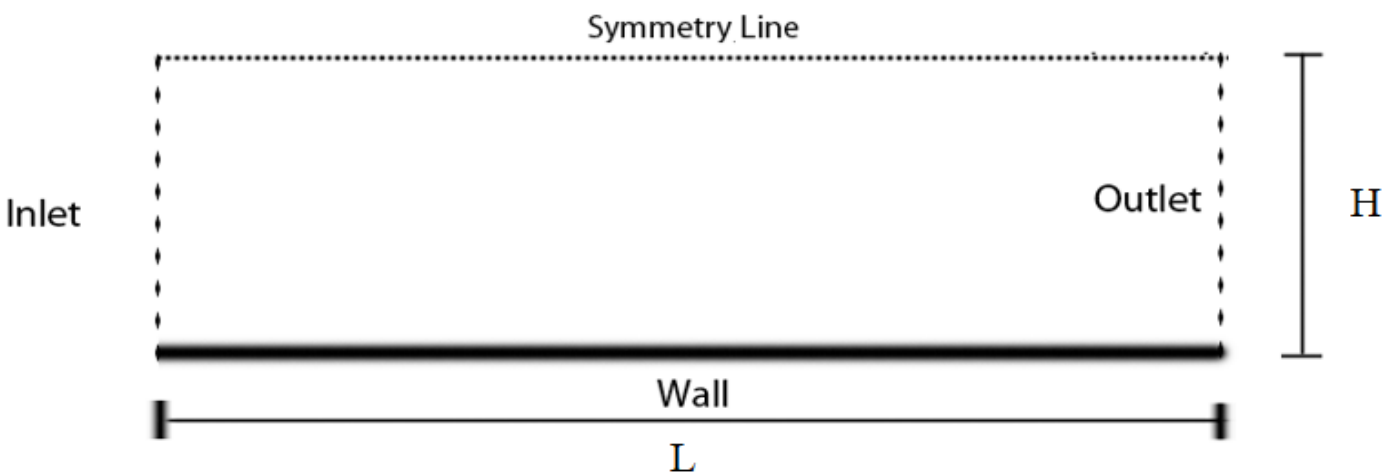

Figure 4.1 - Simulation's Geometry

Since the flow is fully developed, there is no variation of the velocity field in the axial direction, resulting in null acceleration. Therefore, there is a force balance equilibrium, i.e., the pressure force is equal to the viscous force

$$
\frac{\partial p}{\partial x} H=\tau_{w}
$$

The problem was solved as $2 \mathrm{D}$, with a periodic boundary condition in the $\mathrm{x}$ direction, i.e, all variables at the outlet are equal to the inlet quantities, and the pressure is defined as:

$$
p=-\beta x+\hat{p}
$$

where $\beta=\tau_{w} / H$ is constant coefficient to account for the pressure drive. This 
constant forcing is added at the $x$-momentum equation, and the periodic boundary is imposed to $\hat{p}$.

The flow is governed by the friction Reynolds number

$$
R e_{\tau}=\frac{\rho u_{\tau} H}{\mu}
$$

Table 4.1 shows the different Reynolds number, domain size, number of grid points, mesh resolution of the DNS data base of Thais et al. (2012), and of Lee and Moser (2015).

Table 4.1 - Parameters of the DNS data base of Thais et al. (2012), and Lee and Moser (2015)

\begin{tabular}{|l|c|c|c|c|c|c|}
\hline & $R e_{\tau}$ & $L_{x} \times L_{z} / H$ & $N_{x} \times N_{y} \times N_{z}$ & $\Delta \mathrm{x}^{+}$ & $\Delta \mathrm{y}_{\max }^{2}$ & $\Delta z^{+}$ \\
\hline \multirow{2}{*}{$\begin{array}{l}\text { Thais } \text { et } \\
\text { al. }\end{array}$} & 395 & $8 \pi \times 1.5 \pi$ & $1014 \times 257 \times 256$ & 9.6 & 7.9 & 7.2 \\
\cline { 2 - 7 }$(2012)$ & 590 & $8 \pi \times 1.5 \pi$ & $1536 \times 257 \times 512$ & 9.6 & 0.4 & 5.4 \\
\cline { 2 - 7 } & 1000 & $8 \pi \times 1.5 \pi$ & $1536 \times 513 \times 768$ & 12.3 & 8.4 & 6.1 \\
\hline $\begin{array}{l}\text { Lee and } \\
\text { Moser } \\
(2015)\end{array}$ & 1994 & $8 \pi \times 3 \pi$ & $4096 \times 768 \times 3072$ & 12.3 & 0.191 & 6.2 \\
\cline { 2 - 7 } & 5186 & $8 \pi \times 3 \pi$ & $10240 \times 1536 \times 7680$ & 12.7 & 0.498 & 6.4 \\
\hline
\end{tabular}

To discretize the problem, a non-uniform grid was defined in the normal direction. Since the flow is periodic in the x-direction, a small domain with length $L=H$ was defined with only 4 nodal points. Although, only one point could have been imposed, 4 points were defined just to facilitate handling the data. As shown in Appendix C, a grid with 257 points in the normal direction was sufficient to guarantee mesh independent results.

The main variables examined in this work are the mean axial velocity and all Reynolds stress components. The turbulent quantities $\kappa$ and $\varepsilon$ were also compared with the DNS data. Initially, a comparison of performance of available linear $\kappa-\varepsilon$ models is performed. The best model is selected and it is employed to be compared with the non-linear models.

Before presenting the comparison between the models, it is important to mention that the turbulent quantities are assessed through a direct comparison with the DNS data. However, for the mean axial velocity field, a reference DNS data was 
obtained by solving the momentum equations, imposing the DNS Reynolds stress.

\subsection{Reference Velocity Field}

Thompson et al., (2016) have presented a careful evaluation of statistical errors in DNS data of plane channel flow. It was shown that depending on the convergence criteria employed, different variables are better represented. Based on their work, the DNS reference velocity field was created, obtained by solving the 2D momentum equation imposing the turbulent shear stress from the DNS data instead of modeling it with Eqs. 3.16 or 3.30 .

With this approach, the reference velocity field (entitled 'DNS'- reference for short) corresponds to the best velocity profile that can be obtained in a $2 \mathrm{D}$ solution that target the DNS Reynolds tensor.

To impose the DNS Reynolds stress data to solve the momentum equation, the same number of grid points as the DNS simulation is desired. However, due to implementation details of the OpenFOAM nodes and the nodes position of the DNS data, two additional points (one near the wall and the other near the symmetry plane) were added. Details of the procedure employed here are given in Appendix C.

Figure 4.2 shows the comparison of the DNS velocity profile with the velocity reference field obtained for $\mathrm{Re}_{\tau}=1000$. From the plot it can be noticed that the reference field presents a very slight deviation from the DNS data.

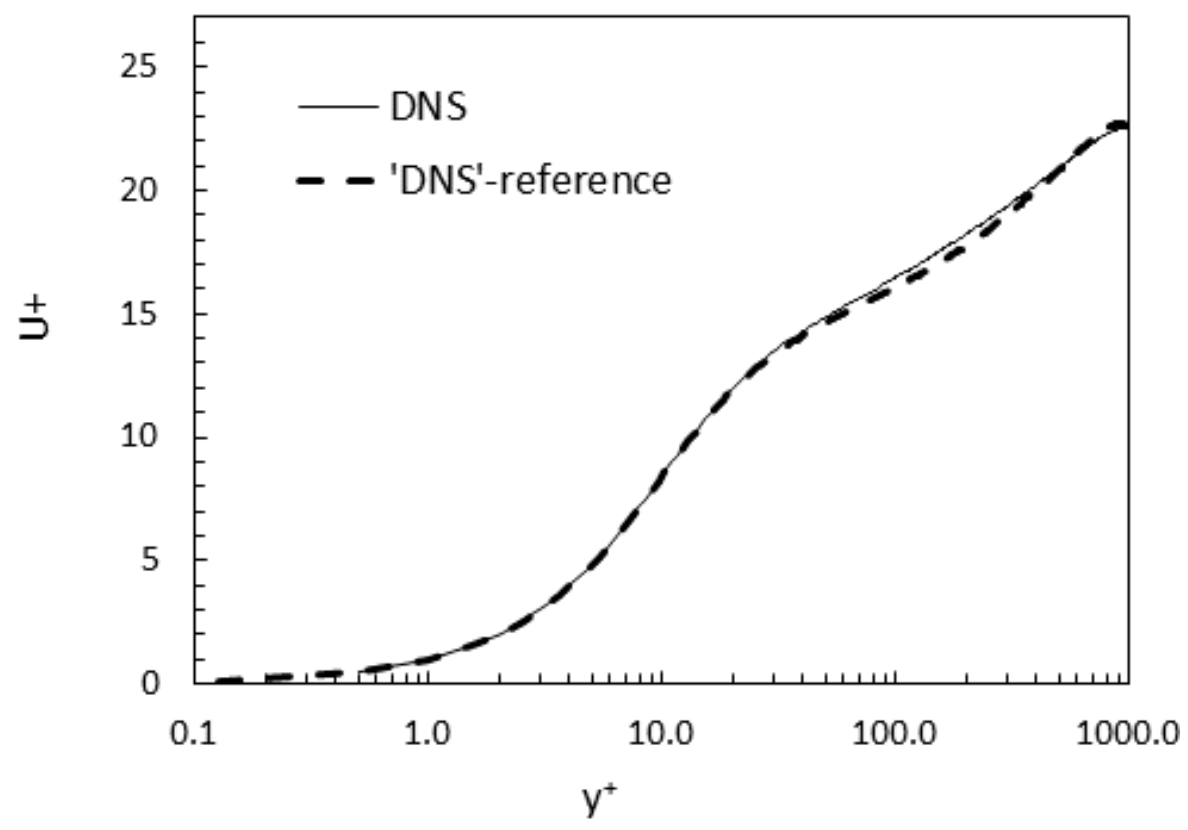


Figure 4.2 - Comparison of Velocity Profile from the DNS data with Reference field for $\boldsymbol{R e}_{\boldsymbol{\tau}}=1000$

\subsection{K- $\varepsilon$ Linear Models Results}

An evaluation of the selected linear models is presented here. The selected models were: Lam Bremhorst (Lam \& Bremhorst, 1981), Launder Sharma (Launder et al., 1977), Lien Leschziner (Lien \& Leschziner, 1993) and Modified Rodi Mansour (Michelassi et al., 1993).

At the present work, the modified Rodi Mansour model was implemented in OpenFoam, since Nieckele et al. (2016) have recommended that a damping viscosity coefficient $f_{\mu}$ (shown in Table 3.1) be applied to the linear term of the Reynolds stress, for all models they have evaluated employing an analysis a priori of the models. They also recommend that the coefficient $C_{\mu}$ should be set as 0.07, when they compared its distribution with Thais et al (2012) DNS data for different Reynolds number. In the present work, a new evaluation of this coefficient was performed, and it is shown in Appendix A.1. The value $C_{\mu}=$ 0.075 was considered the best value for the model and was applied in all analysis that follow.

Figure 4.3 shows a comparison of the results for mean axial velocity obtained with the four selected linear models with the reference 'DNS' profile, for $R e_{\tau}=$ 1000. It can be seen that Lauder Sharma, Lien Leschziner models have similar profiles that super estimates the DNS Reference velocity. The Lam Bremhorst was the one with worst velocity evaluation. Meanwhile the Rodi Mansour Modified shows great accuracy with the reference, although the maximum velocity at the channel center is under estimated.

The mean axial velocity obtained with Modified Rodi Mansour model was also compared with the log law (Eq. 3.25) and linear profile $\left(u^{+}=y^{+}\right)$in the viscous layer in Figure 4.4. It can be seen once again, the excellent agreement of Rodi Mansor data with the DNS Reference data. It can be observed that the profile can be evaluated by the linear behavior when $y^{+}<5$ and by the $\log$ law when $y^{+}>30$ just as discussed in Chapter 3 . The region between these two zones $\left(5<y^{+}<30\right)$ cannot be described by any of these formulations.

Figure 4.5 presents the shear Reynolds stress, $a_{x y}$, predictions of the four linear models for $R e_{\tau}=1000$, compared to the DNS data. Figure 4.6 compares the 
turbulent kinetic energy $\kappa$ and its dissipation $\varepsilon$, for the same models and Reynolds number.

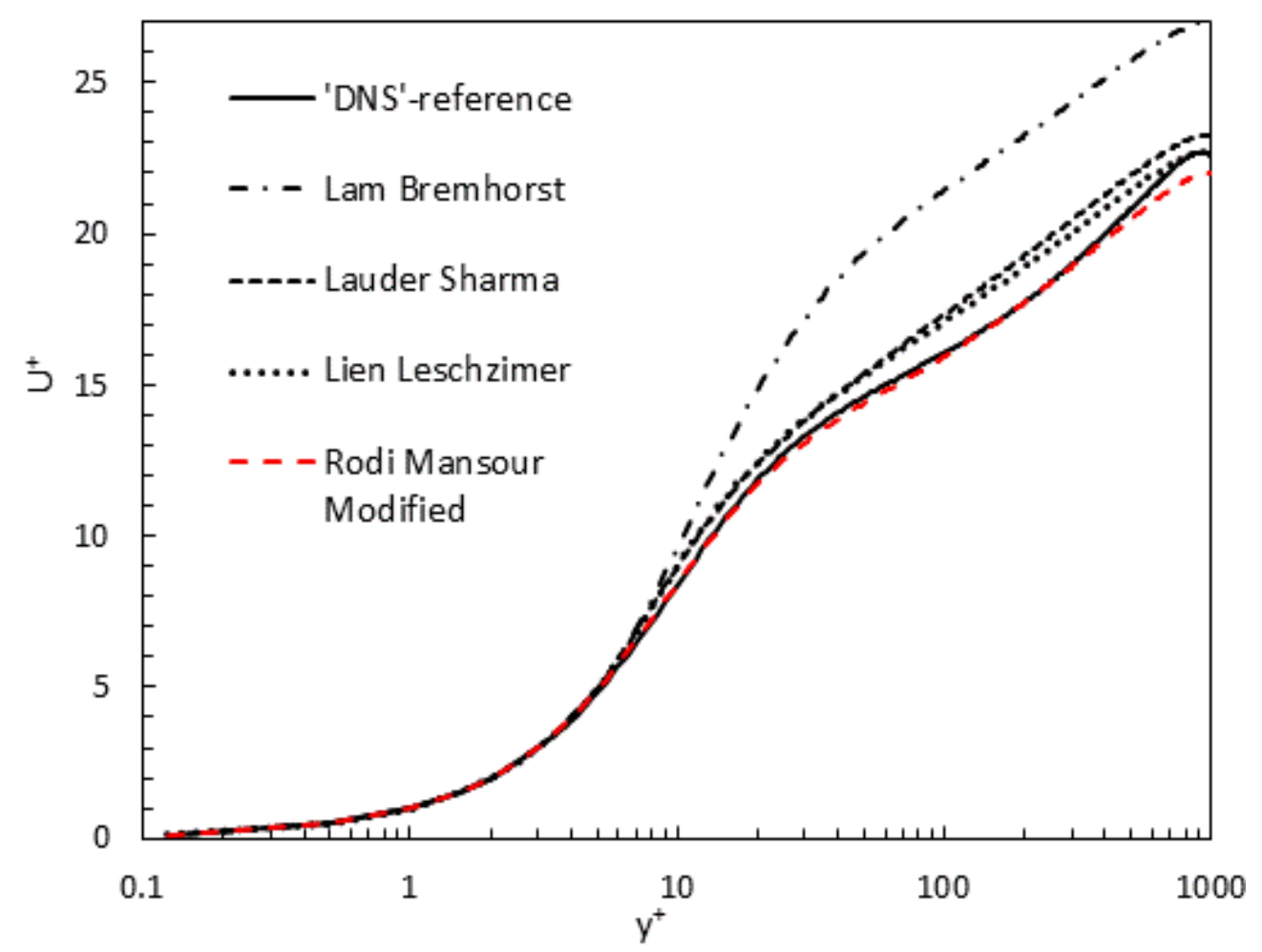

Figure 4.3 - Comparison of the mean axial velocity. Linear Models. $\boldsymbol{R} \boldsymbol{e}_{\boldsymbol{\tau}}=1000$.

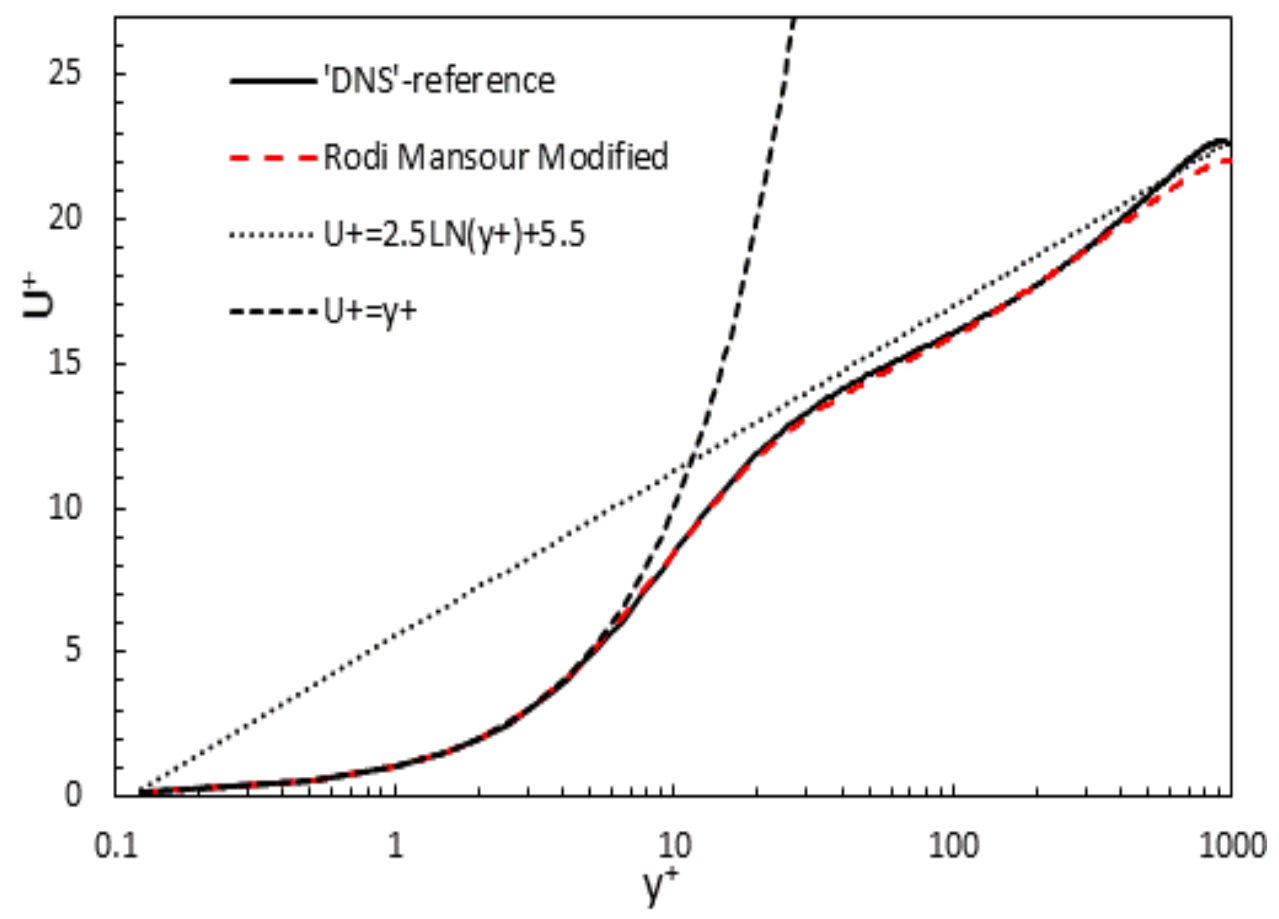

Figure 4.4 - Rodi Mansour Comparison with Linear an logarithmic behavior. 
From the $a_{x y}$ plot in Fig. 4.5 , it is possible to notice that all models present a region where the profile does not match the DNS data. For the Lam Bremhorst model, this region extends from $y^{+} \approx 2$ to $y^{+} \approx 70$, being the one with worst performance of all. The second worst model is the Launder Sharma with a region extending from $y^{+} \approx 2$ to $y^{+} \approx 20$. Lien Leschziner, provided the second-best profile where the problematic region is located when $y^{+}$values are between 2 and 10. The Rodi Mansour Modified, proved to be the best model with a mismatch region of $y^{+} \approx 2$ to 7 and even so, its values are much closer to the DNS profile than the other models. No linear model was able to evaluate the normal components of the Reynolds Stress Tensor.

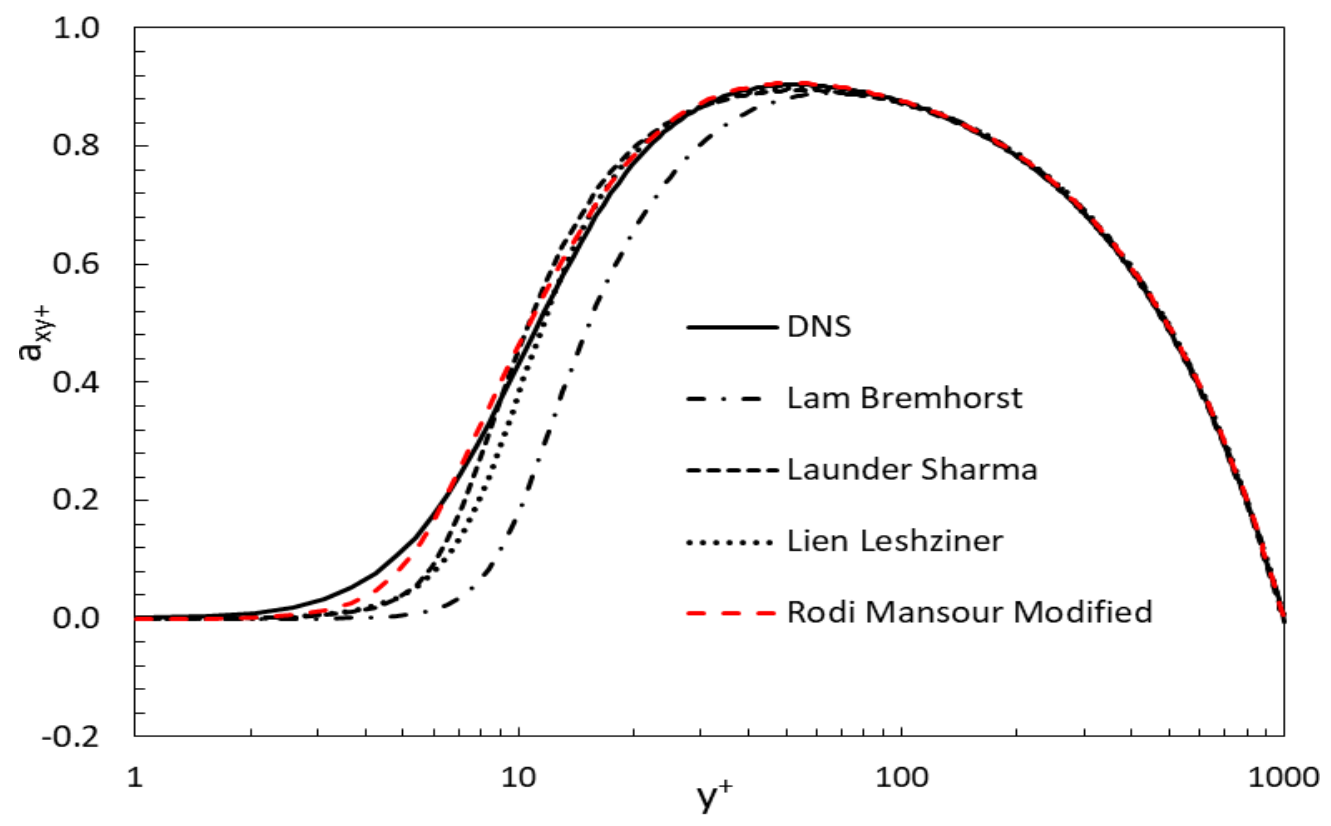

Figure 4.5 - Comparison of shear Reynolds stress. Linear Models. $\boldsymbol{R}_{\boldsymbol{\tau}}=1000$

For the $\kappa$ variable shown in Fig.4.6a, it can be seen that most models tend to underestimate its value except for the Modified Rodi Mansour, which presented the best prediction. In this case, the Launder Sharma showed the worst results followed by Lam Bremhorst and Lien Leschziner. It can also be seen in Fig. 4.6b that all models tend to disagree with each other with regards to the prediction $\varepsilon$. Each model predicts a different boundary $\varepsilon$ values at the wall surface. Once again, Modified Rodi Mansour model presents the best prediction, when compared with the DNS data.

Based on this analysis, the Modified Rodi Mansour linear model with $C_{\mu}=0.075$ was chosen to be used as the best representative of the class in the 
comparisons with the non-linear models that follows. Furthermore, the same $\kappa$ and $\varepsilon$ equations provided by this model (with its damping coefficients) were applied to obtain the needed $\kappa$ and $\varepsilon$ values for the other non-linear models.

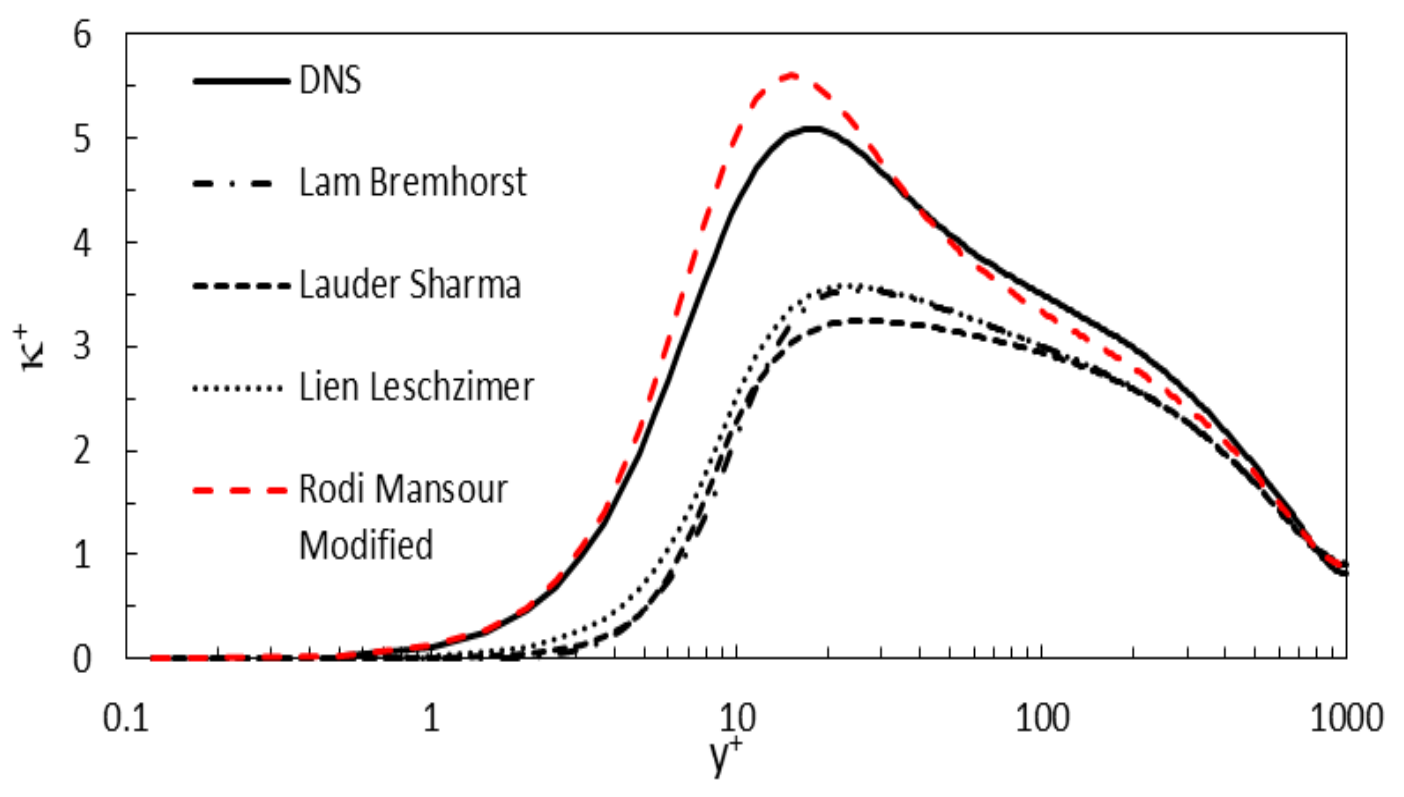

(a) Turbulent kinetic energy

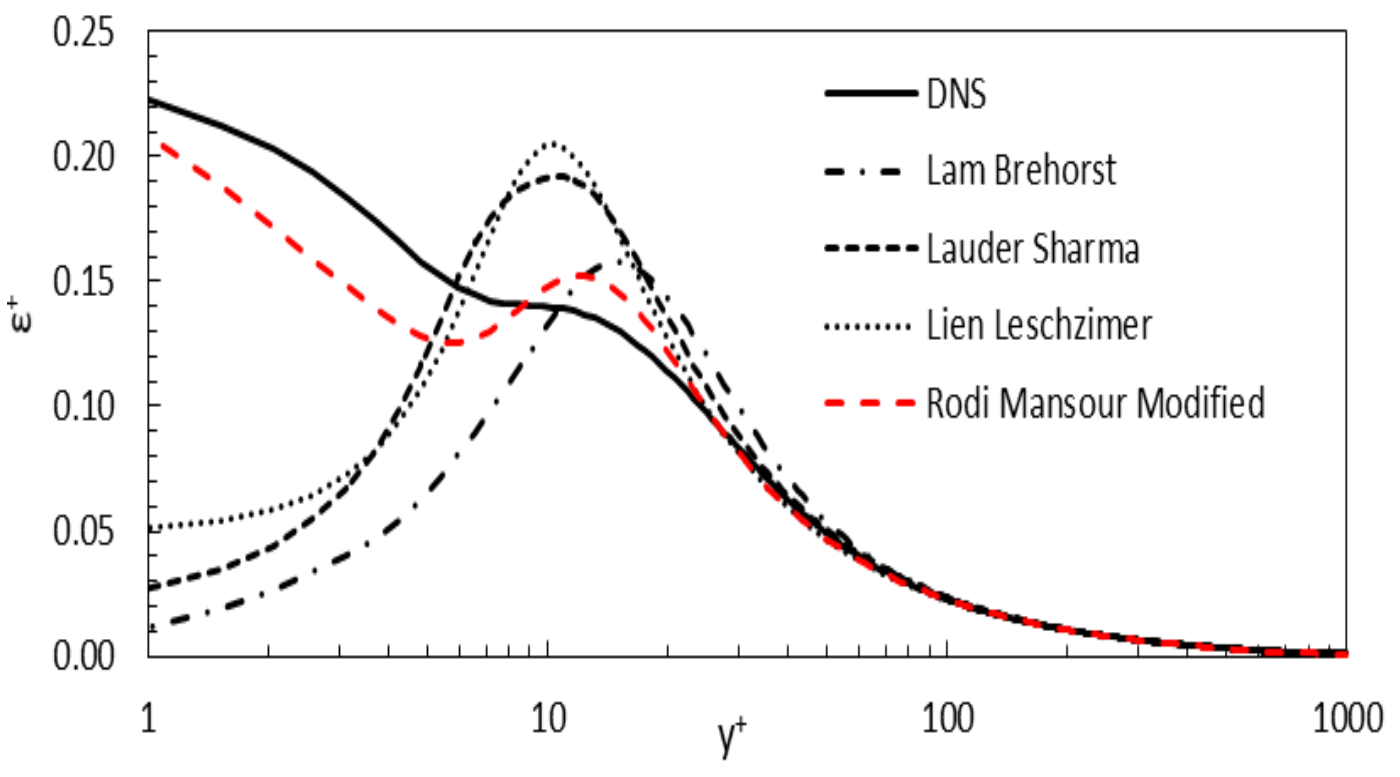

(b) Dissipation of turbulent kinetic energy

Figure 4.6 - Comparison of turbulent kinetic energy and its dissipation. Linear Models.

$$
\boldsymbol{R e}_{\boldsymbol{\tau}}=1000
$$

\subsection{K- $\varepsilon$ Non- Linear Models' Results}

This subsection presents the results obtained with the non-linear models II, III and IV, using the by $\kappa-\varepsilon$ coefficients nondimensionalization, for $R e_{\tau}=1000$. For this same Reynolds number, it is also shown a comparison between the best 
model of the previous test with the Lien Cubic non-linear model (Lien et al., 1991). Finally, the performance of the models for different number of Reynolds is shown.

\subsubsection{Models II, III and IV Results}

In this subsection a posteriori results of models II through IV are presented. As mentioned in the previous subsection these models are coupled with Rodi Mansour $\kappa$ and $\varepsilon$ equation. Figure 4.7 shows a comparison of the mean axial velocity of the different models with the reference DNS profile. Observing the velocity profile, all models present exactly the same velocity prediction, also agreeing with the linear Rodi Mansour model's (Model I) prediction. This behavior was expected, since all have exactly the same linear contribution. Moreover, they all closely match the DNS Reference velocity.

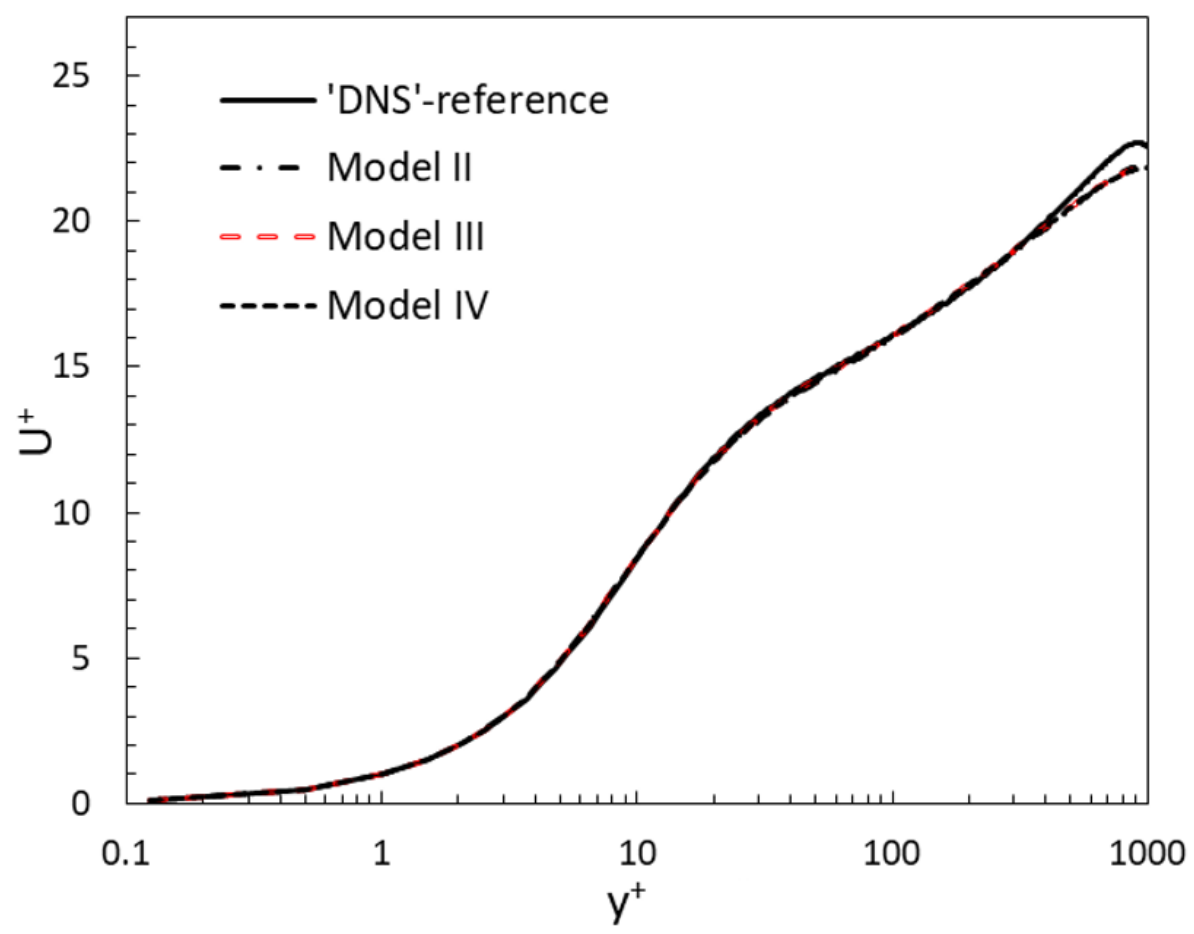

Figure 4.7 - Comparison of mean axial velocity. Non-Linear Models. $\boldsymbol{R} \boldsymbol{e}_{\boldsymbol{\tau}}=1000$.

The Reynolds tensor components are shown in Fig. 4.8. Note that all models predict exactly the same shear component $a_{x y}$. This behavior can be explained, by recalling that for the channel flow problem, $U=u(y) i$, all tensors shear 
components in $D_{x y}^{2}$ and $P_{x y}$ are null, therefore, only the linear term contributes for the shear Reynolds component $a_{x y}$.

The non-linear terms, however, present a significant influence in the prediction of the normal Reynolds components as shown in Fig. 4.8. Analyzing the performance of Model II, which includes the $D^{2}$ contribution, it can be seen that $a_{x x}$ is significantly underestimated. Further, examining the DNS data, $a_{y y}$ should be positive, and Model II predicts a profile equal to $a_{x x}$. This is expected because the $D_{i j}^{2}$ tensor is composed of only two equal normal components in the xx and yy directions for the channel flow. On the other hand, the prediction of $a_{z z}$ is reasonable, slightly over-estimated.

The non-linear contribution of Model IV is through the non-persistent tensor, which is related to the frame-invariant and objective version of the vorticity. This model's prediction is significant better than Model II for $a_{x x}$ and $a_{y y}$, both of them present the correct behavior. The former is slightly underestimated while the latter is over-estimated. The $a_{z z}$ component, however, could not be predicted by this model.

Model III, which presents contribution from $D^{2}$ and $P$, shows the best agreement with the DNS data. The combination of the two tensors, one increasing the normal stress contribution and the other reducing it, produces the best prediction of the normal components of the Reynolds tensor.

The prediction of the turbulent kinetic energy $\kappa$ and its dissipation $\varepsilon$ is shown in Fig.4.9. Very good agreement is obtained with all models, however, Model II overestimates the $\kappa$ peak value. The dissipation rate, $\varepsilon$, prediction is also very accurate and significantly superior than the values obtained with several of the linear models. Comparing with Model I (modified Rodi-Mansour) the results are equal in terms of accuracy. It should be mentioned here that both kinetic energy and its dissipation rate play an import hole in the prediction of the flow, and consequently the Reynolds tensor components. The influence of these fields to the solution can be observed in Appendix D.

One important conclusion is that all models' results follow the same trends observed in the a priori analysis of Nieckele et al. (2016), shown in Fig. 4.10. This analysis uses only noble data from the DNS while, in a posteriori simulations, numerical errors as well as errors associated with $\kappa$ and $\varepsilon$ modeling are presented. 
The simulations results, therefore, show that these errors are small enough so a posteriori analysis could have the same a priori behavior.

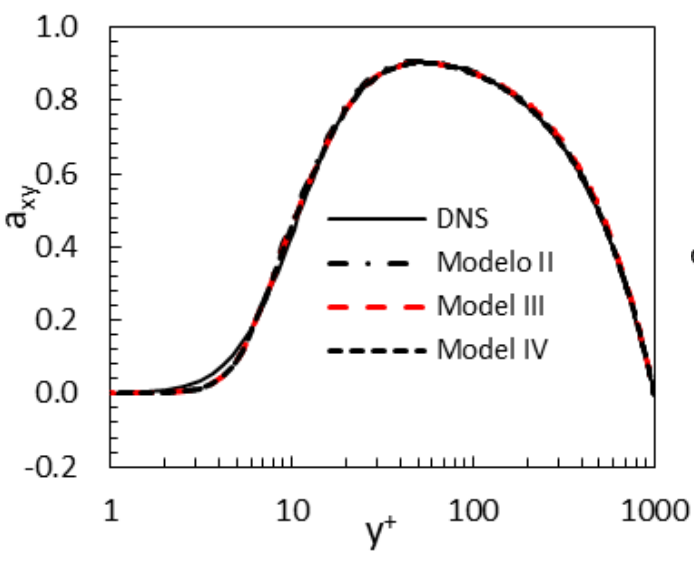

(a) $a_{x y}$

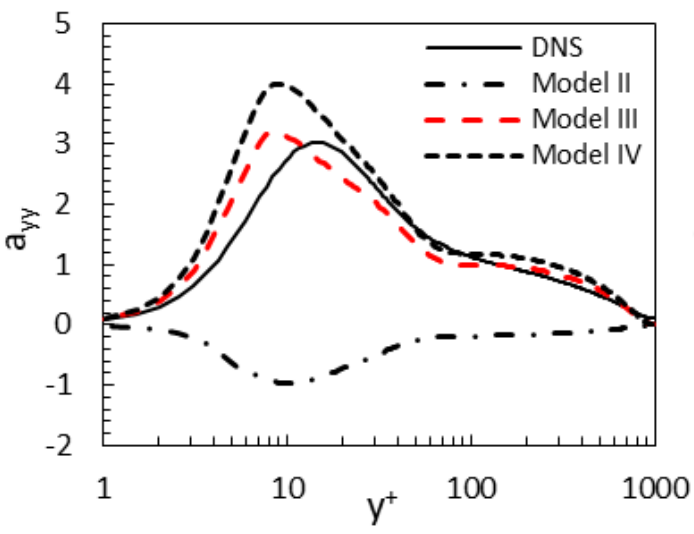

(c) $a_{y y}$

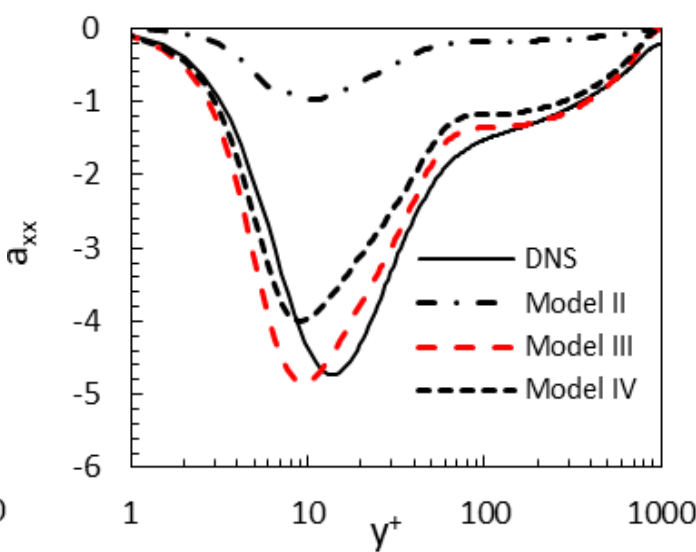

(b) $a_{x x}$

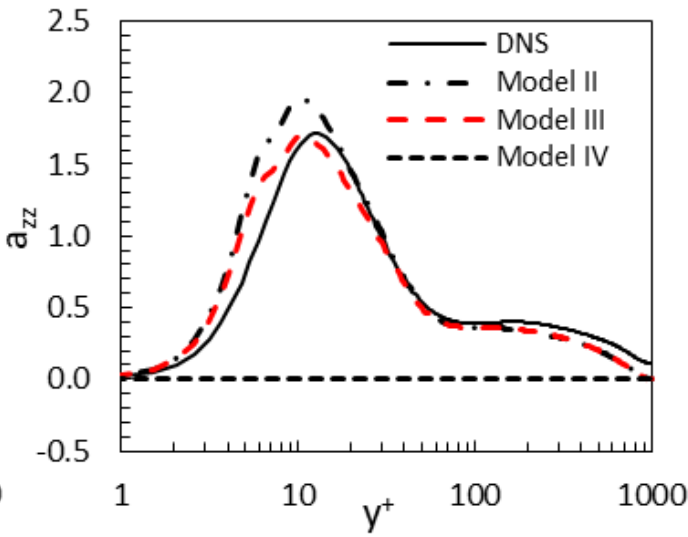

(d) $a_{z z}$

Figure 4.8 - Reynolds tensor components. Non-Linear Models. $\boldsymbol{R e}_{\boldsymbol{\tau}}=1000$

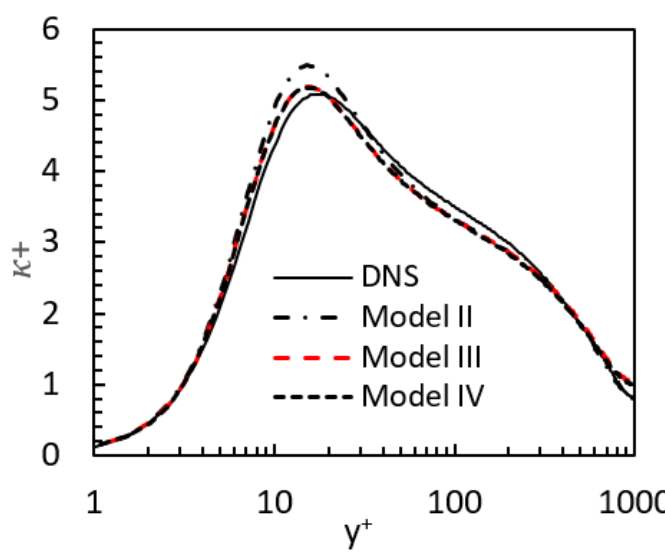

(a) $\kappa$, turbulent kinetic energy

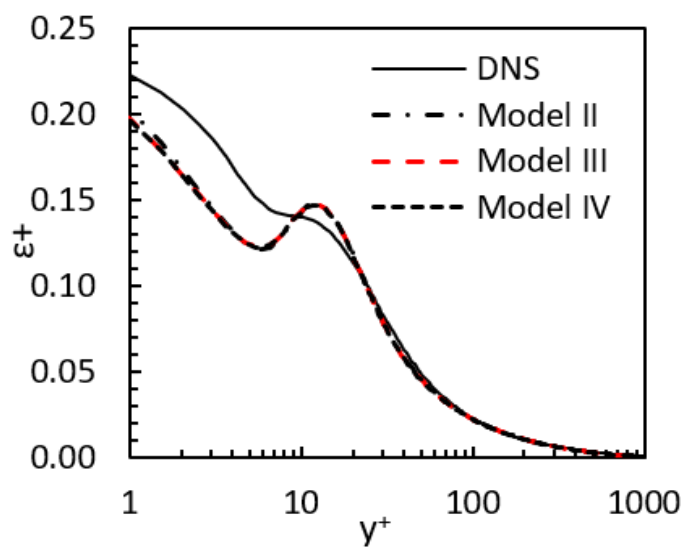

(b) $\varepsilon$, dissipation of $\kappa$

Figure $4.9-$ Turbulent kinetic energy and its dissipation. Non-Linear Models. $R e_{\tau}=1000$ 

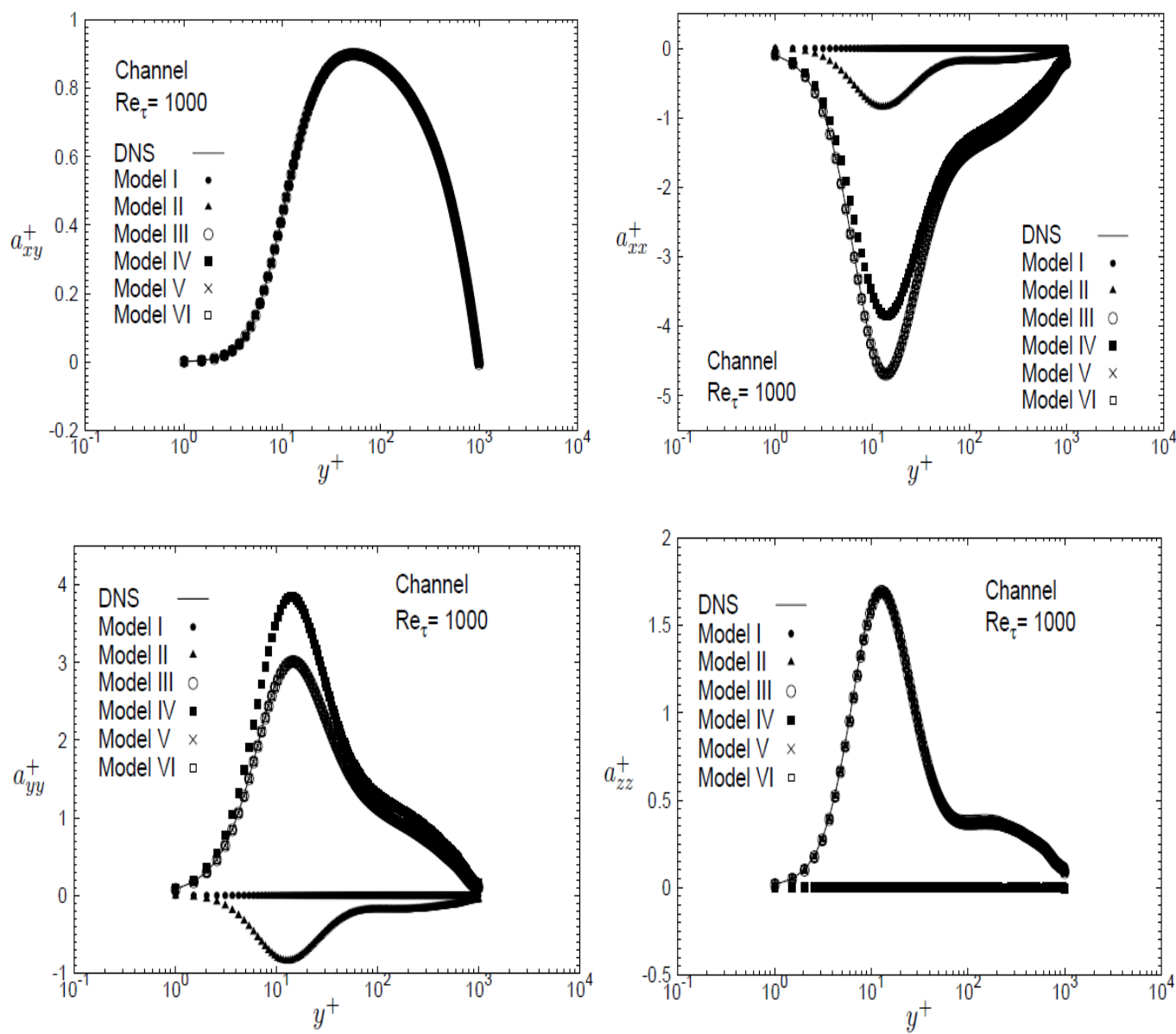

Figure 4.10 - A priori analysis of the six models proposed by (Nieckele et al., 2016) in a Channel Flow with $R e_{\tau}=1000$

\subsubsection{Comparison with Literature Non-Linear Model}

From the previous analysis, Model III has shown the best prediction of the Reynolds Stress Tensor. Thus, it was selected to be compared with a non-Linear model from the literature. The chosen model was the Lien Cubic model (Lien et al. 1991) which is already implemented in OpenFoam 4.1 version. Figure 4.11 through Figure 4.13 show a comparison between these two models for the mean axial velocity profile, the Reynolds tensor components and the turbulent quantities $\kappa$ and $\varepsilon$. fields.

It is possible to observe that, for all computed fields, Model III has a better prediction capability. It can be seen in Fig. 4.11 that the mean axial velocity of Model III matches the DNS data, while Lien Cubic model overestimate it. Note also, in Fig. 4.12 and 4.13 that the Lien Cubic model not only underestimated $a_{x x}$, $a_{y y}$ and $a_{z z}$ and $\kappa$ but also wrongly generates the $\varepsilon$ profile decreasing near the wall. The $a_{x y}$ component does not adhere to the DNS data in a large region $\left(2<y^{+}<\right.$ 15)). As a result, the velocity profile is super estimated. 


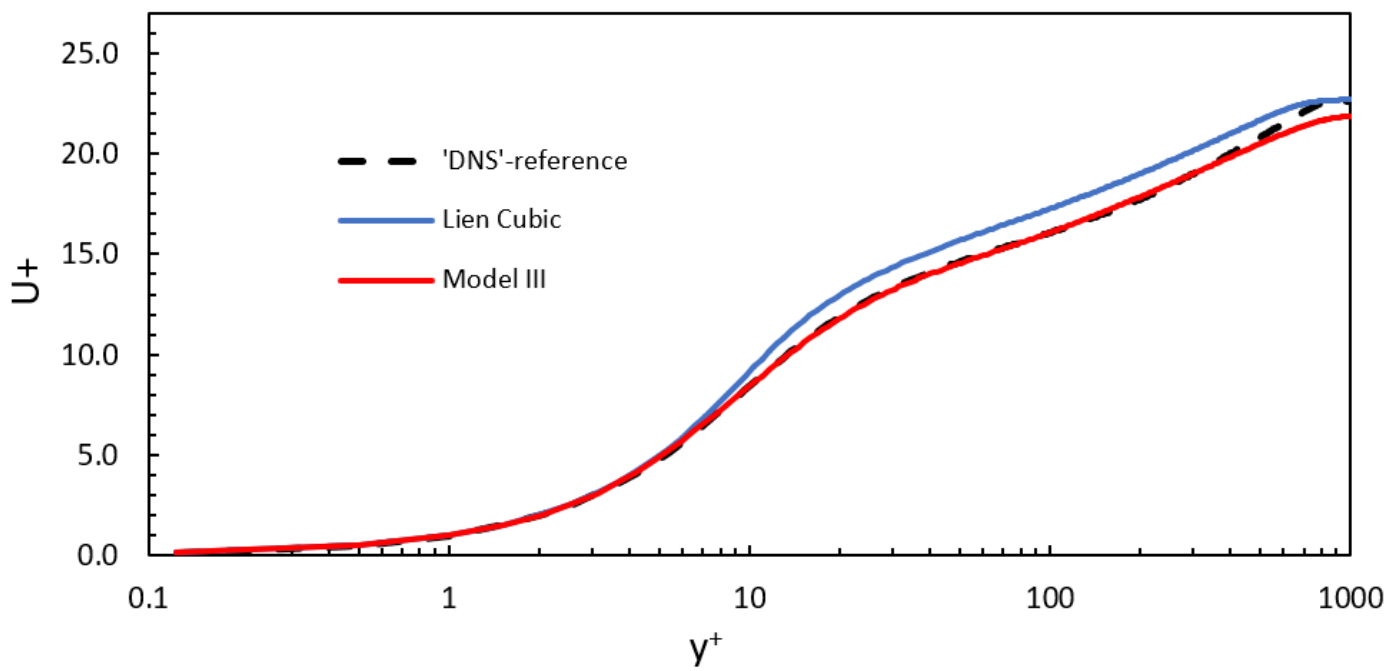

Figure 4.11 - Comparison of mean axial velocity. Model III versus Lien Cubic model. $\boldsymbol{R e}_{\boldsymbol{\tau}}=1000$.

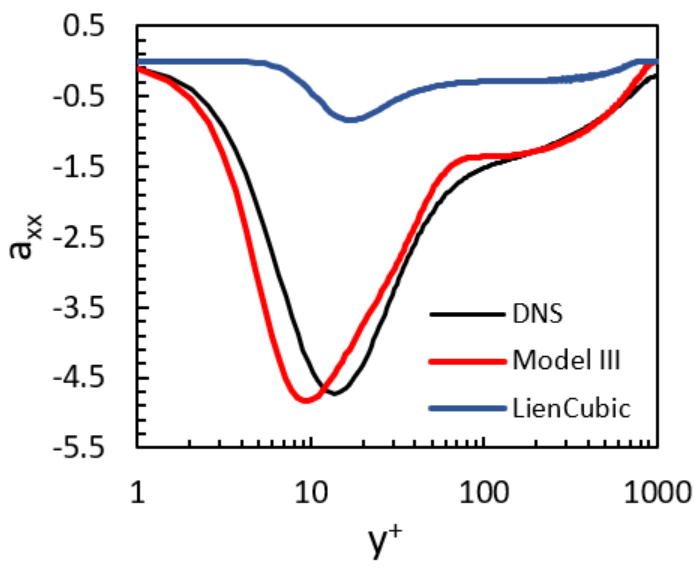

(a) $a_{x x}$

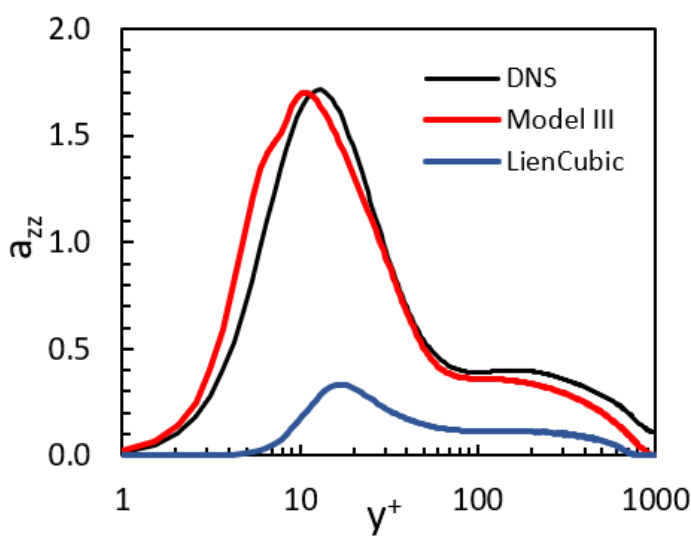

(a) $a_{z Z}$

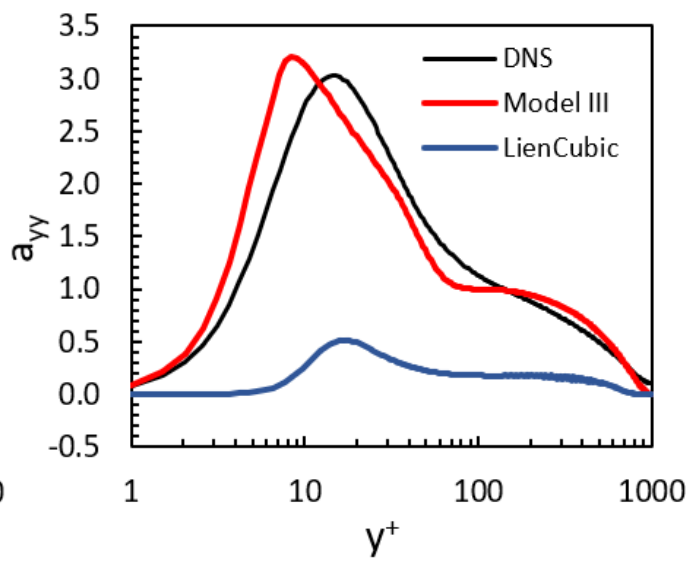

(b) $a_{y y}$

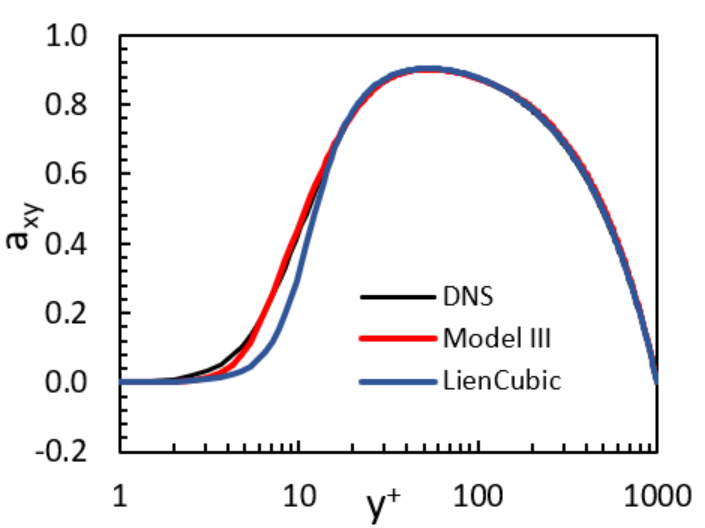

(b) $y$

Figure 4.12 - Reynolds tensor components. Model III versus Lien Cubic model.

$$
\boldsymbol{R e}_{\boldsymbol{\tau}}=1000
$$




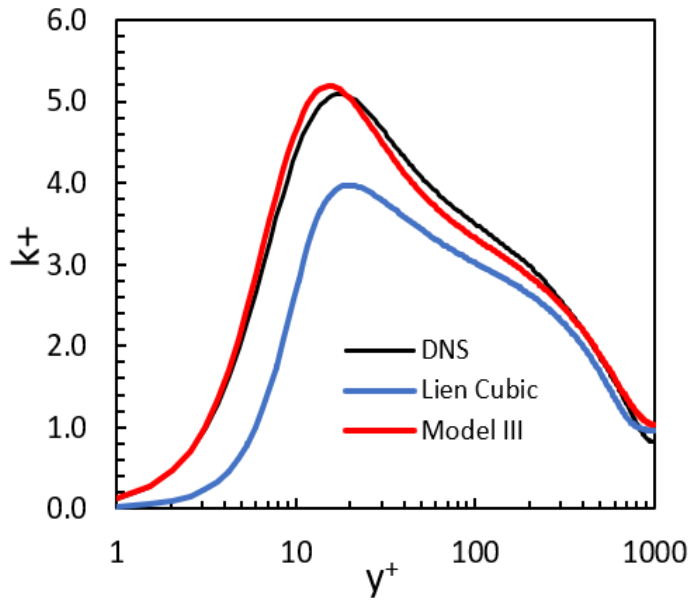

(a) $\kappa$, turbulent kinetic energy

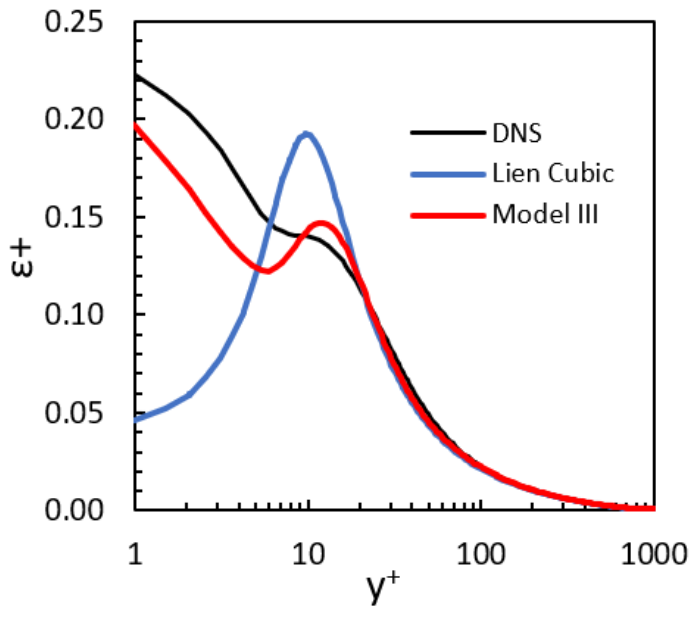

(b) $\varepsilon$, dissipation of $\kappa$

Figure 4.13 - Turbulent kinetic energy and its dissipation. MODEL III VERSUS Lien Cubic model. $R e_{\tau}=1000$

\subsubsection{Models' Results for Different Reynolds Numbers}

To verify the influence of the friction Reynolds number in the model analysis predictions, four more Reynolds number were investigated. Two inferior Reynolds number from Thais et al. (2012) DNS data base, $R e_{\tau}=395$ and $R e_{\tau}=590$ were selected. The other two cases were selected from Lee and Moser (2015) DNS data base, corresponding to higher Reynolds numbers $\left(R e_{\tau}=2000\right.$ and $\left.R e_{\tau}=5200\right)$.

The mean axial velocity is shown in Fig 4.14 while the shear Reynolds component of the Reynolds tensor $a_{x y}$ is shown in Fig. 4.15 and for all four Reynolds number. All models present very similar profiles for both variables, for each Reynolds number. The profiles are almost coincident, with a slight under estimation of the mean velocity in relation to the DNS data, but excellent agreement of the shear Reynolds stress, indicating universal behavior of the models, i.e., the models behavior is independent of the Reynolds number. 


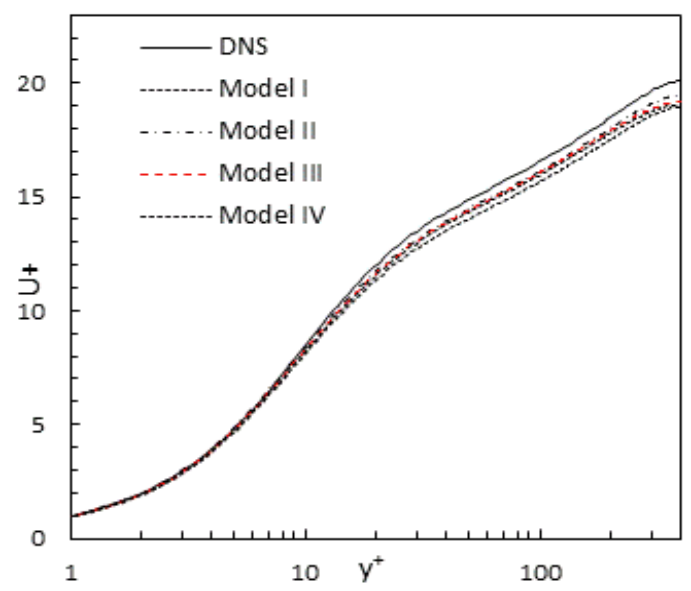

(a) $R e_{\tau}=395$

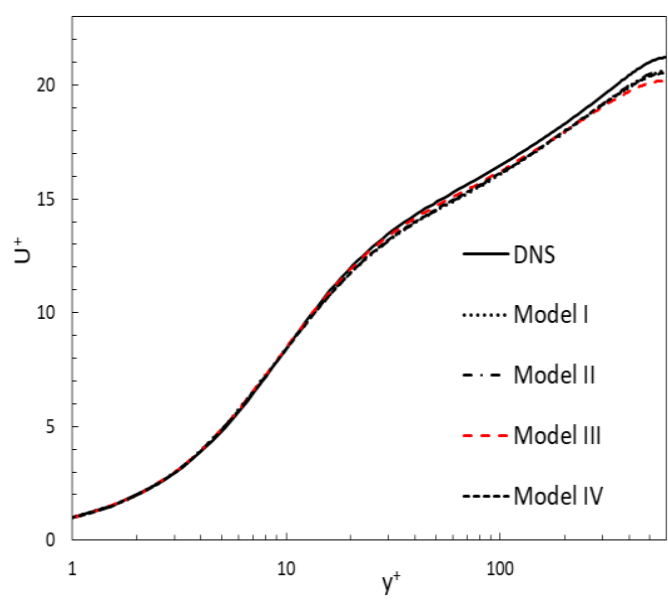

(b) $R e_{\tau}=590$

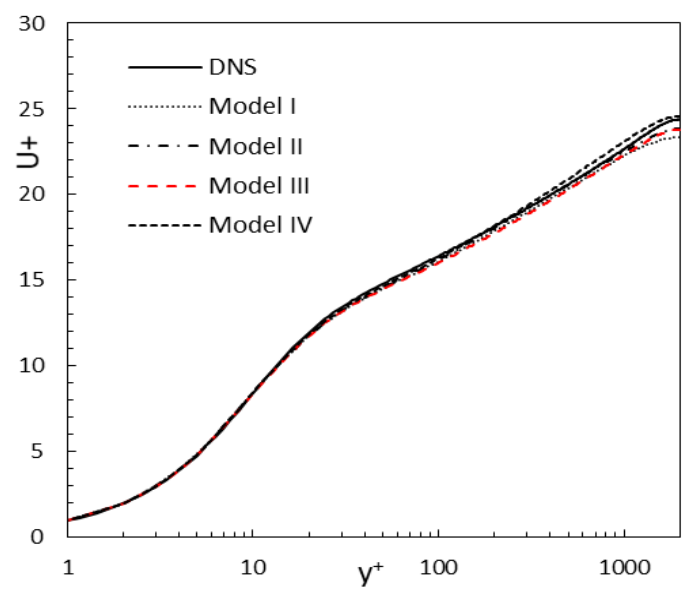

(c) $R e_{\tau}=2000$

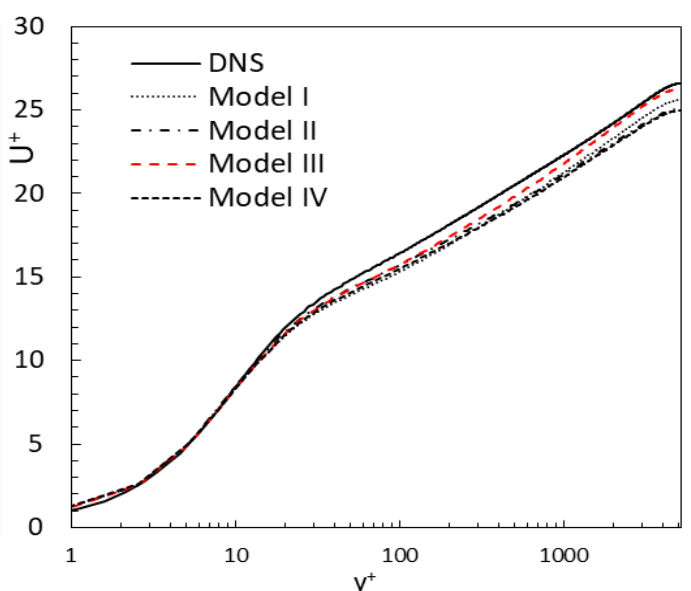

(d) $R e_{\tau}=5200$

Figure 4.14 - Comparison of mean axial velocity with DNS data. Different Reynolds numbers.

The behavior of the four models with respect to the prediction of the normal Reynolds stress tensor can be seen in Figs. 4.16 and 4.17. It can be seen the same behavior previously described. Model I (linear model) is unable to predict any normal component. Model II underestimates $a_{x x}$ and predicts equal $a_{y y}$ to $a_{x x}$, with the wrong sign, and overestimates $a_{z z}$. Model IV shows a better prediction of $a_{x x}$ and $a_{y y}$, but fails to predict $a_{z z}$. For all Reynolds numbers Model III has shown a good adherence to the DNS data, however, a slight deterioration of the results as Reynolds number increases is observed. 


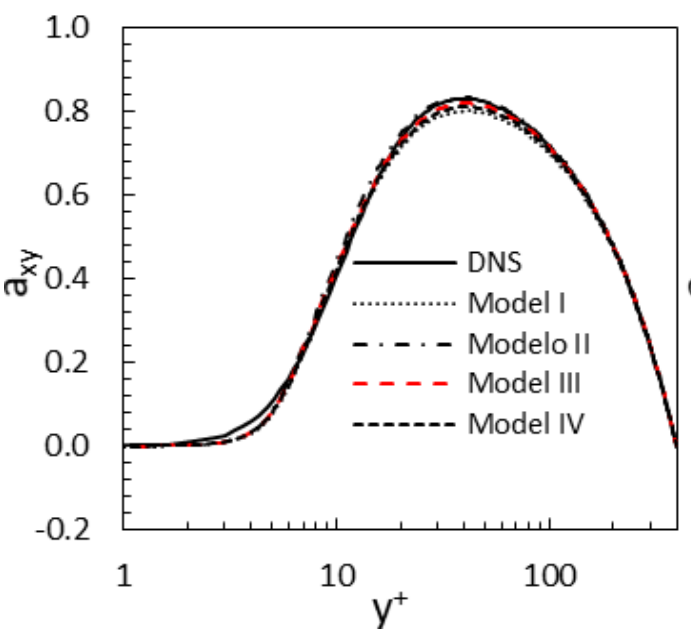

(a) $R e_{\tau}=395$

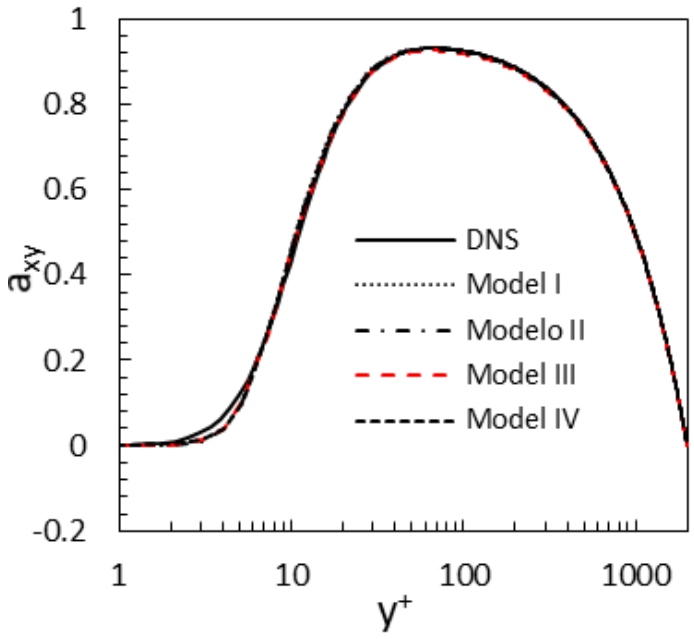

(c) $R e_{\tau}=2000$

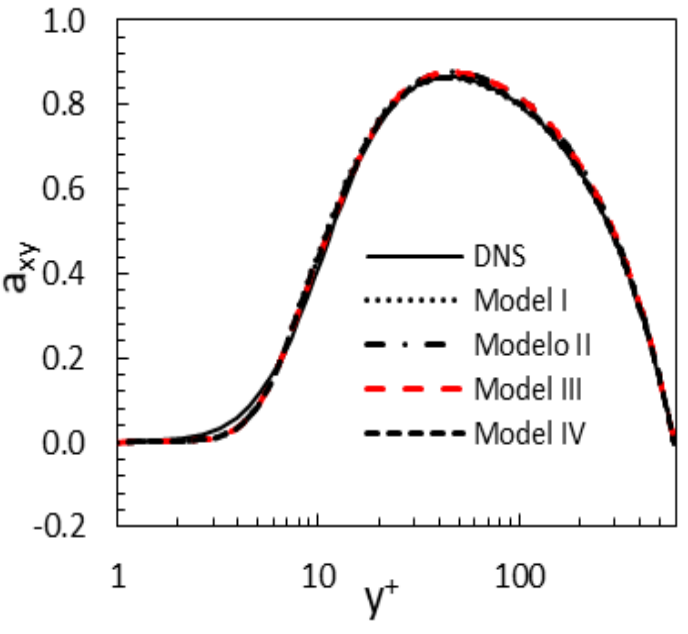

(b) $R e_{\tau}=590$

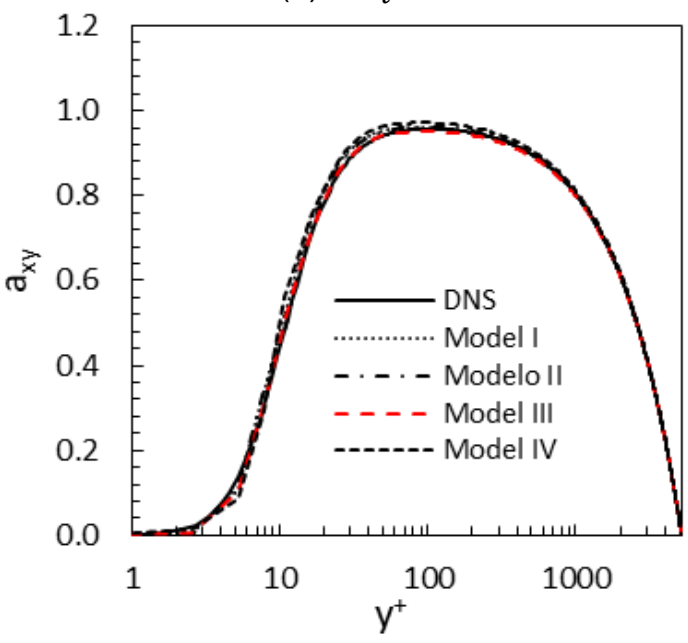

(d) $R e_{\tau}=5200$

Figure 4.15 - Comparison of the shear Reynolds tensor components $\boldsymbol{a}_{\boldsymbol{x} y}$ with DNS data. Different Reynolds numbers.

The performance of the models with respect to the turbulent kinetic energy and its dissipation rate is shown in Fg. 4.18. The models' predictions are equivalent, but a deterioration of the agreement is observed for the two higher Reynolds number, which can be explained by the larger difference also observed for the $a_{z z}$ component for the higher Reynolds numbers.

As a final remark, it is important to mention, that all models presented exactly the same behavior for all variables as obtained with the analysis a priori developed by Nieckele et al (2016). 


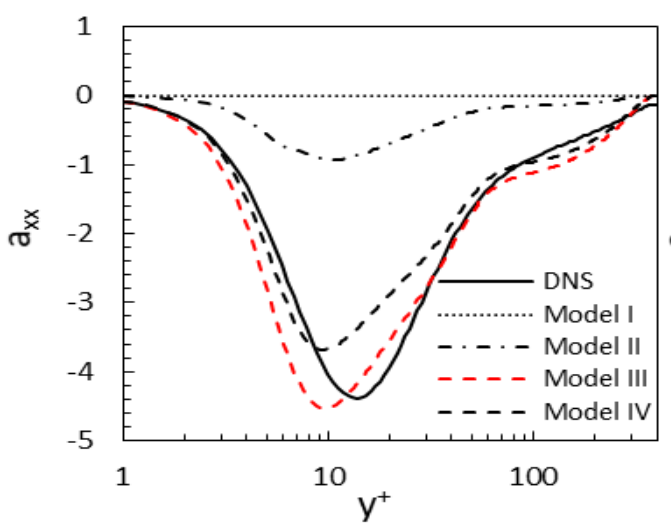

(a) $a_{x x} ; R e_{\tau}=395$

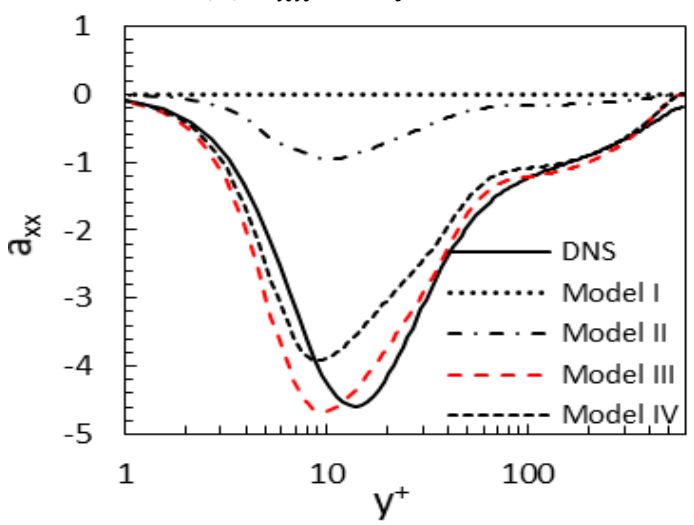

(b) $a_{x x} ; R e_{\tau}=590$

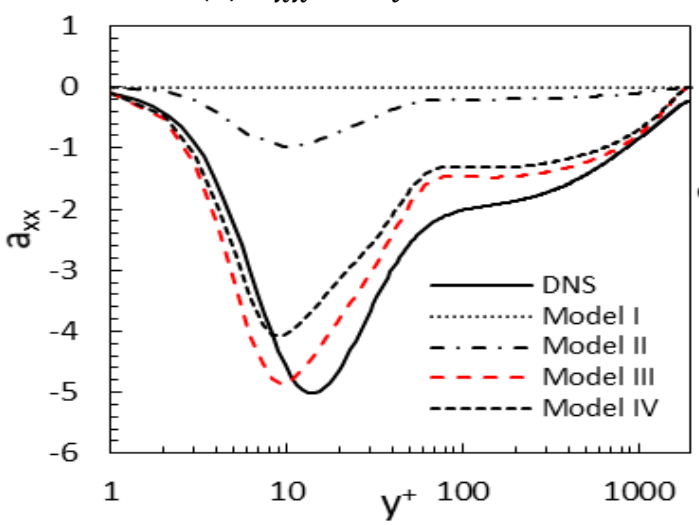

(c) $a_{x x} ; R e_{\tau}=2000$

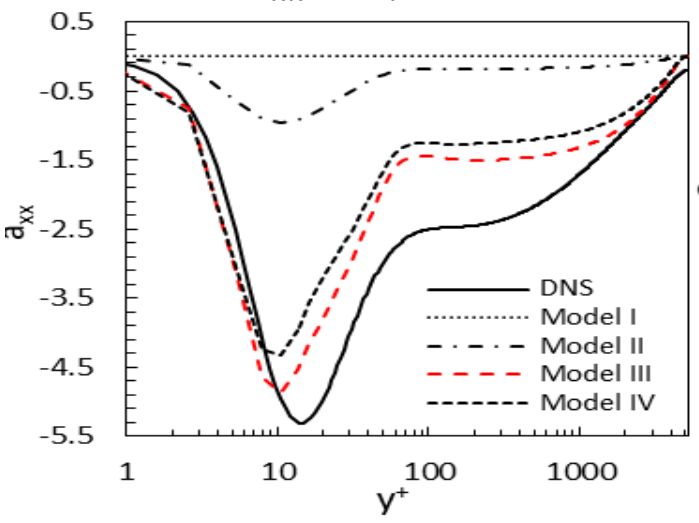

(d) $a_{x x} ; R e_{\tau}=5200$

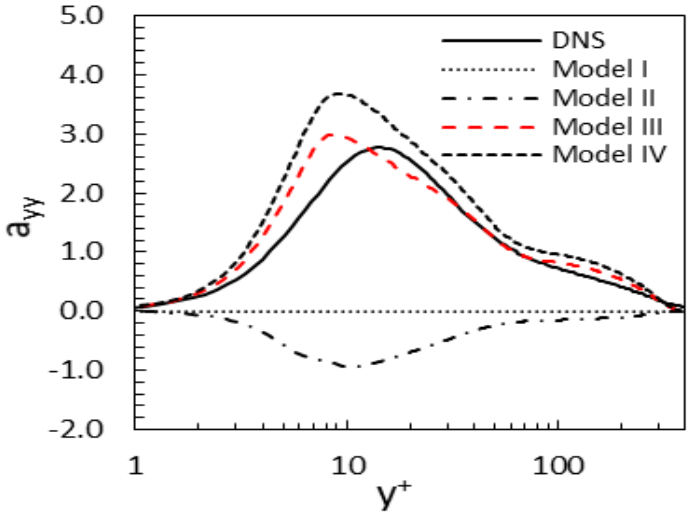

(e) $a_{y y} ; R e_{\tau}=395$

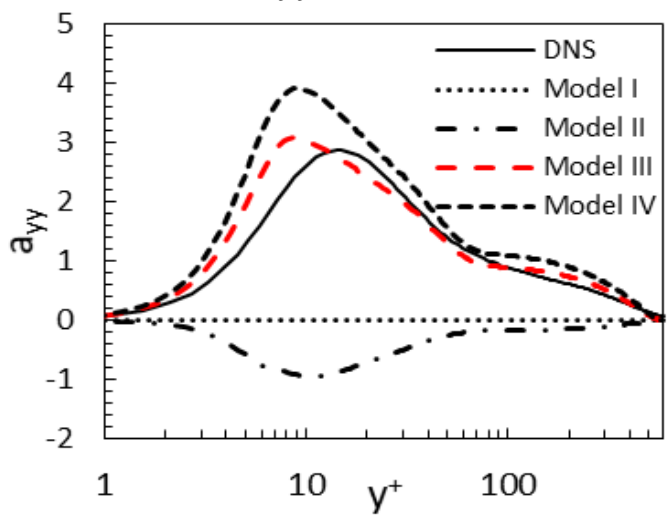

(f) $a_{y y} ; R e_{\tau}=590$

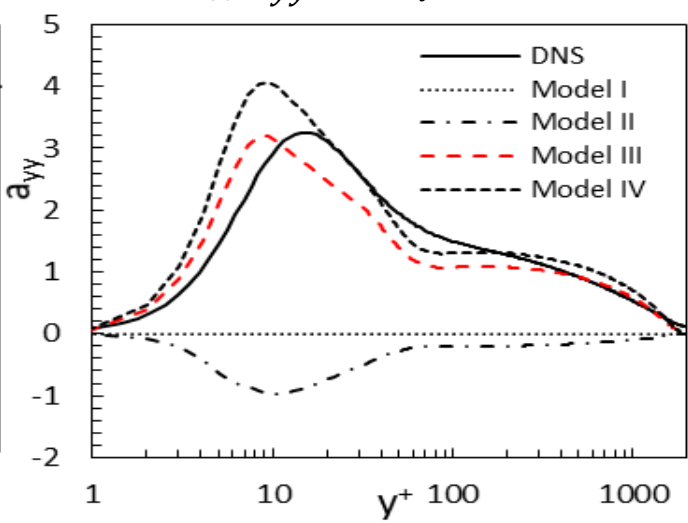

(g) $a_{y y} ; R e_{\tau}=2000$

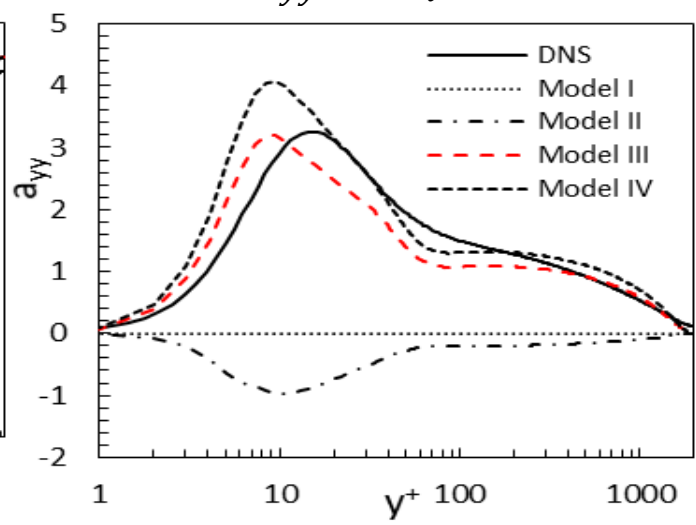

(h) $a_{y y} ; R e_{\tau}=5200$

Figure 4.16 - Comparison of the normal Reynolds tensor components $\boldsymbol{a}_{\boldsymbol{x} x}$ and $\boldsymbol{a}_{\boldsymbol{y} y}$ with DNS data. Different Reynolds numbers. 


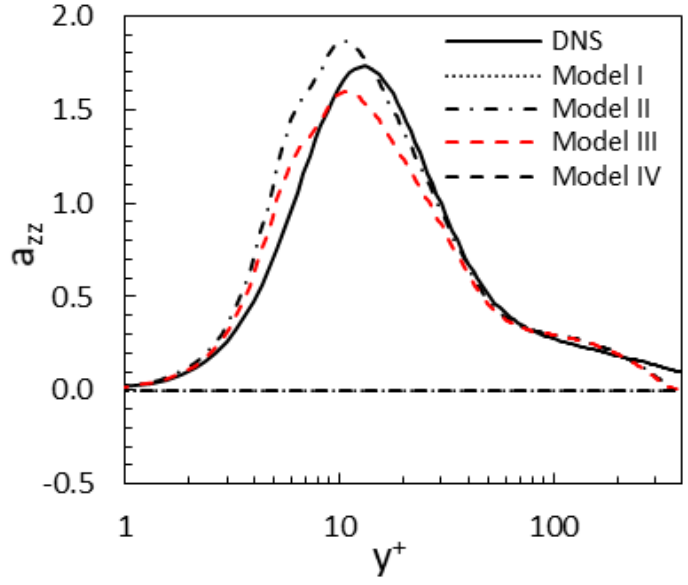

(a) $R e_{\tau}=395$

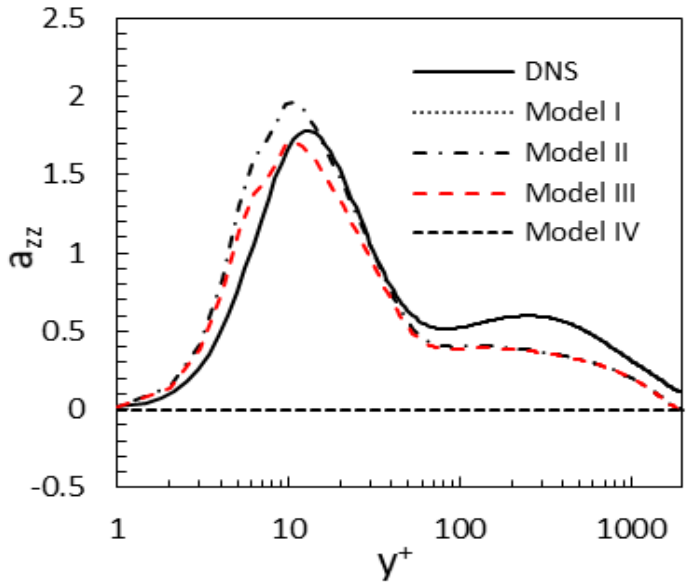

(c) $R e_{\tau}=2000$

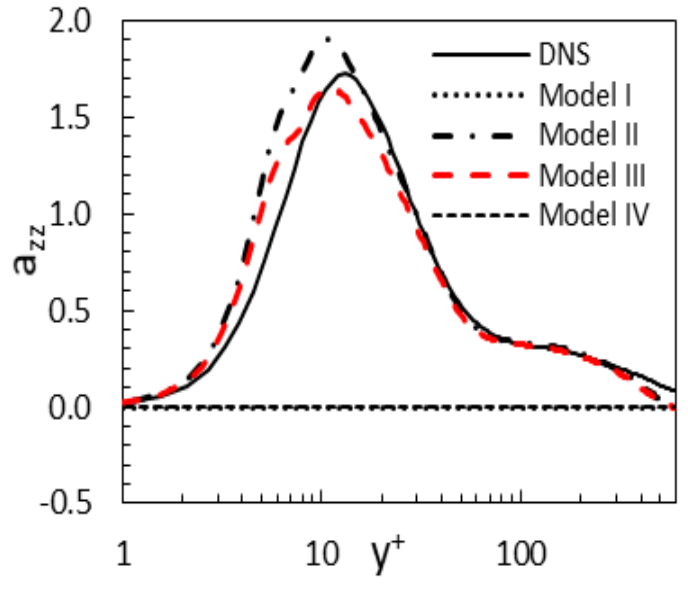

(b) $R e_{\tau}=590$

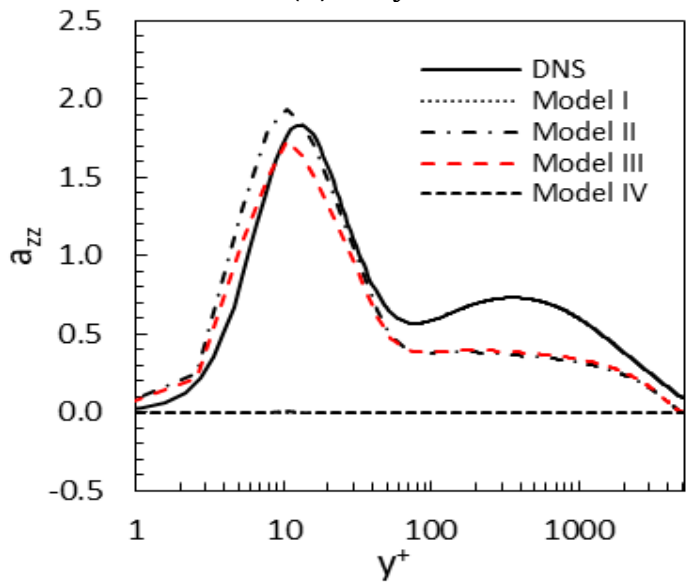

(d) $R e_{\tau}=5200$

Figure 4.17 - Comparison of the normal Reynolds tensor components $\mathbf{a}_{\mathrm{zz}}$ with DNS data. Different Reynolds numbers. 


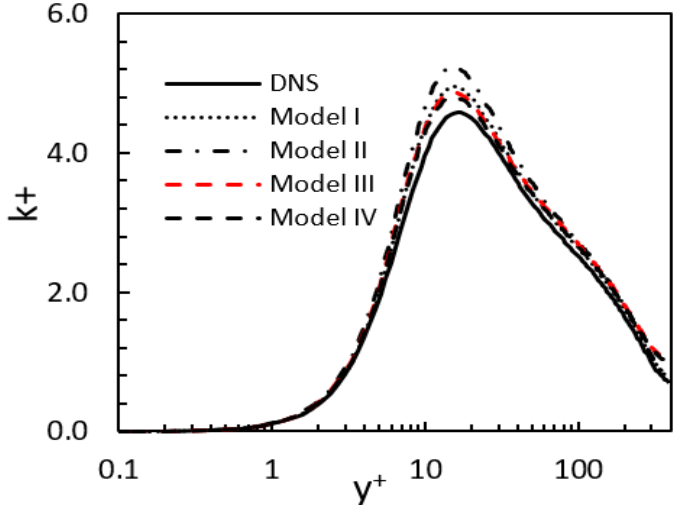

(a) $\kappa^{+} ; R e_{\tau}=395$

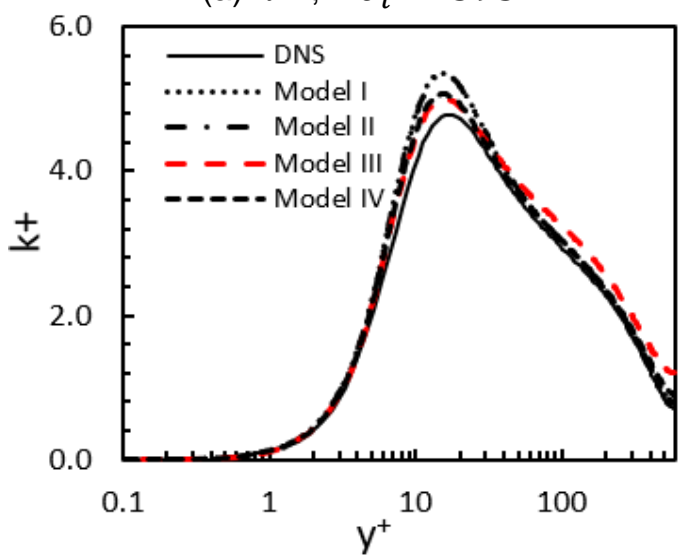

(b) $\kappa^{+} ; R e_{\tau}=590$

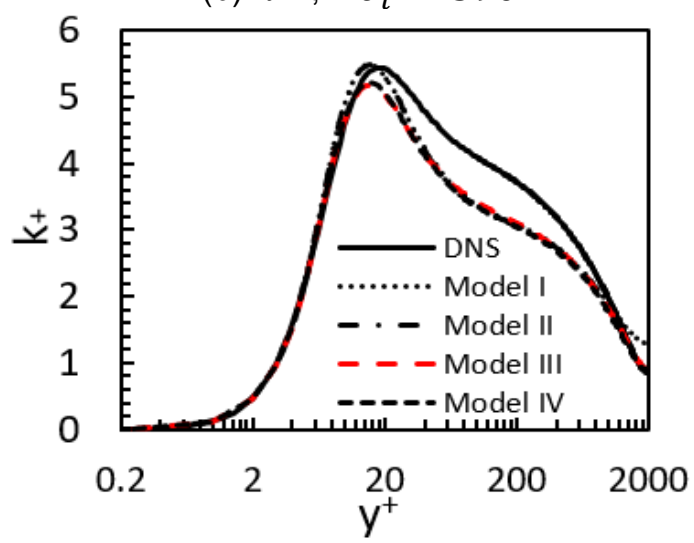

(c) $\kappa^{+} ; R e_{\tau}=2000$

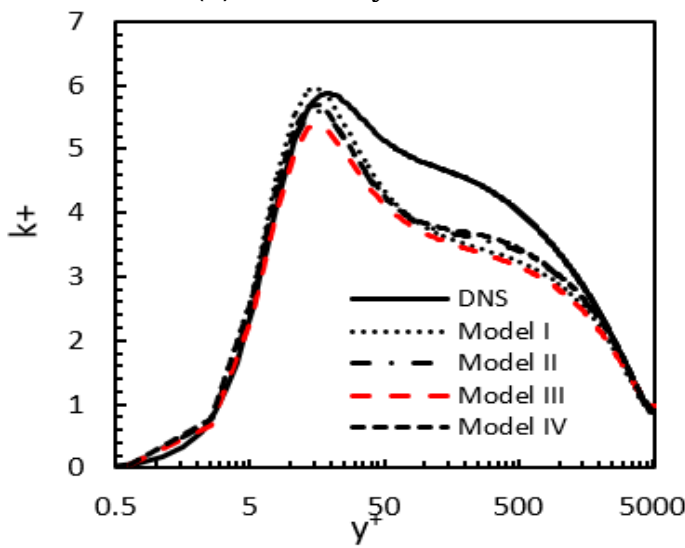

(a) $\kappa^{+} ; R e_{\tau}=5200$

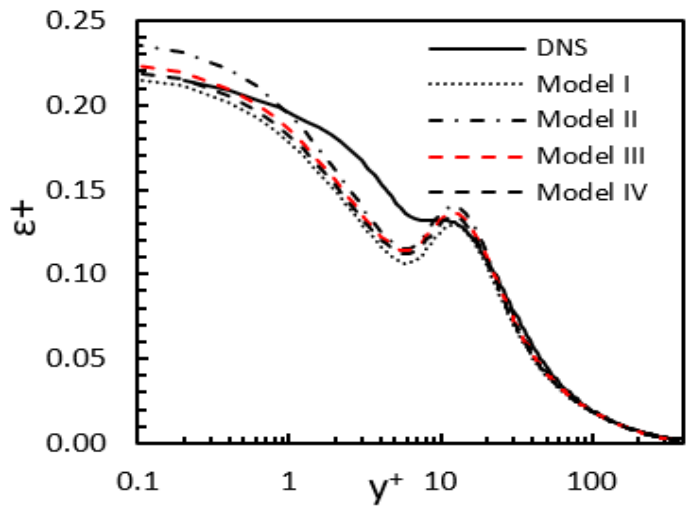

(e) $\varepsilon^{+} ; R e_{\tau}=395$

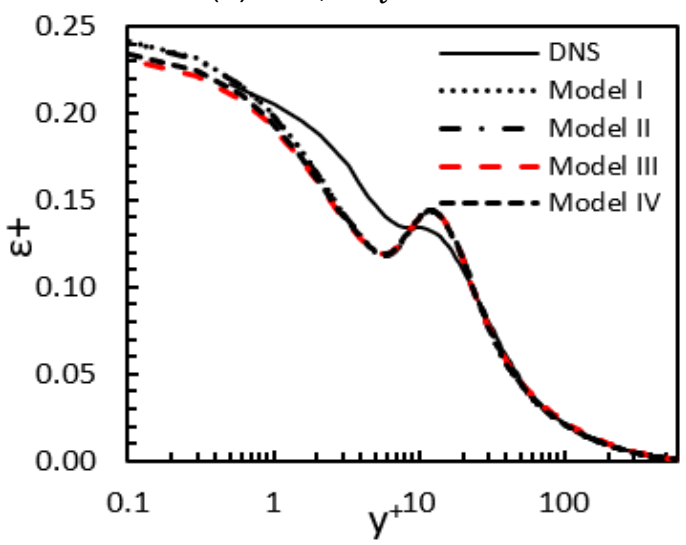

(f) $\varepsilon^{+} ; R e_{\tau}=590$

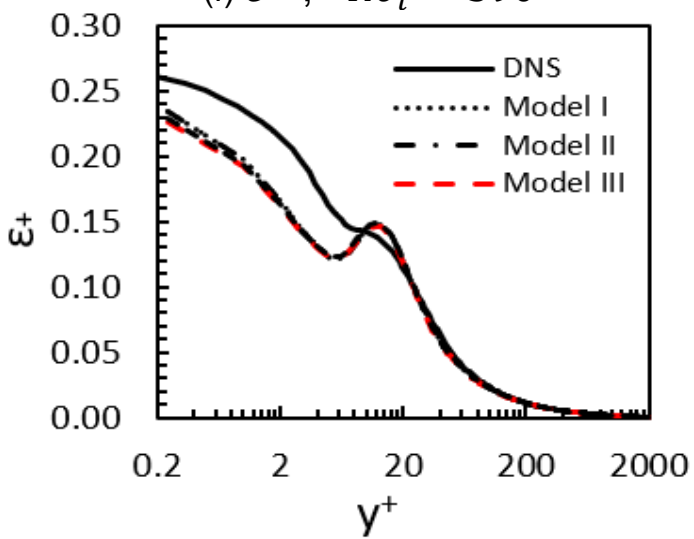

(g) $\varepsilon^{+} ; R e_{\tau}=2000$

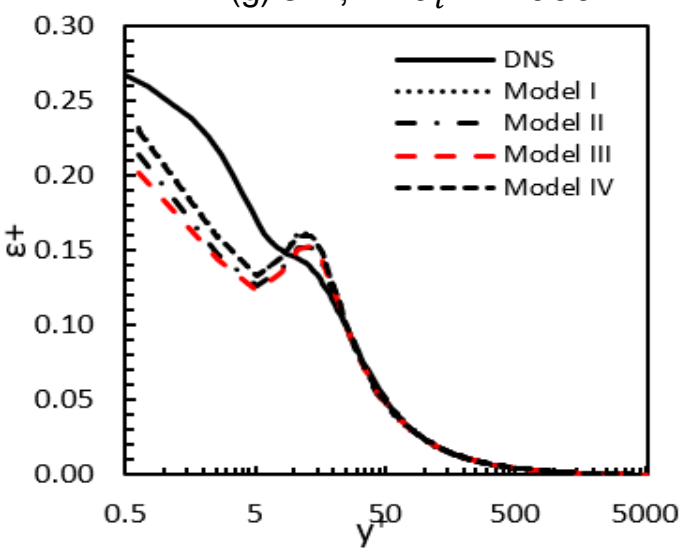

(e) $\varepsilon^{+} ; R e_{\tau}=5200$

Figure 4.18 - Comparison of turbulent kinetic energy and its dissipation with DNS data. 
Figure 4.19 shows the results for the Reynolds Stress profile for all values of Reynolds number presented in this subsection. It is possible to observe that the all components have the same pattern near the wall $\left(y^{+}<90\right)$ while the outer region varies depending on the Reynolds number.
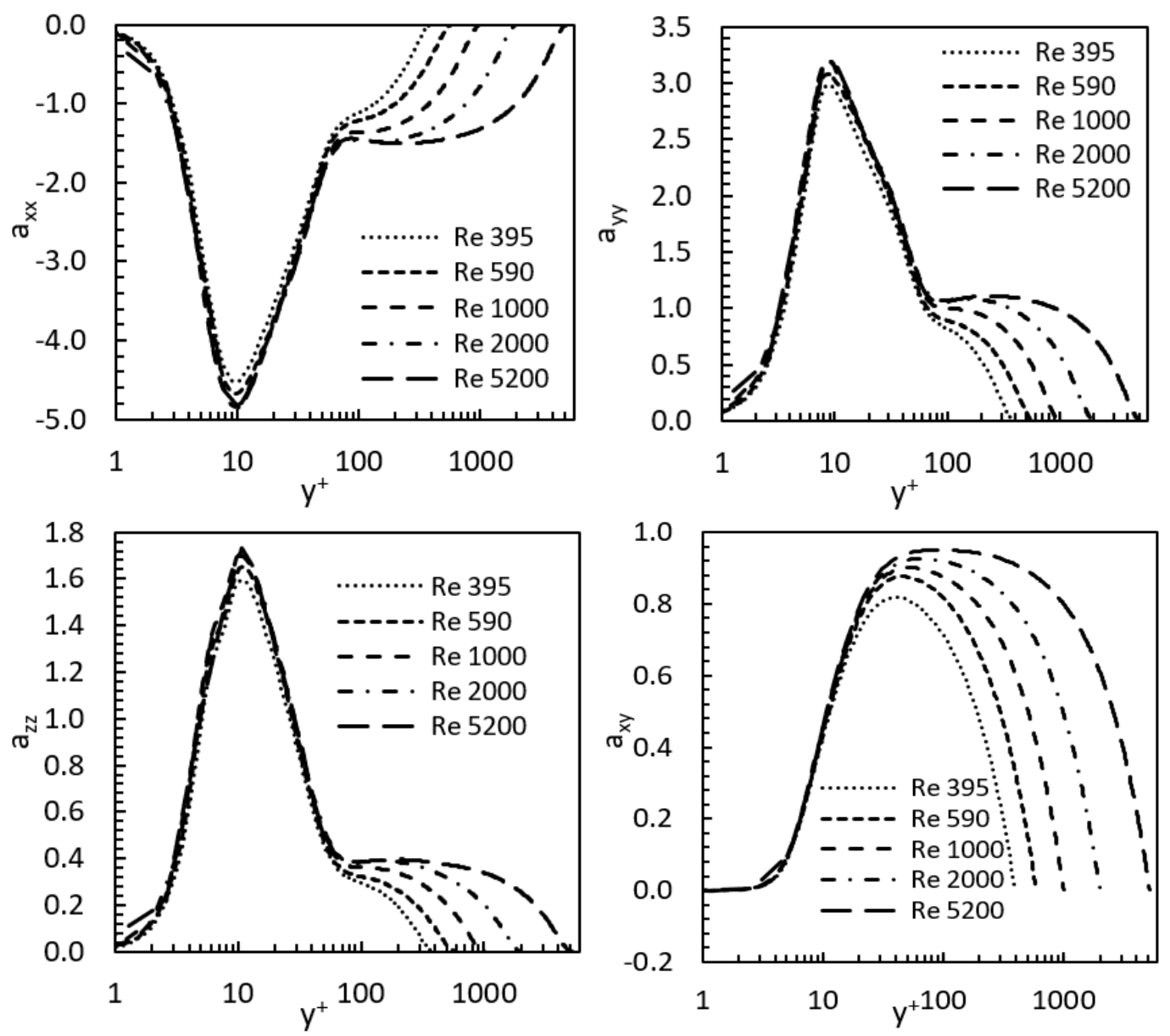

FiguRE 4.19 - MOdEL III REYNOLdS StRESS FOR DIFFERENT REYNOLDS NUMBER

\subsection{K-Y Models' Results}

In this subsection the results obtained with the $\kappa-\gamma$ models for $R e_{\tau}=1000$ are presented. The same mesh and properties were specified to simulate the flow as in the $\kappa-\varepsilon R e_{\tau}=1000$ case.

Figures 4.20 through 4.23 present a comparison of the results obtained with the two types of nondimensionalization of the models' coefficients, i.e, employing $\kappa-\varepsilon$ and $\kappa-\gamma$. These results are also compared with the DNS data. All $\kappa-\gamma$ and $\kappa-\varepsilon$ models follow the same trend as the a priori analysis. Model II provides the same $a_{x x}$ and $a_{y y}$ components. Model III is the one which can best predict the Reynolds 
tensor. Model IV under estimates $a_{x x}$ and super estimates $a_{y y}$ and cannot evaluate $a_{z z}$

Starting the discussion with the velocity field shown in Fig. 4.20, it can be seen that both families of models accurately and similarly predict this quantity (the maximum difference between the two curves results is less than $2 \%$ ). At the same figure, the shear stress Reynolds component $a_{x y}$ is presented. The agreement of both models' families is also very good and equivalent. There is a small difference between the predictions of $\kappa-\gamma$ model for $y^{+}$between 2 and 10 , when a perfect agreement with DNS data can be seen, while $\kappa-\varepsilon$ models detaches from the DNS. On the other hand, the $\kappa-\varepsilon$ models, performs better for $y^{+}$between 10 and 20 . The agreement of the shear component has a direct effect on the prediction of the mean axial velocity, with equivalent behavior. Overall, both models accurately predict the $a_{x y}$ component: for Model I for example both nondimensionalization presented less than $2.1 \%$ of average error and less than 0.043 absolute error.

The great advantage of $\kappa-\gamma$ nondimensionalization is evident when one is interested in the normal components of the Reynolds stress tensor. The comparison of the models prediction with the two types of nondimensionalization is shown in Figs. 4.21 and 4.22. As already discussed, Model I is unable to predict the normal components, therefore, at Fig. 4.21, the results for $a_{x x}$ and $a_{y y}$ are shown for Models II, III and IV. Since Model IV is unable to predict the $a_{z z}$ component, Fig. 4.22 only presents the results obtained with Models II and III. For all normal Reynolds stress components $\kappa-\gamma$ models prediction is superior to $\kappa-\varepsilon$ counterpart. It is also possible to notice that not only the resulting profiles are smoother but the shift problem discussed in Appendix $\mathrm{D}$, due to $\kappa$ and especially $\varepsilon$ influence, disappears for $\kappa-\gamma$ models.

The superiority behavior of the $\kappa-\gamma$ models can be attributed to the prediction of the dissipation of the turbulent kinetic energy $\varepsilon$. With $\kappa-\gamma$ nondimensionalization, the influence of this variable is indirect, since it only affects the $\kappa$ prediction, though the destruction term in the $\kappa$ equation, therefore avoiding an important and direct affect in the calculation of the Reynolds stress components.

Finally, the prediction of both $\kappa$ and $\varepsilon$ is shown in Fig. 4.23. The $\kappa$ field is better predicted by $\kappa-\gamma$ models which matches the DNS data for almost the whole domain. The region of $y^{+}$between 5 and 30 is where the prediction field detaches 
from DNS data. For this region, both sets of models over estimate this quantity. However, $\kappa-\gamma$ models are still more accurate than its $\kappa-\varepsilon$ counterpart.

For the $\varepsilon$ variable, no major changes are observed away from the wall, where the profiles approach zero in every case. Near the wall, no major difference is observed for this field when comparing only the models in each respective set. When comparing models of different sets, however, the profiles are slightly different. Near the wall, the dissipation rate is higher for the $\kappa-\gamma$ nondimensionalization relatively to $\kappa-\varepsilon$. The boundary $\varepsilon$ is also better predicted by $\kappa-\gamma$ models in the case of Models III and IV.

The nondimensionalization of the models' coefficient with $\kappa-\gamma$ has the following advantages: (1) The influence on the flow prediction of the model to determine $\varepsilon$ is reduced. (2) Better results for the normal components were obtained. 

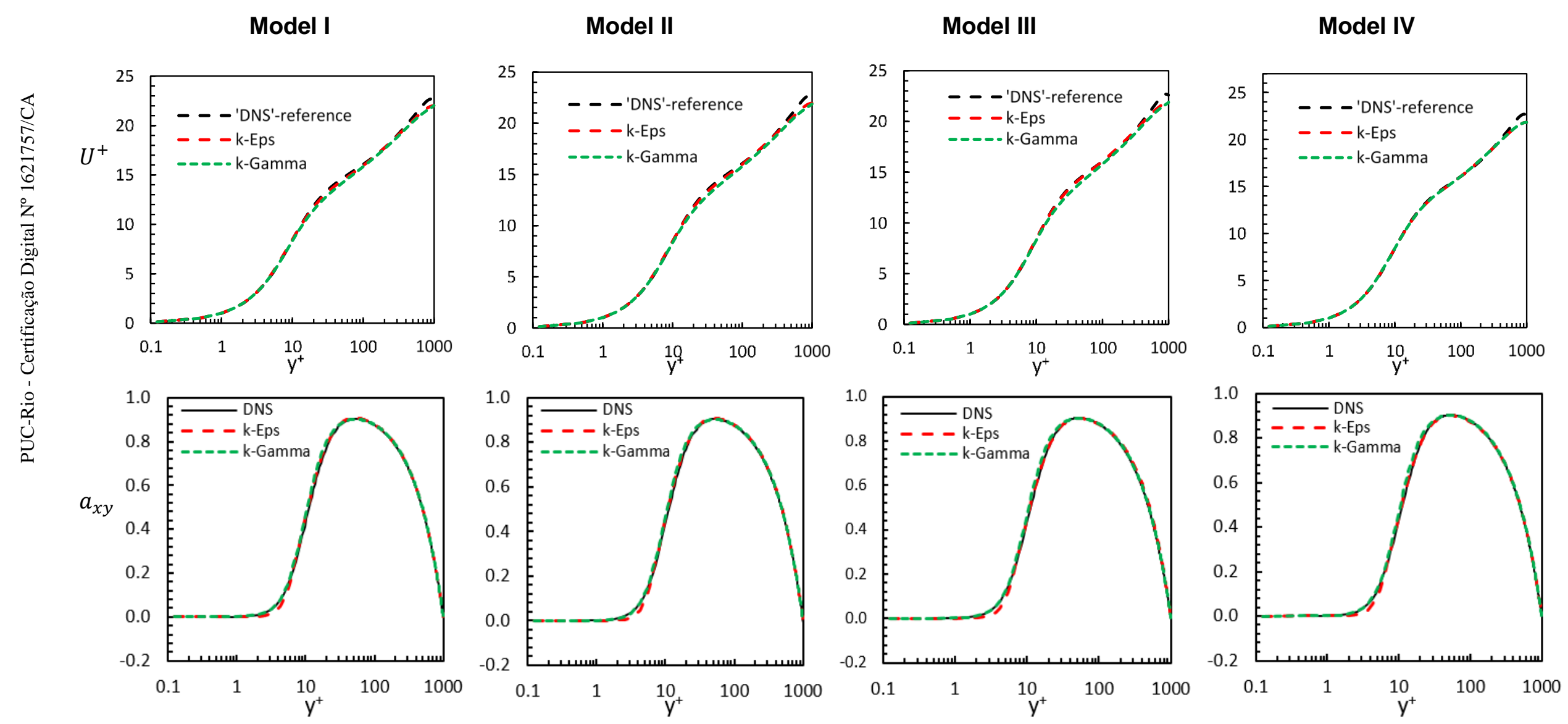

Figure 4.20 - Comparison of models $\boldsymbol{\kappa}-\boldsymbol{\gamma}$ and $\boldsymbol{\kappa}-\boldsymbol{\varepsilon}$ models for the mean axial velocity and shear Reynolds stress component. 

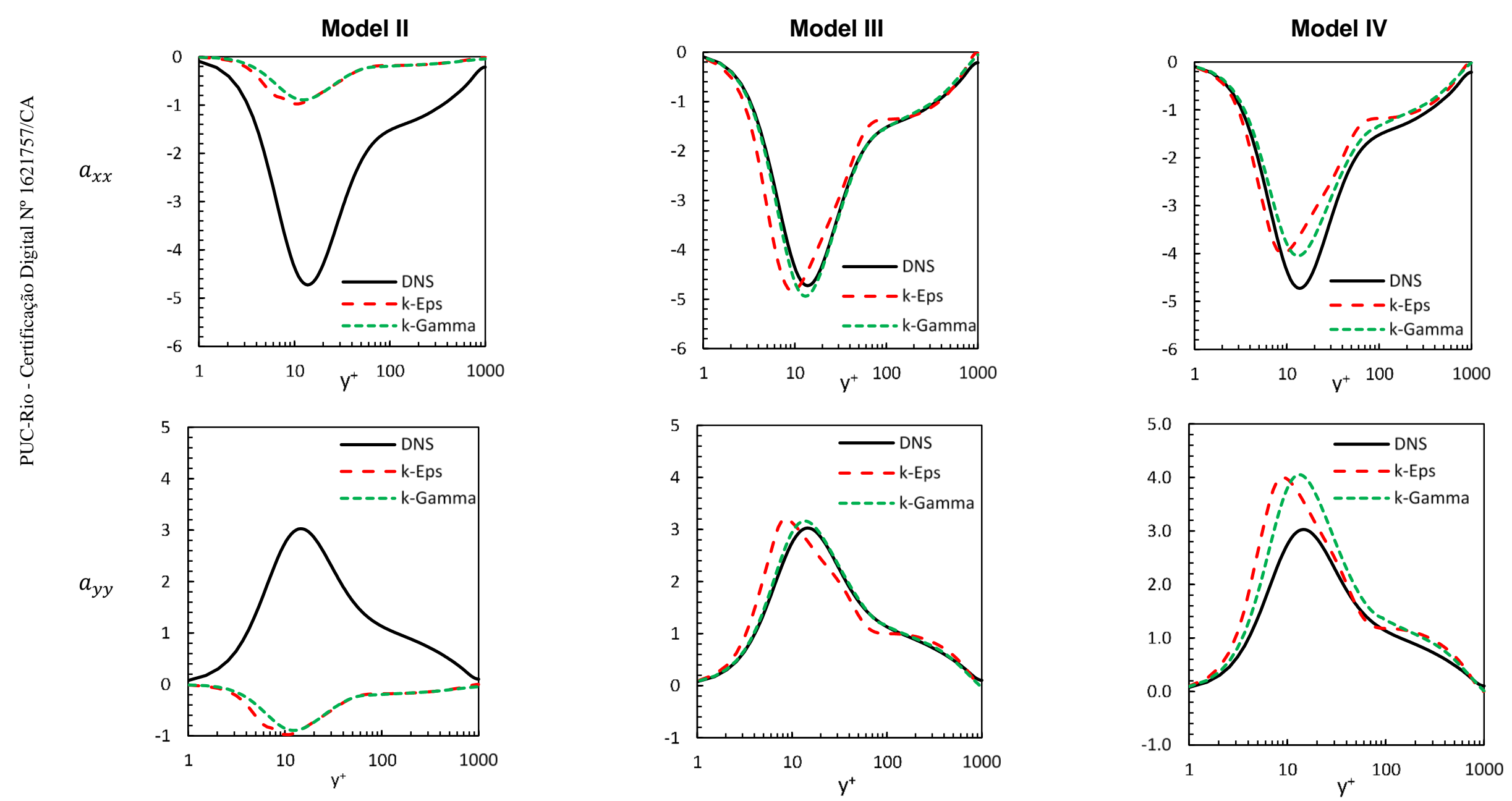

Figure 4.21 - Comparison of models $\mathrm{k}-\mathrm{\gamma}$ and $\mathrm{k}-\varepsilon$ models for the normal Reynolds stress component, $a_{x x}$ and $a_{y y}$ 
Model II

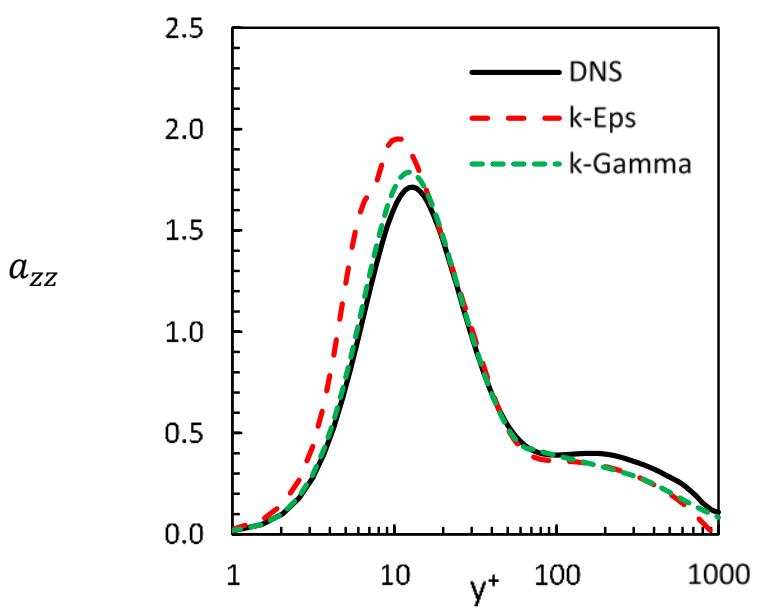

Model III

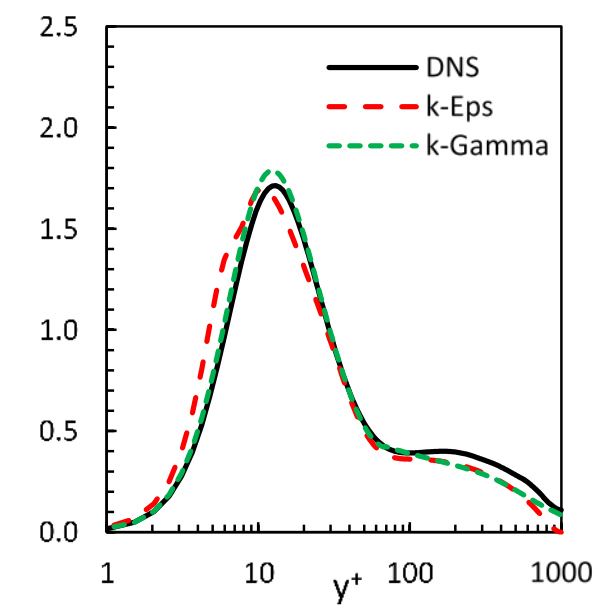

Figure 4.22 - Comparison of models $\mathrm{k}-\mathrm{\gamma}$ and $\mathrm{k}-\varepsilon$ models for the normal Reynolds stress component, $a_{z z}$ 

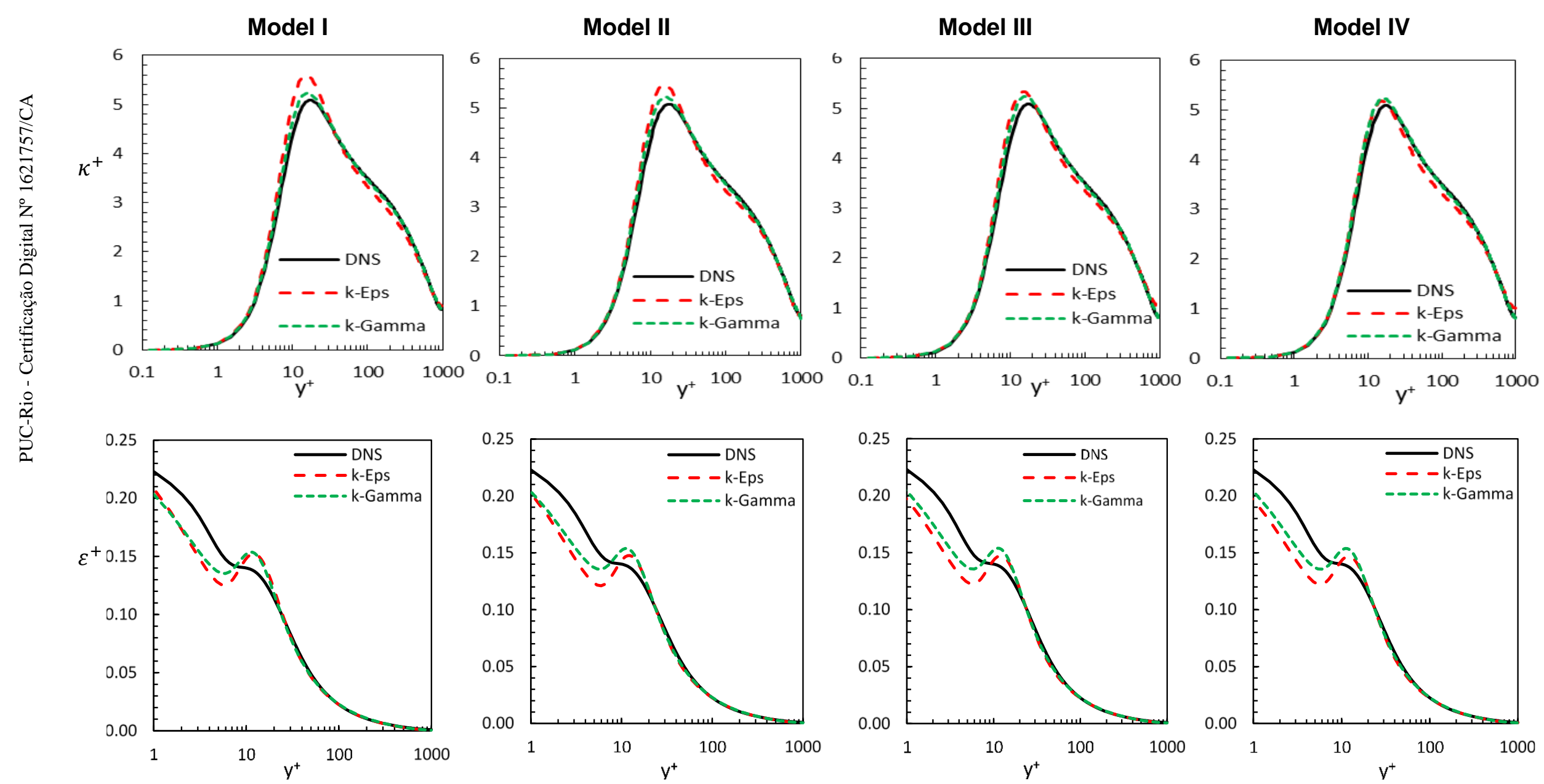

Figure 4.23 - Comparison of models $k-y$ and $k-\varepsilon$ models for the turbulent kinetic energy and its dissipation. 


\section{Conclusion}

The main objective of this work was to prove that non-linear eddy viscosity models, especially when using the non-persistence of strain $(\mathrm{P})$ tensor, quadratic of rate of strain $\left(D^{2}\right)$ and rate of strain's intensity $(\gamma)$, can be used in simulations to reduce the error associated with the Boussinesq hypothesis. By doing that, better predictions of the turbulence, majorly with respect to the Reynolds Stress tensor, were expected.

To do that, in the present work, existing linear and non-linear models were assessed by simulating a turbulent, incompressible steady state flow in a channel, for friction Reynolds number ranging from 395 to 5200. Low Reynolds Linear models were compared among themselves in order to choose the one that provides the best $\kappa$ and $\varepsilon$ fields. These fields are required to build the final non-linear model coefficients and, for this reason, the Rodi Mansour model was chosen as the most promising.

Four non-Linear $\kappa-\varepsilon$ models were, simulated using the Rodi Mansour model as a provider of the $\kappa$ and $\varepsilon$ fields. The results from these simulations agree with the trends suggested from the a priori analysis, and even the normal components of the Reynolds Stress Tensor could be accurately captured. Among the non-linear $\kappa-\varepsilon$, the one that adds both contributions of the quadratic of the rate of strain and the non-persistence of strain is the one that best matched the DNS data, despite shifts in the maximum values being observed in the solution when compared to the DNS data. A careful analysis was performed and these problems were attributed to inaccurate $\kappa$ and, specially, $\varepsilon$ fields.

The non-linear models were simulated for cases with different Reynolds number. The results shown that the model is general enough to be valid for low and high Reynolds Numbers.

Model III was compared to the Lien cubic model (Lien et al., 1991) already implemented in OpenFoam. Model III showed a better performance in the prediction of the components of the Reynolds stress Tensor, including the normal components which are under estimated by the Lien cubic model. 
Four $\kappa-\gamma$ models were numerically solved for the same geometry and Reynolds Number than the $\kappa-\varepsilon$ ones. All resulting profiles were compared to their $\kappa-\varepsilon$ counterpart. No major difference could be observed for the velocity, $a_{x y}$ and dissipation fields. However, better results were obtained for the normal components of the Reynolds Tensor when compared to the DNS data. The models based on $\kappa-\varepsilon$ showed a shift in relation to $\mathrm{y}^{+}$for the Reynolds stress components. This shifting problem is significantly mitigated by the $\kappa-\gamma$ nondimensionalization and a better agreement with the a priori analysis was observed.

\subsection{Future Work}

Several possibilities to continue the present work can be suggested. It is desirable to evaluate the models in more complex geometries, such as a backstep, a fully developed flow in a square duct, or a jet.

Rodi Mansour Modified model (Michelassi et al. 1993) has been proposed for a channel flow, therefore, it relies only on the y direction as the normal direction. This was sufficient for the present work, however, for other geometries, the model should be extended to a more general formulation, i.e., geometry independent.

Despite being the best linear model, the Rodi Mansour Modified model was not able to fully match the DNS profile. Another possible way of improvement, therefore, is to enhance the model's predictability of the $\varepsilon$ field by changing its damping functions. These functions' definitions are also a major challenge for complex flows, specially for flows with huge adverse pressure gradient and separation.

The main goal of the $\kappa-\gamma$ models was to provide smooth damping functions. Further, the reduced dependence on the dissipation $\varepsilon$ is a great benefit of this formulation. However, the particular model implemented here for the $\kappa-\gamma$ models, does not completely eliminate the $\varepsilon$ field. Thus, one possible future development is to eliminate completely $\varepsilon$ from the model, introducing a new model for the destruction of the turbulent kinetic energy on its equation. This would not only make the solution to run much faster but also prevent coupling convergence problems, as one transport equation would be excluded.

In the $\kappa-\gamma$ model, the eddy viscosity and the other non-linear terms are expressed divided by the rate of strain tensor intensity $(\gamma)$. This can be a problem 
for flows with zero velocity gradients (such as the channel flow at the symmetry plane). When the velocity gradient is close to zero, these quantities tend to infinity which makes the solution convergence much harder to attain. Therefore, improvements in the converging techniques can also be the focus of future work.

The $\kappa-\gamma$ models still requires to have their universality assessed for a broad range of Reynolds numbers, as done for the $\kappa-\varepsilon$ models. However, the coefficients calibration of the $\kappa-\gamma$ models depend on the friction Reynolds Number, and they must be first determined.

With this work, and the works to come, it is believed that new tools to predict the turbulence phenomena, such as the non-persistence of strain tensor and the rate of strain intensity, were proven valuable. The user of these models is expected to have better prediction of the turbulent flow in simple and, in the future, complex geometries than conventional eddy viscosity models. 


\section{Bibliography}

1. Abe, K.; Jang, Y.-J.; Leschziner, M. An investigation of wall-anisotropy expral Heat and Fluid Flow, 2003. p. 181-198.

2. Alves, F. V.;Thompson, R. L.; Sampaio, L. B. O Uso de Base de Dados DNS para Construção de Modelos RANS Utilizando Decomposições Tensoriais e Coeficientes de Bases Normalizadas. Rio de Janeiro, 2014 Monografia (Mestrado em Engenharia Mecânica) -Universidade Federal do Rio de Janeiro.

3. Baldwin, B.; Lomax, H. Thin Layer Approximation and Algebraic Model for Separated Turbulent Flows. AIAA, 1978. p.78-257.

4. Bernardini, M.; Pirozolli, S.; Orlandi, P. Velocity Statistics in Turbulent Channel Flow up to Ret=4000. Journal of Fluid Mechanics, 2014. p. 171-191.

5. Bilger, R. W. Turbulent Mixing and Reaction. 11th Australian Fluid Mechanics Conference. 1992

6. Bradshaw, P.; Huang, G. P. The Wall of Law in Turbulent Flows. Osborne Reynolds Centenary, 1995.

7. Gloor, M.; Bühler, S.; Kleiser, L. Transition to turbulence and noise radiation in heated coaxial jet flows. Physics of Fluids, 2016.

8. Harlow, F. H., \& Nakayama, P. I. Transport of Turbulence Energy Decay Rate. California: Los Alamos Science Laboratory, 1968.

9. He, S.; Seddinghi, M. Transition of transient channel flow after a change in Reynolds number. Journal of Fluid Mechanics, 2015. p. 395-427.

10. Hosseinverdi, S.; Boroomand, M. Prediction of Laminar-Turbulent Transitional Flow over Single and Two-Element Airfoils. 40th Fluid Dynamics Conference and Exhibit, 2010

11. Jones, W.; Launder, B. The prediction of laminarization with a two-equation model of turbulence. International Journal of Heat and Transfer, 1972. p.301-314.

12. Lam, C.; Bremhorst, K. Modified Form of $\mathbf{k - \varepsilon}$ for Predicting Wall Turbulence. Journal of Fluids Engineering, 103, 1981. p.456-460.

13. Launder, B.; Pridding, C.; Sharma, B. The Calculation of Turbulent Boundary Layers on Spinning and Curved Surfaces. Journal of Fluids Engineering, 1977. p. 99- 231.

14. Lee, M.; Moser, R. Direct Numerical Simulations of Turbulent Channel Flow up to Ret=5200. Journal of Turbulence, 2015. p. 395-415. 
15. Lien, F.; Leschziner, M. A Pressure-Velocity Solution Strategy for Compressible Flow and Its Application to Shock/Boundary-Layer Interaction Using SecondMoment Turbulence Closure. Journal of Fluids Engineering, 1993. P.717-725.

16. Lien, F.; Chen, W.; Leschziner, M. Low-Reynolds-Number Eddy-Viscosity Modelling Based on Non-Linear Stress-Strain/Vorticity Relations. Engineering Turbulence Modelling and Experiments, 1991. p.91-100.

17. Menter, F. Two-equation eddy-viscosity turbulence models for engineering applications. AIAA Journal, 1994. P.1598-1605.

18. Michael, O. J.; Dominik, O.; Kleiser, L. Stabilisation of subcritical bypass transition in the leading-edge boundary layer by suction. Journal of Turbulence, 2014

19. Michelassi, V., Rodi, W., \& Zhu, J. Testing a Low Reynolds Number k- $\varepsilon$ Turbulence Model Based on Direct Simulation Data. AIAA Journal, 1993. p.1720-1723.

20. Nieckele, A.; Thompson, R.; Mopean, G. Anisotropic Reynolds stress tensor representation in shear flows using DNS and experimental data. Journal of Turbulence, (2016). p.1-29.

21. openFOAM. Retrieved from https://openfoam.org/ Acessed in 2018, 0523.

22. Patankar, S. V. Numerical Heat Transfer and Fluid Flow. Series in Computational Methods in Mechanics and Thermal Science. 1980

23. Pope, S. B. Turbulent Flows. Cambridge University Press. 2000

24. Rodi, W.; Mansour, N. Low Reynolds number k-e modelling with the aid of direct simulation data. J. Fluid Mech, 1993. p. 509-529.

25. Schlichting, H. Boundary-Layer Theory. 1979

26. Sharath, G. S. Fully-Explicit and Self-Consistent Algebraic Reynolds Stress Model. Theoretical and Computational Fluid Dynamics. 1996

27. Shih, T.; Liou, W.; Shabbir, A.; Yang; Z.; Zhu, J. A New k-e Eddy Viscosity Model for High Reynolds Number Turbulent Flows-Model Development and Validation. Ohio: NASA. 1994

28. Smith, A.; Cebeci, T. Numerical solution of the turbulent boundary layer equations. Douglas aircraft division report. 1967

29. Tennekes, H.; Lumley, J. L. A First Course in Turbulence. The MIT Press. 1972

30. Thais, L.; Gatski, T.; Mompean, G. Some Dynamical Features of Turbulent Flow of a Viscoelastic Fluid for Reduced Drag. Journal of Turbulence, 2012. p.1-26.

31. Thompson, R. L. (2008). Some Perspectives of Dynamic History of a Material Element. International Journal of Engineering, 224-229.

32. Thompson, R. L.; Mompean, G.; Thais, L. A Methodology to Quantify the Nonlinearity of the Reynolds Stress Tensor. Journal of Turbulence, 2010. p1-27. 
33. Thompson, R. L.; Sampaio, L. B.; Alves, F. A.; Thais, L.; Mompean, G. A methodology to evaluate statistical errors in DNS data of plane channel flows. Computers and Fluids, 2016 p. 1-7.

34. Trinh, K. T. On The Critical Reynolds Number For Transition From Laminar To Turbulent Flow. Cornell University Library, 2010

35. Wilcox, D. Turbulence Modeling for CFD. San Diego: DCW Industries. 2006

36. Yakhot, V.; Thangam, S.; Gatski, T.; Orszag, S.; Speziale, C. Development of Turbulence Models for Shear Flows by a Duble Expansion Technique. Virginia: NASA. 1992

37. Zhiyin, Y. Large-Eddy Simulation, Past Present and Future. Journal of Aeronautics, 2014 


\section{Appendix A - Coefficients Adjustments for $\kappa-\varepsilon$ Models}

As mentioned in Chapter 3, Nieckele et al. (2016) recommend the Modified Rodi Mansur damping function for the linear term. They also proposed expressions for the damping functions $\left(f_{\mu 2}\right.$ and $\left.f_{\beta}\right)$ and reference coefficients: $C_{\mu}$ for the linear contribution and $C_{\mu 2}$ and $C_{\beta}$ for the non-linear terms, by fitting the DNS data. At this appendix a revaluation of the parameters proposed by Nieckele et al (2016) is performed. First, the influence of the reference $C_{\mu}$ is addressed, since Rodi Mansour (Michelassi et al. 1993) recommend a coefficient to be used with their proposed damping function and Nieckele et al. (2016), recommended another one. Then, a reevaluation of the damping functions of the linear terms $f_{\mu 2}$ and $f_{\beta}$ is also performed.

\section{A.1 Coefficient $C_{\mu}$}

In order to choose the better $C_{\mu}$ for the Rodi Mansour Modified model (RMM) into the DNS data, simulations were carried out with different $C_{\mu}$ coefficients $(0.075,0.08$ and 0.09). Figures A.1, A.2 and A.3 presents the influence of the reference coefficient in the profiles of $a_{x y}, U^{+}, \varepsilon$ and $\kappa$, respectively.

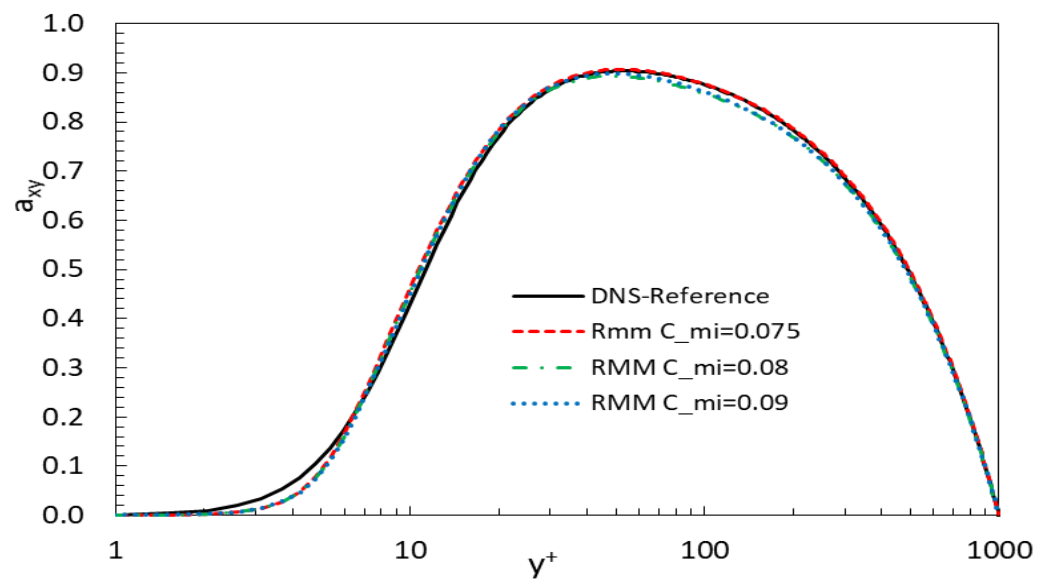

Figure A.1 - Shear Reynolds tensor component $\boldsymbol{a}_{\boldsymbol{x}}$ with Rodi Mansour Modified (RMM) damping function, with different $\boldsymbol{C}_{\boldsymbol{\mu}}$ 
Analysing the figures, it is possible to notice that the simulation with $\mathrm{C} \mu=0.075$ could reproduce better the DNS $a_{x y}, \kappa^{+}$and $\varepsilon^{+}$while no significant changes were observed for the velocity. The value of 0.075 was, therefore, chosen to be used for non-linear models' simulations. This value is, moreover, close to the one proposed by (Nieckele et al. 2016).

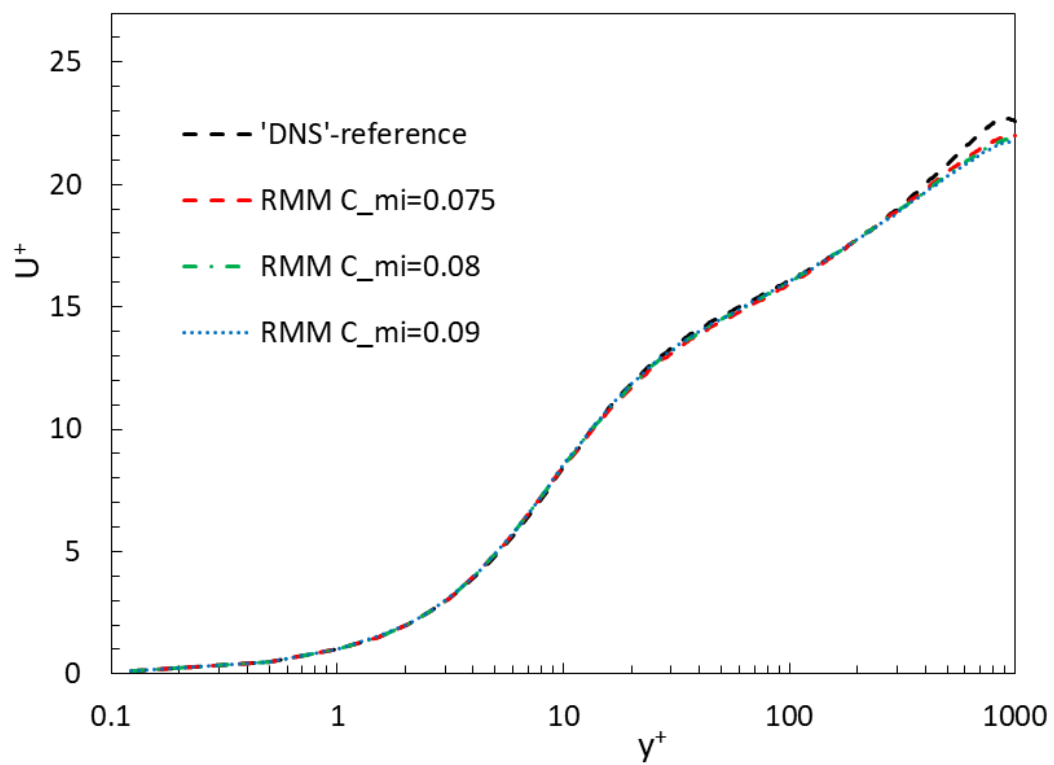

Figure A.2 Mean axial velocity with Rodi Mansour Modified (RMM) damping function, with different $\boldsymbol{C}_{\boldsymbol{\mu}}$
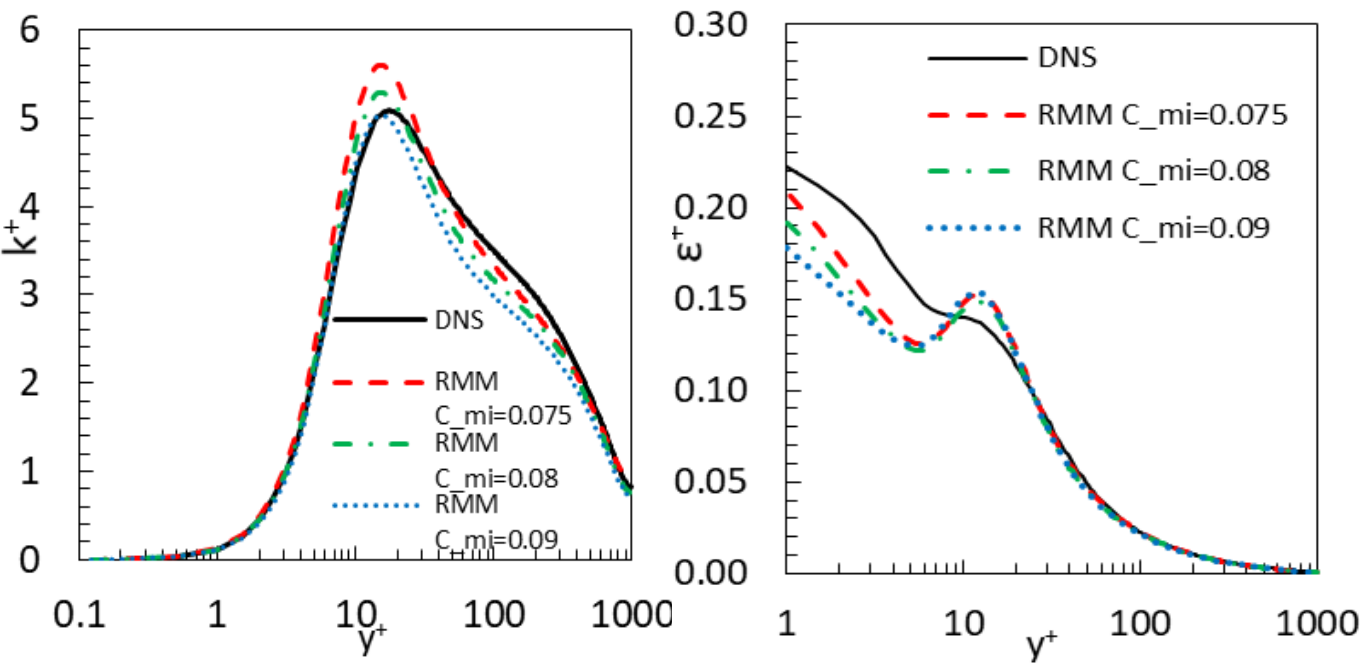

Figure A.3 - $\mathrm{K}$ and $\varepsilon$ with Rodi Mansour Modified (RMM) damping function, with different $\boldsymbol{C}_{\boldsymbol{\mu}}$ 


\section{A.2 Damping functions of non-linear terms $f_{\mu 2}$ and $f_{\beta}$}

The damping expression they recommend is shown in Eq. A.1, with $C_{\mu 2}=0.05$ and $C_{\beta}=0.05$. Figure shows the comparison with the fit with the $f_{\mu 2}$ and $f_{\beta}$ extracted from DNS.

$$
f_{\mu 2}=f_{\beta}=\frac{\tanh \left(8.5 \times 10^{-4}\left(y^{+}\right)^{2.01}\right)}{\tanh \left(1.8 \times 10^{-5}\left(y^{+}\right)^{5.35}\right)}
$$

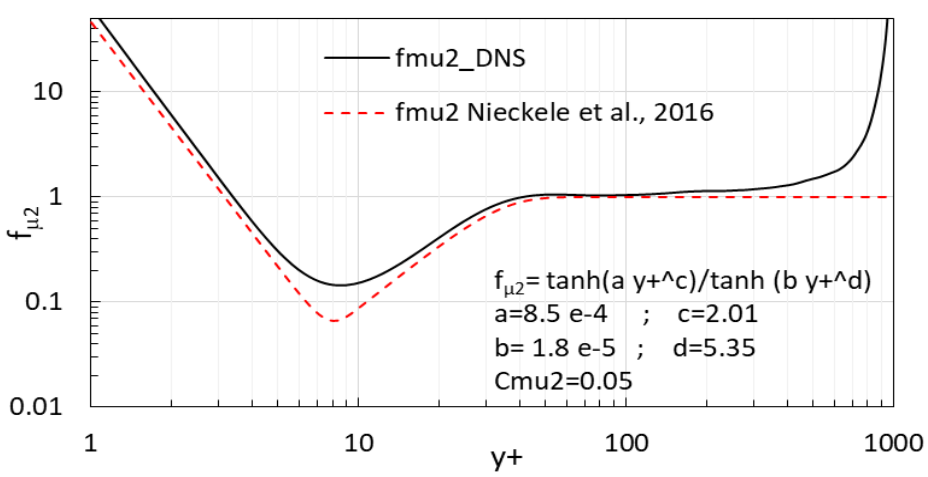

(a) Damping function of $D^{2}$ term

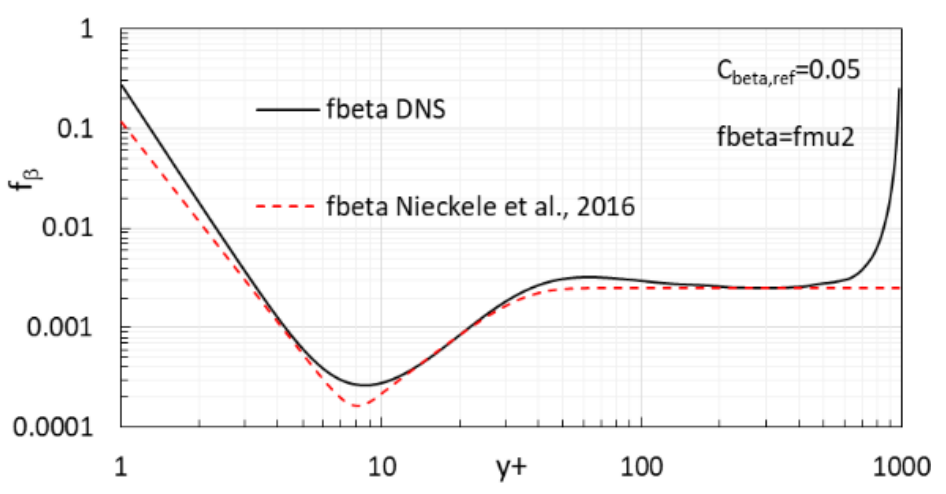

(b) Damping function of $\mathrm{P}$ term

Figure A.4 - Damping functions of the non-linear terms from Nieckele et al. (2016)

However, at the present work, the recommended fit for $f_{\mu 2}$ and $f_{\beta}$ were reevaluated using the DNS data of $\kappa, \varepsilon$ and $U$. The coefficients $C_{\mu 2}$ and $C_{\beta}$ were maintained as 0.05 . The new damping expression proposed at the present work are

$$
f_{\mu 2}=\frac{\tanh \left(6.15 \times 10^{-4}\left(y^{+}\right)^{2.135}\right)}{\tanh \left(10^{-5}\left(y^{+}\right)^{5.8}\right)}+6.1 \times 10^{-2} e^{\left(-\frac{y^{+}}{300}\right)^{2}}
$$




$$
f_{\beta}=\frac{\tanh \left(2.56 * 10^{-4}\left(y^{+}\right)^{2.305}\right)}{\tanh \left(1.3 * 10^{-5}\left(y^{+}\right)^{6.5}\right)}+1.818 * 10^{-2} e^{\left(-\frac{y^{+}}{300}\right)^{2}}+\frac{60.6}{\left(y^{+}\right)^{4}}+0.02727
$$

Figure A.5 shows a comparison of the fitting of new damping functions $f_{\mu 2}$ and $f_{\beta}$ with the damping function obtained directly from the DNS data. Although the expressions are much more complicated, a better adherence to the DNS data was obtained.

To verify the quality of the new fit of the damping coefficients, a comparison of the normal Reynolds tensor components determined with these coefficients and with the DNS velocity data are presented in Fig, A.6. The components obtained with the adjustment recommended by Nieckele et al. (2016) is also added in the figure, as well as the DNS tensor components. Looking at the plots of the Reynolds Stress tensor it's possible to observe that not only the new damping functions better fit the DNS data but also the resulting Reynolds Stress is better adjusted. Also, the new Reynolds Stress Tensor does not present an oscillation region at $y^{+} \approx 10$ anymore.

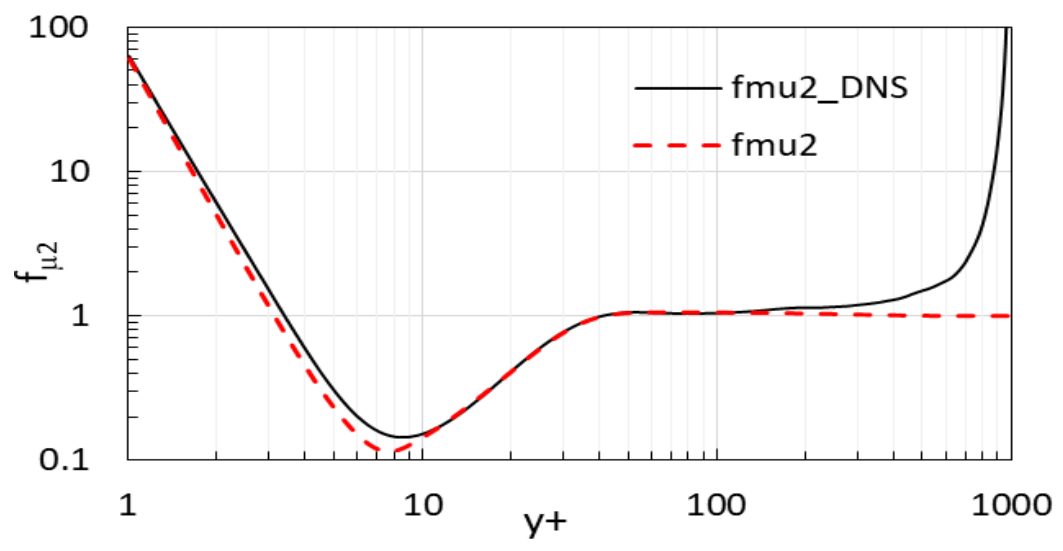

(a) Damping function of $D^{2}$ term

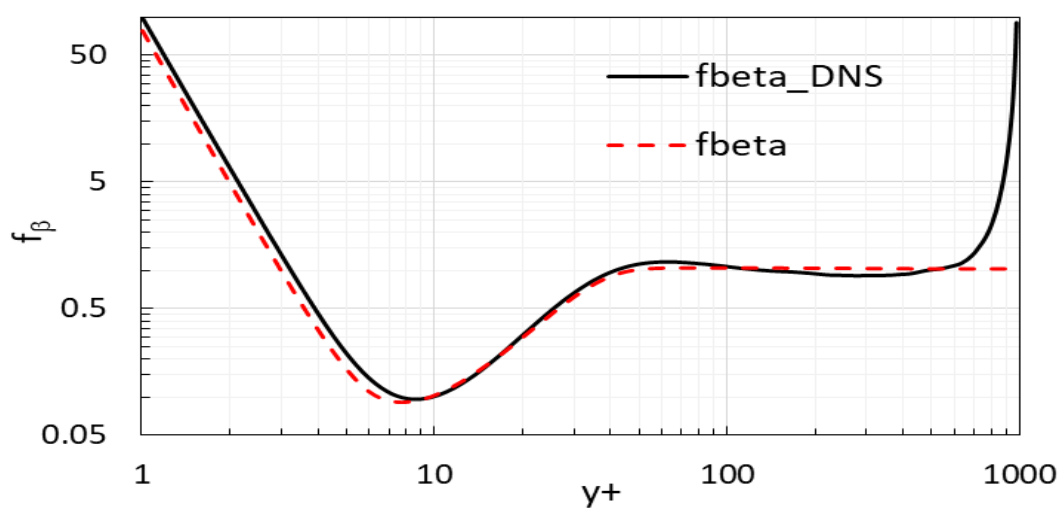

(b) Damping function of $\mathrm{P}$ term

Figure A.5 - New damping functions of the non-linear terms. 


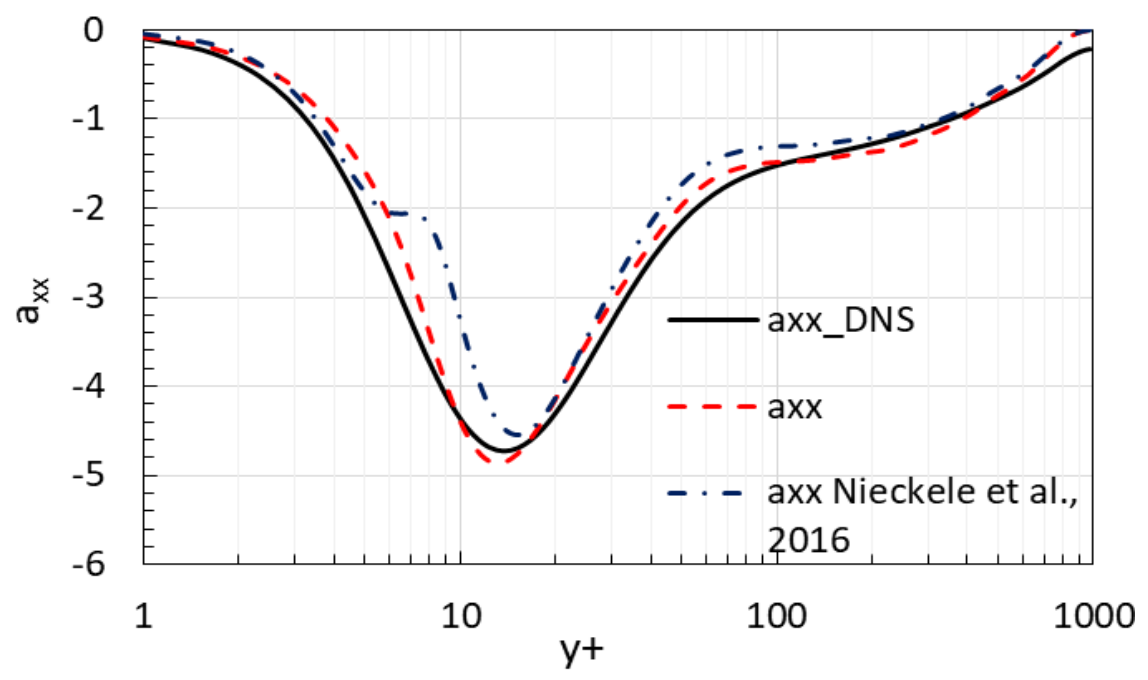

(a) $a_{x x}$ Reynolds stress component

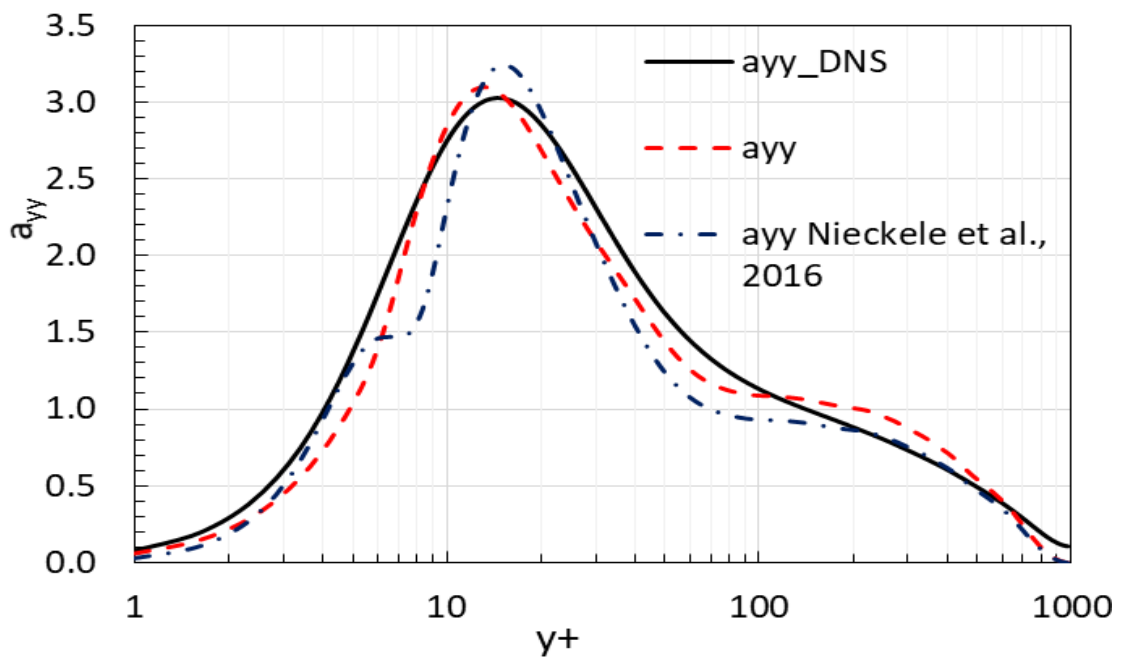

(c) $a_{y y}$ Reynolds stress component

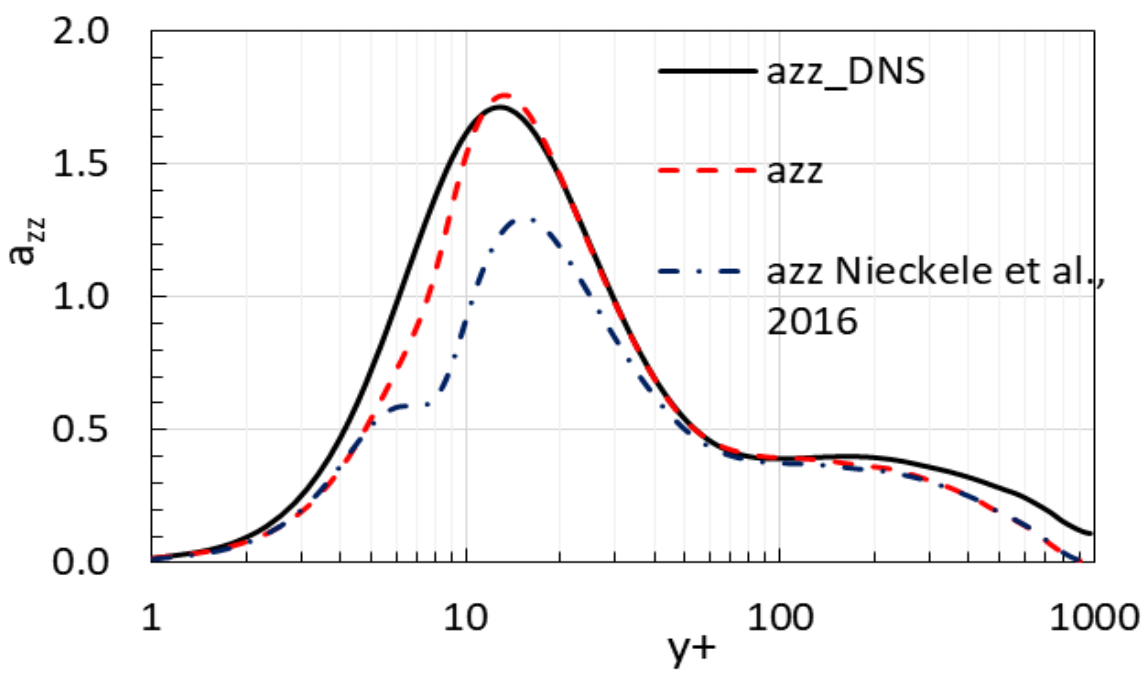

(c) $a_{z z}$ Reynolds stress component

Figure A.6 - Reynolds Stress Comparison from different damping functions 


\section{Appendix B - Coefficients Adjustments for $\kappa-\gamma$ Models}

Here, the definition of coefficients $C_{\mu-\gamma}, C_{\mu 2-\gamma}$ and $C_{\beta-\gamma}$ will be discussed. For this purpose, this section will be divided into three parts (one for each coefficient).

\section{B.1 Coefficient $C_{\mu-\gamma}$}

Figure B.1 shows the coefficient for different $\operatorname{Re}_{\tau}$ values. To calibrate this coefficient the DNS data needed to be split into three groups, in which $C_{\mu-\gamma}$ had different behaviors: $y^{+}<3,3<y^{+} \leq \hat{y}^{+}$and $\hat{y}^{+}<y^{+}$.

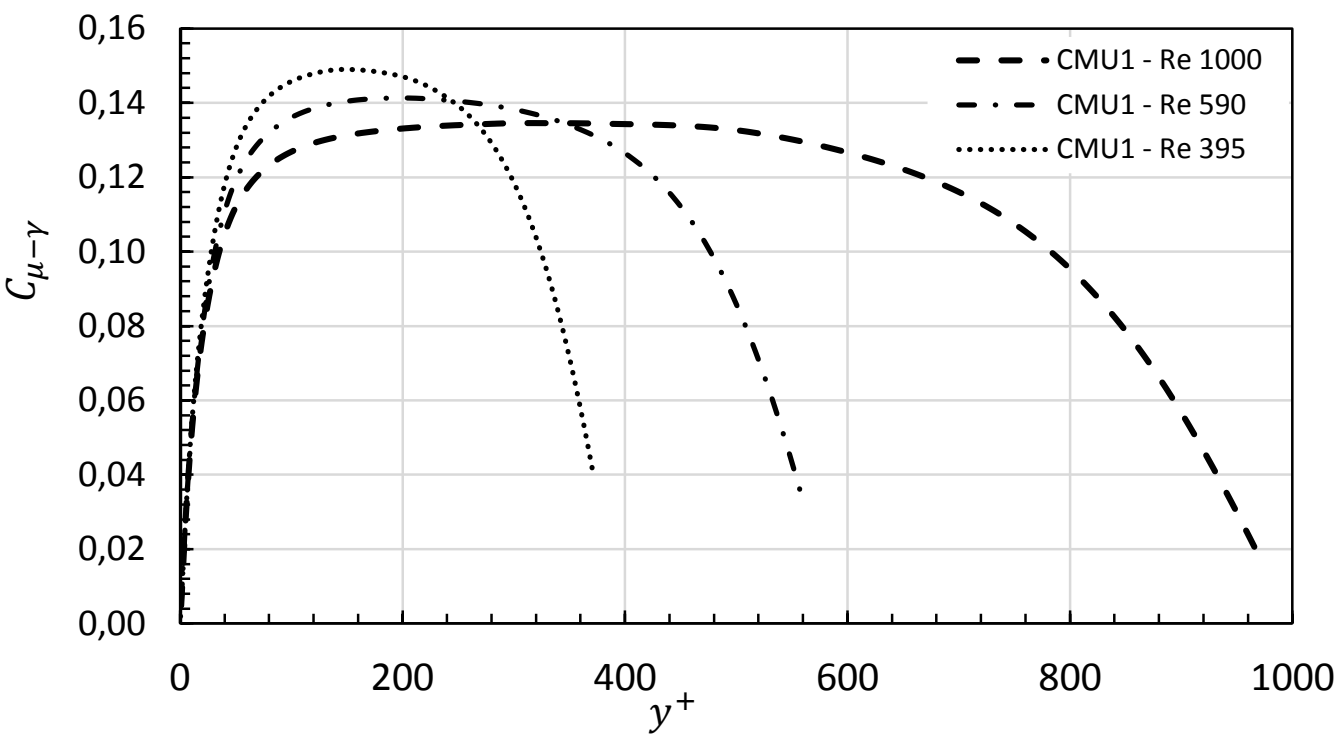

Figure B.1 $-C_{\mu-\gamma}$ from DNS for different $\boldsymbol{R e}_{\tau}: \mathbf{3 9 5 , 5 9 0 , 1 0 0 0}$

Before, proceeding to the proper discussion, first some clarifications of $\hat{y}^{+}$need to be made. $\hat{y}^{+}$is the $y^{+}$in which $C_{\mu-\gamma}$ is maximum. This value varies with the Reynolds Number as seen in figure B.1, hence, in order to obtain a generic calibration of $C_{\mu-\gamma}, \hat{y}^{+}$needs to be written as a function of Reynolds. Figure B.2 shows that $\hat{y}^{+}$can be approximated from $R e_{\tau}$ as a linear function.

The two first regions will be discussed together first, following by the third one. For this region the function that could best adjust the data is enunciated by 


$$
C_{\mu-\gamma}^{3>y^{+}>\hat{y}^{+}}=\frac{-0.007+0.0086 y^{+}}{1+0.056 y^{+}+1.5 \times 10^{-5}\left(y^{+}\right)^{2}}
$$

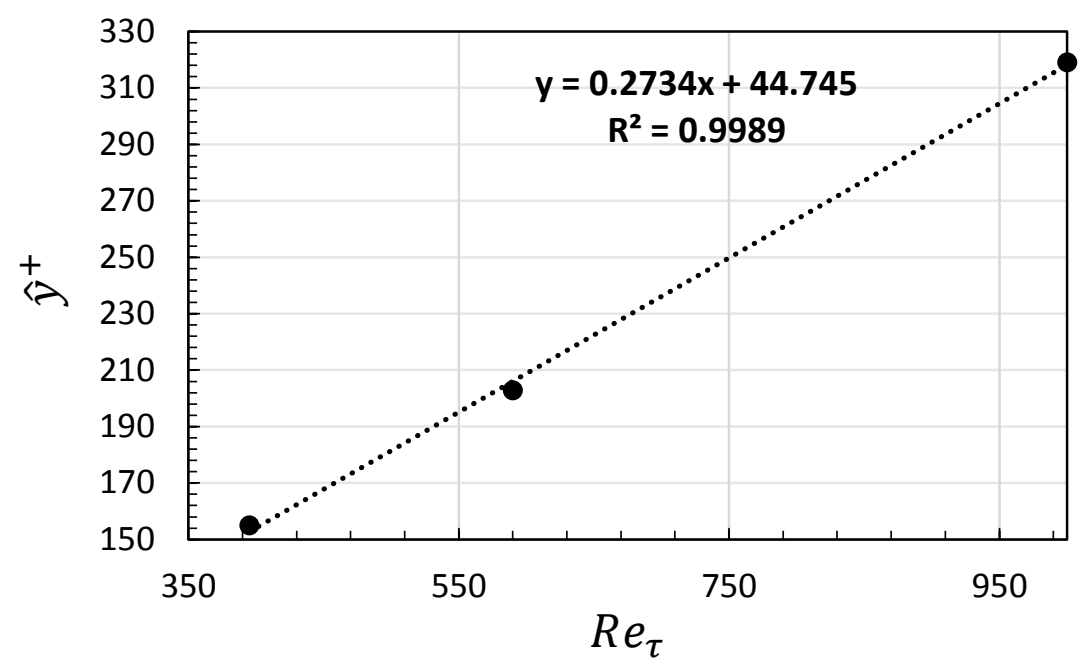

Figure B.2 - $\widehat{\boldsymbol{y}}^{+}$dependence of $\boldsymbol{R} \boldsymbol{e}_{\boldsymbol{\tau}}$

Nevertheless, for $\hat{y}^{+}$bellow 3 that function results in negative values which can cause simulation's numerical problems. In order to solve this problem another equation is set for this region: $C_{\mu-\gamma}^{y^{+}<3}=0.0054 y^{+}$. This region was only calibrated for a Reynolds of 1000. The last region $\left(\hat{y}^{+}<y^{+}\right)$is fitted by a third order polynomial function of the form of Eq. B.2. To generate a curve without spikes and steps, the first derivate and first point of the fit need to be continuous, hence,

$$
\begin{aligned}
& C_{\mu-\gamma}^{\hat{y}^{+}<y^{+}}\left(\hat{y}^{+}\right)=C_{\mu-\gamma}^{3>y^{+}>\hat{y}^{+}}\left(\hat{y}^{+}\right) \\
& \frac{\partial\left(c_{\mu-\gamma}^{\hat{y}^{+}<y^{+}}\left(\hat{y}^{+}\right)\right)}{\partial y^{+}}=\frac{\partial\left(c_{\mu-\gamma}^{3>y^{+}>\hat{y}^{+}}\left(\hat{y}^{+}\right)\right)}{\partial y^{+}}
\end{aligned}
$$

Then, the coefficients of the following equation was determined

$$
C_{\mu-\gamma}^{\hat{y}^{+}<y^{+}}=a\left(y^{+}-\hat{y}^{+}\right)^{3}+b\left(y^{+}-\hat{y}^{+}\right)^{2}+c\left(y^{+}-\hat{y}^{+}\right)+d
$$

The first restriction will result in $d=C_{\mu-\gamma}^{3>y^{+}>\hat{y}^{+}}\left(\hat{y}^{+}\right)$while the second one will produce $c=0$ since, by definition, $C_{\mu-\gamma}$ achieves its maximum value at $\hat{y}^{+}$. Only two parameters ( $\mathrm{a}$ and $\mathrm{b}$ ), therefore, requires calibration. From these parameters adjustments it was found that " $b$ " can be a constant equal to $9 \times 10^{-8}$ and "a" varies with the Reynolds' number. Table B.1 and figure B.3 shows the parameters, errors and fitting of the region. 
Table B.1 - Fitting Parameters and Errors

Reynolds Number

395

590

1000 a

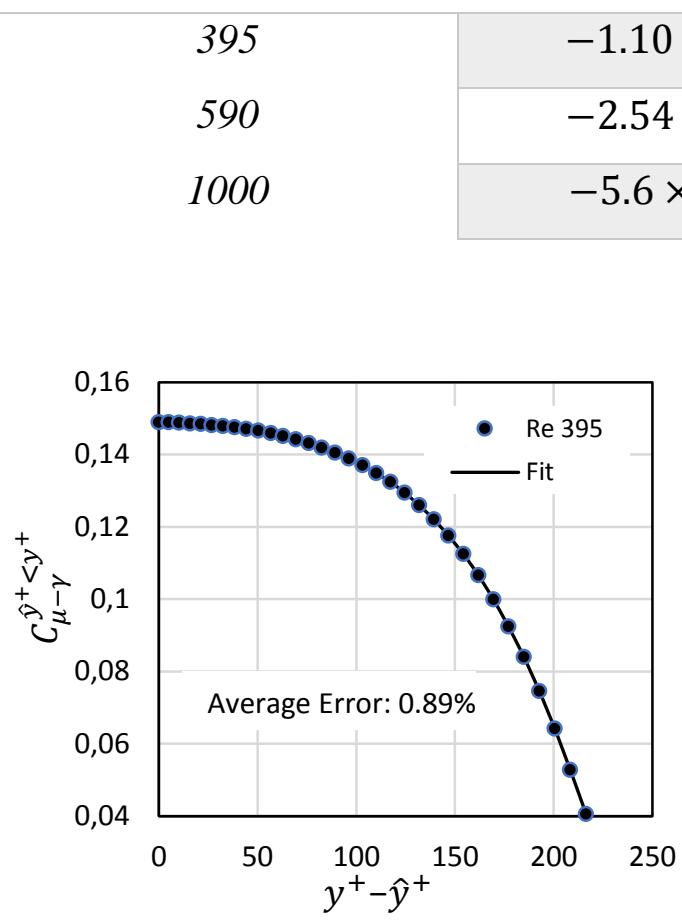

Average Error (\%)

0.89

1.72

1.49
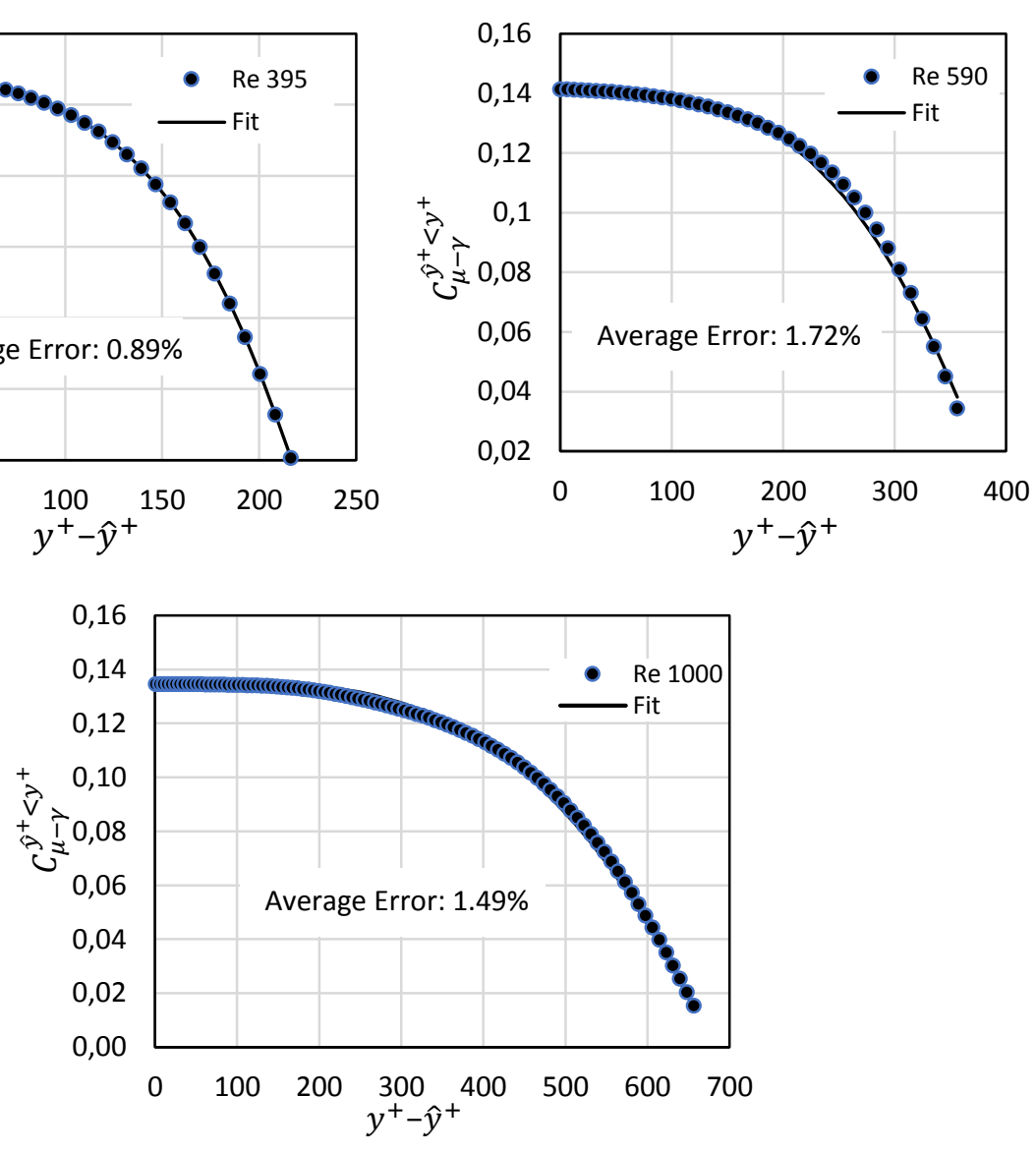

Figure B.3 - Fitting of $\boldsymbol{C}_{\boldsymbol{\mu}-\gamma}^{\hat{y}^{+}<y^{+}}$at different Reynolds Numbers

To further generalize this region's calibration, it is possible to write the "a" parameter as a $\log x \log$ function of the Reynolds Number. Figure B.4 shows the mentioned dependence as well as the respective fitted equation.

Figure B.5 exhibits the comparison of the fitting of all integrated regions with the DNS data. Although the average error is $5.25 \%$, the generated curve can reproduce correctly the behavior of the parameter. The high value of the average error is attributed to the closest region of the wall where $\boldsymbol{C}_{\boldsymbol{\mu}-\boldsymbol{\gamma}}$ has lower values and, thus, high relative errors. This is corroborated by the low maximum absolute error: 0.0162 


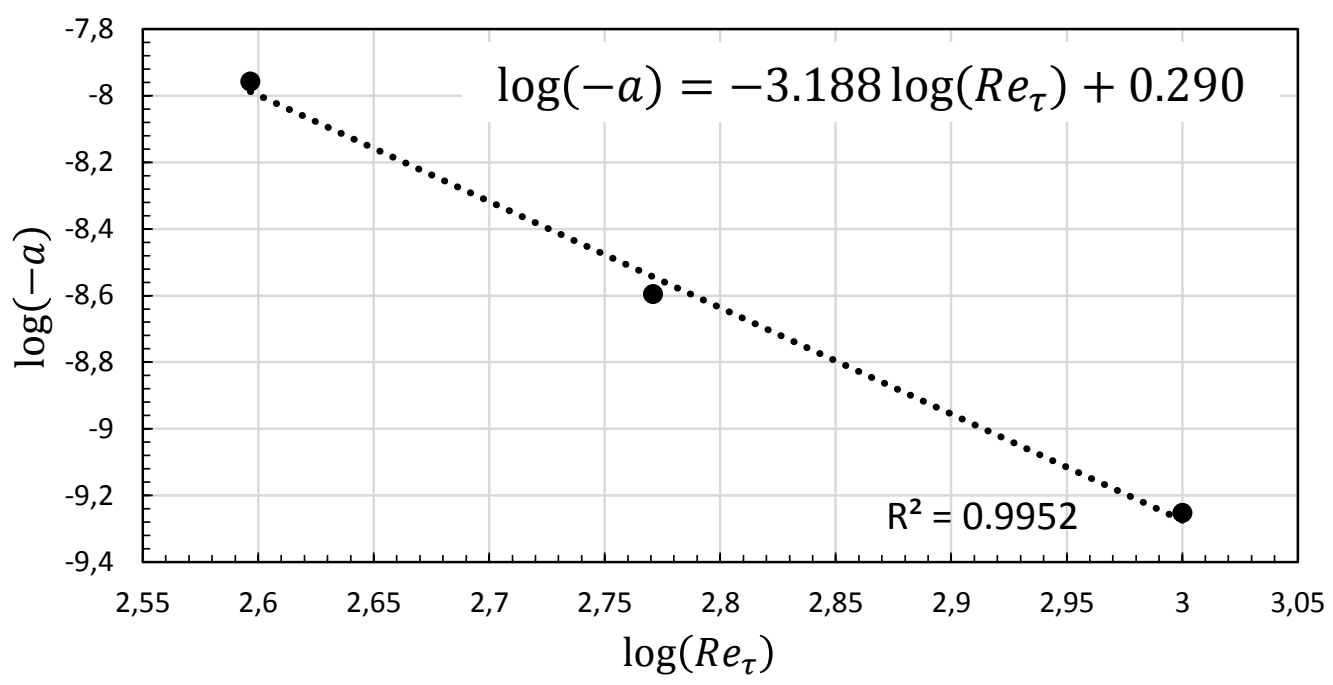

Figure B.4 - "a" parameter as a function of the Reynolds number

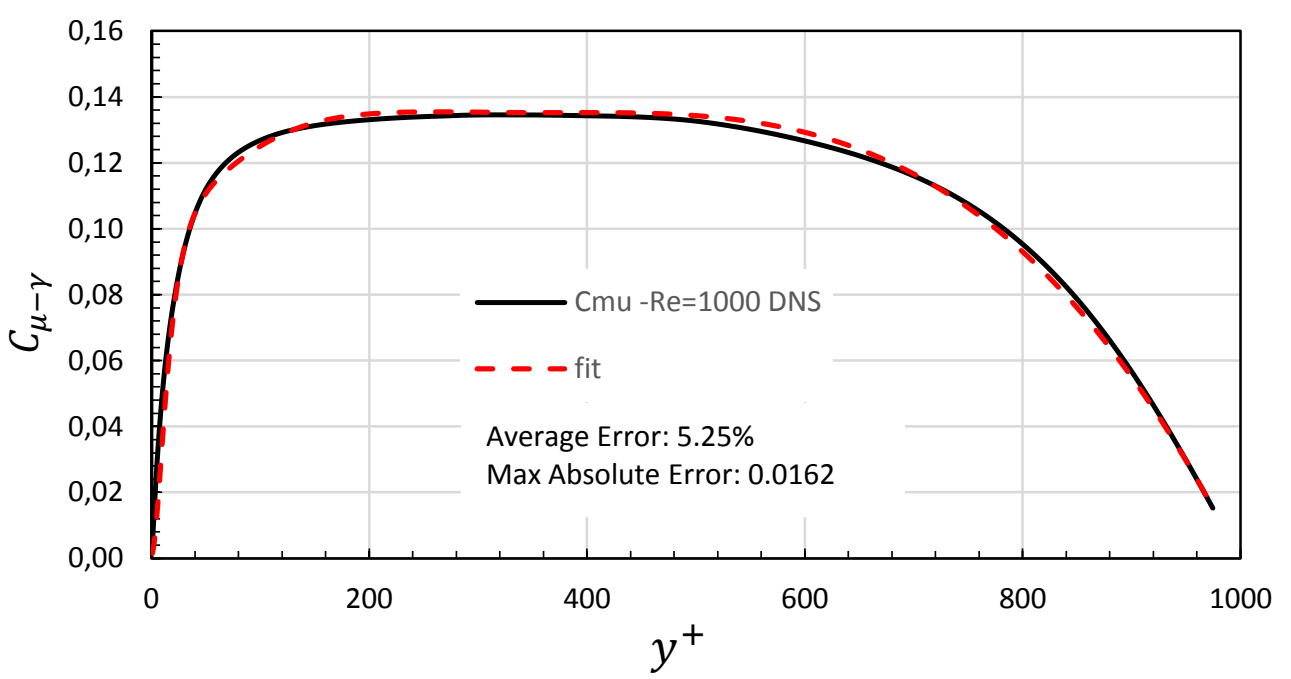

Figure B.5 $-\boldsymbol{C}_{\boldsymbol{\mu - \gamma}}$ all regions integrated and compared with the dns data

\section{B.2 Coefficient $C_{\mu 2-\gamma}$}

The $C_{\mu 2-\gamma}$ coefficient was calibrated using only the DNS $R e_{\tau}=1000$ database as a reference, hence, it is not generalized to other Reynolds values. The major area of variation of this coefficient is located between $y^{+}=1$ and $y^{+}=69$. For this area the coefficient can be represented by Eq. 3.51.

To simplify the curve fitting, the other regions $\left(y^{+}<1\right.$ and $\left.y^{+}>69\right)$ the coefficient is considered to be constant. Moreover, no discontinuations are allowed in the curve fitting. To test if this approach would not introduce modeling errors, the $\frac{\kappa}{\gamma^{2}} C_{\mu 2-\gamma} D^{2}$ term is plotted in figure Figure B.B.6. In this figure, $\kappa, \gamma$ and $D^{2}$ are 
taken from the DNS data. The difference between the curves is that $D^{2}$ term_DNS is the following term with $C_{\mu 2-\gamma}$ from the DNS while $D^{2}$ term_fit is the same term with the coefficient from the curve fitting.
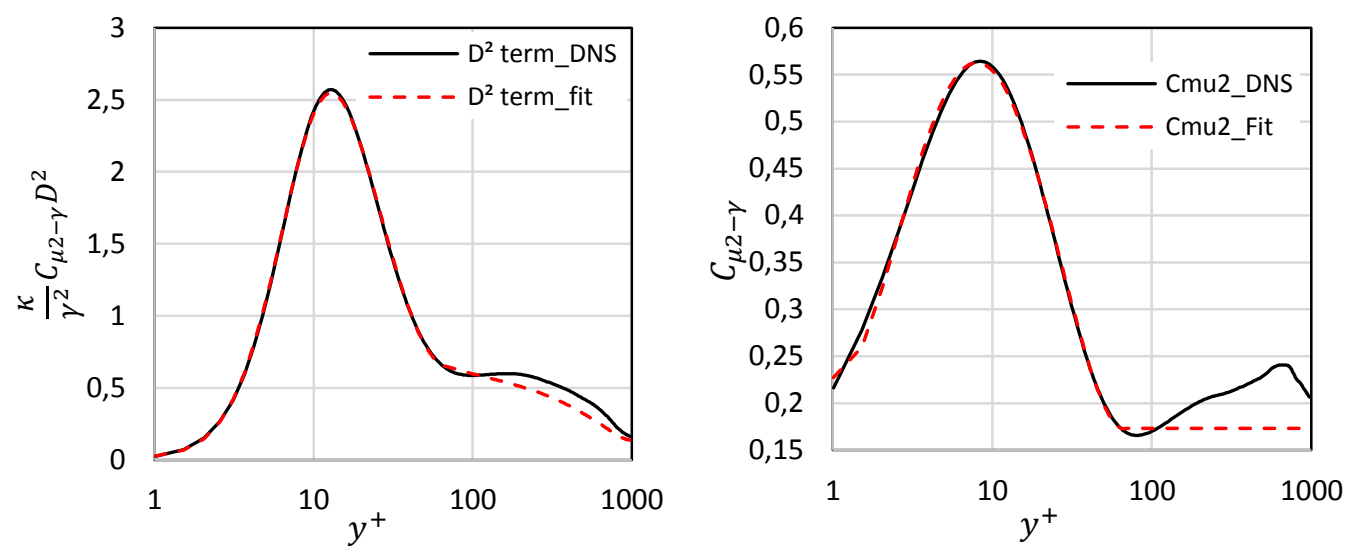

Figure B.6 - Coefficient and $\mathrm{D}^{2}$ term comparison using DNS data

As observed from the plots, although $C_{\mu 2-\gamma}$ is not correctly represented for $y^{+}$greater than 69 , there is no major effect in the $D^{2}$ term that would justify an increase in the complexity of the curve fitting. The coefficient value in the $y^{+}>$ 69 area should, therefore, be maintained constant.

\section{B.3 Coefficient $C_{\beta-\gamma}$}

The $C_{\beta-\gamma}$ is calibrated using the same procedure as $C_{\mu-\gamma}$ and $C_{\mu 2-\gamma}$. The domain is divided into three groups (see equation 3.52) and individually calibrated with DNS data applying restrictions so no steps or spikes appears in the fitting curve. As done in the $C_{\mu 2-\gamma}$, figure B.7 shows the calibrated coefficient compared with the one calculated solemnly from the DNS and the respective term in which it appears: $\frac{\kappa}{\gamma^{2}} C_{\mu 2-\gamma} P(P, \kappa$ and $\gamma$ extracted from the DNS $)$.

For the region center region $\left(y^{+} \approx 1000\right)$ the calibrated coefficient a more accentuated decay when compared with the DNS profile. This is imposed intentionally to prevent numerical errors propagated by the low values of the $\mathrm{P}$ tensors in this area which hinders the simulation's solution convergence. The $\mathrm{P}$ term, however, is not much affected by this imposed calibration error as the DNS profile and term calculated with the fitting coefficient almost match. 

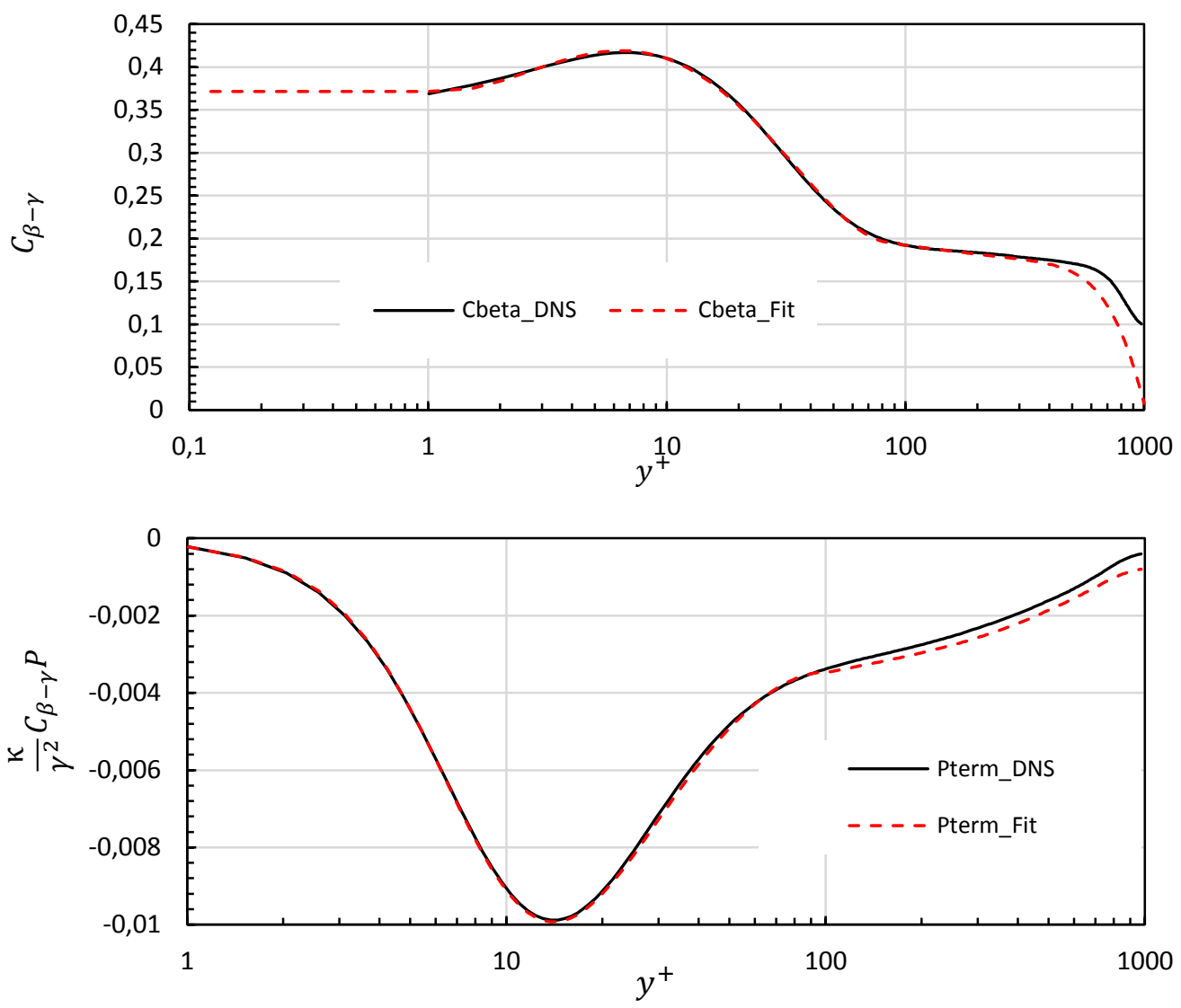

Figure B.7 - Coefficient and P term comparison using DNS data 


\section{Appendix C - Mesh Considerations}

This appendix is divided in two subsections: the first one describes the mesh composition while the second presents the influence of mesh refining.

\section{C.1 Mesh Distribution}

At Chapter 3, it was mentioned that Thompson et al (2016) discussed in their paper that if the flow statistics are not very well converged, errors can be found in the DNS data. Thus, to compare the time average axial field obtained with a RANS modeling, one should employ the velocity field obtained by the solution of the momentum equations with the DNS Reynolds stress tensor components directly inserted at the equations (best possible representation of the tensor). The resulting velocity field was called here as "reference DNS".

In order to determine the "reference" DNS velocity field, a mesh was defined so that no interpolation from the DNS data should be employed. That is, the same mesh as defined in the DNS simulation was employed in the normal $y$ direction. For $R e_{\tau}=1000$ of Thais et al. (2012) at the $y$ direction, 257 elements were defined, with the same non-uniformity as the reference. Here the problem was solved as 2D, i.e., elements at the $x$ direction had to be defined. Since the flow is fully developed, no variation of any variable was expected at the direction, meaning that only 1 point should suffice. However, for simple convenience, and to verify null variation in the axial direction, 4 elements were defined in the present work.

Before continuing, one information must be given, regarding the mesh definition at OpenFoam. In OpenFoam, the position of the center of the element is at half distance between its neighbor faces. Therefore, to be able to follow this mesh's pattern, two more elements were inserted along the $y$ direction at the border of the domain. Without the addition of them, the element's size varied too much, causing divergence. Figure C.1 illustrate the solution taken, where the center of the element is shown in red, and the new elements are yellow. 

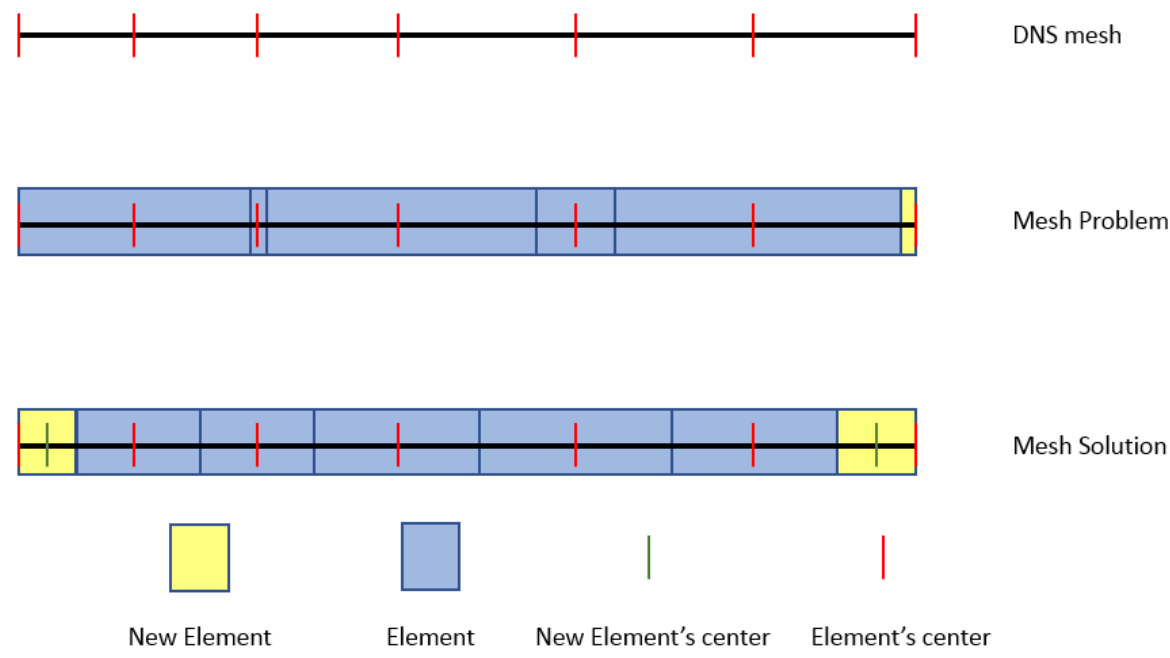

Mesh Solution

Figure C.1 - Mesh definition

\section{C.2 Mesh Refinement}

A grid test was performed to define the mesh in the normal direction. $\kappa-\varepsilon$ Model III, with $R e_{\tau}=1000$ of Thais et al. (2012) was selected to be examined.

Three mesh size were employed in the $y$ direction: 129; 257 and 343. Their elements' positioning followed the same pattern as the original 257 mesh (described at the previous section). The mesh size in the $x$ direction was not varied, and it was verified that no variation was obtained, as expected, in this direction.

Figures C.2 through C.4 show the results obtained for the mean velocity and the Reynolds stress components, as well as for the turbulent kinetic energy and its dissipation rate. All results are coincident, indicating that mesh independent results were obtained.

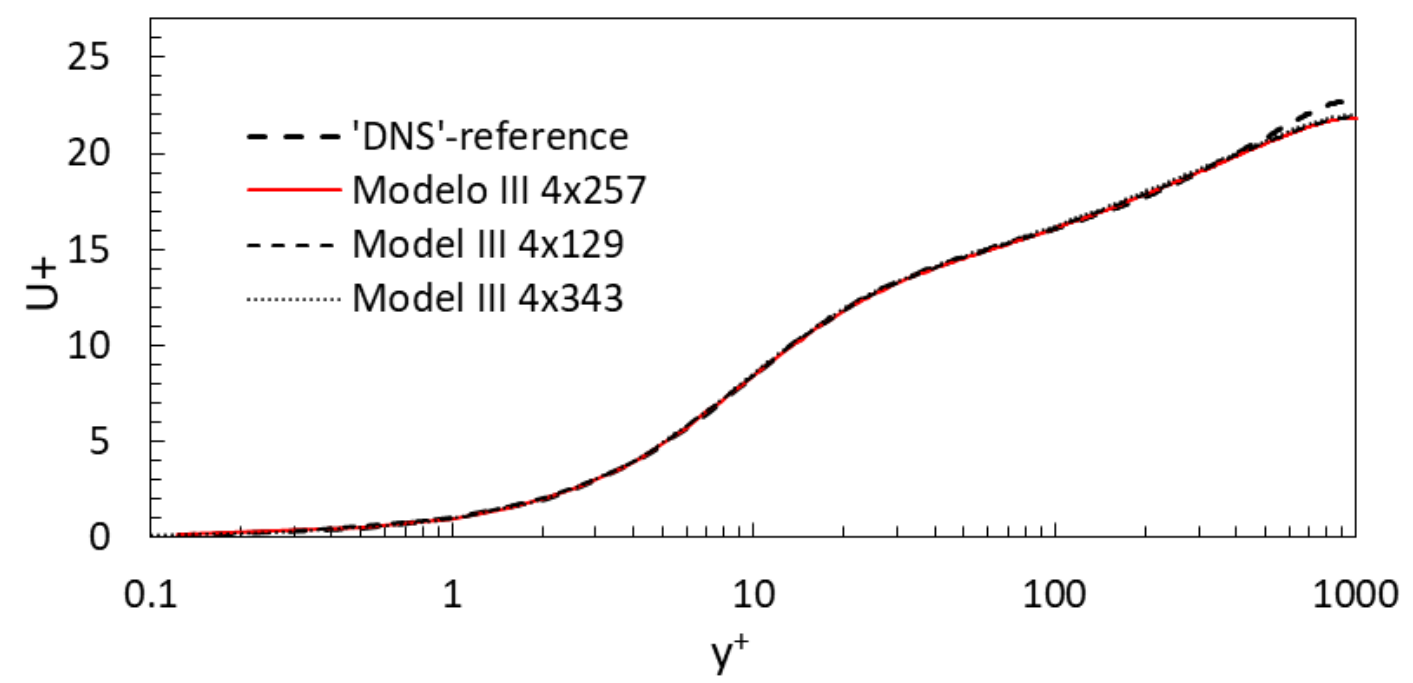

Figure C.2 - Mean axial velocity. Model III. $\boldsymbol{R} \boldsymbol{e}_{\boldsymbol{\tau}}=\mathbf{1 0 0 0}$. Mesh test. 


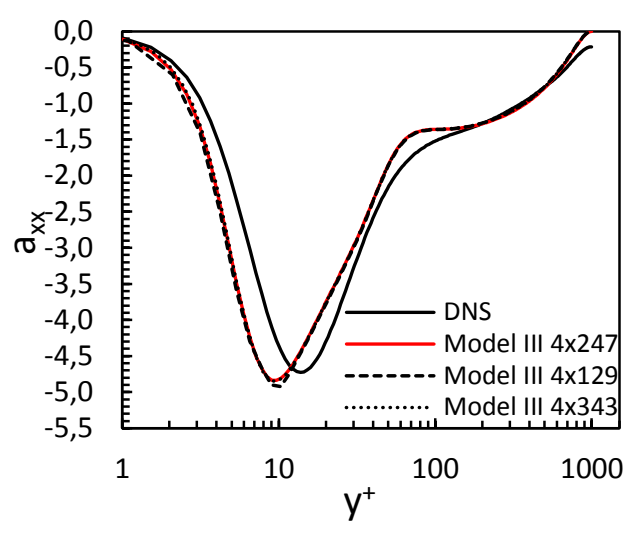

(a) $\boldsymbol{a}_{\boldsymbol{x} x}$

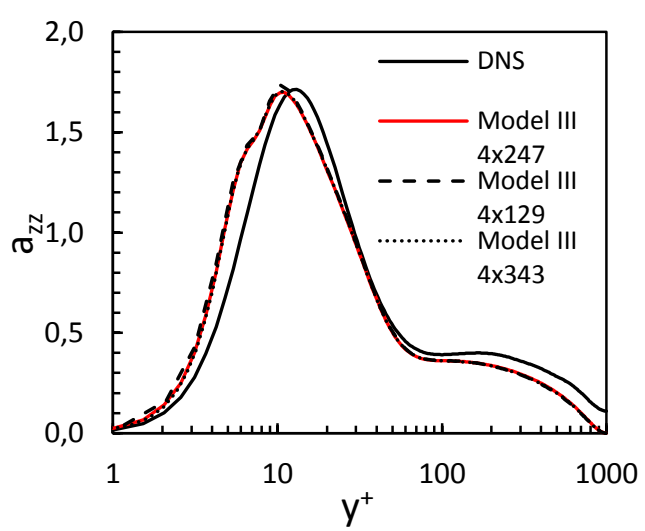

(c) $\boldsymbol{a}_{\mathrm{zz}}$

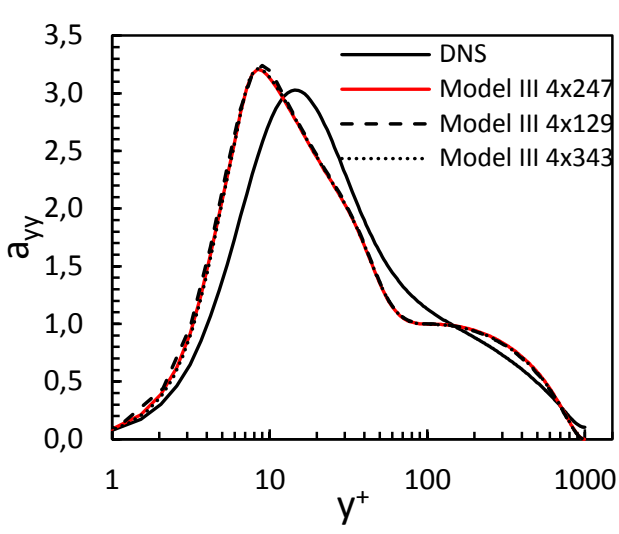

(b) $\boldsymbol{a}_{y y}$

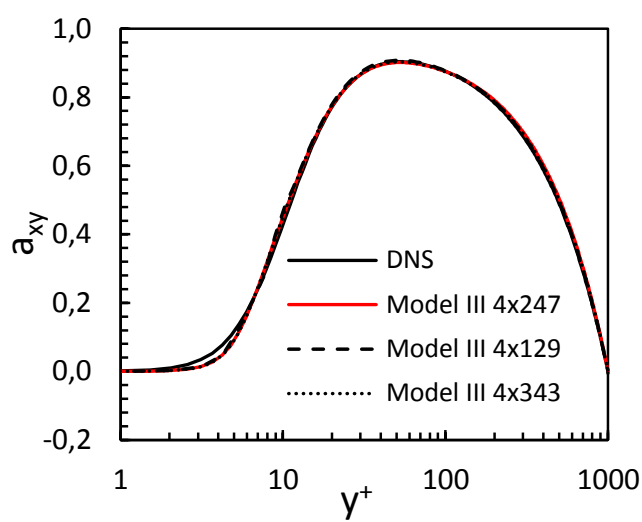

(d) $a_{x y}$

Figure C.3 - Reynolds stress tensor components. Model III. $\boldsymbol{R} \boldsymbol{e}_{\boldsymbol{\tau}}=\mathbf{1 0 0 0}$. Mesh test

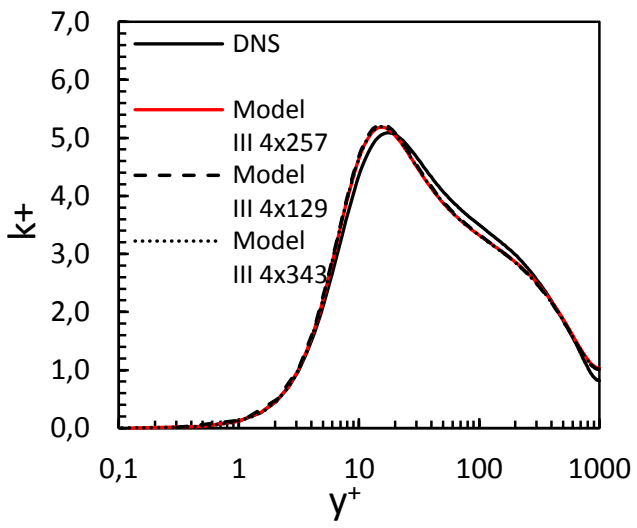

(a) turbulent kinetic energy, $\boldsymbol{\kappa}$

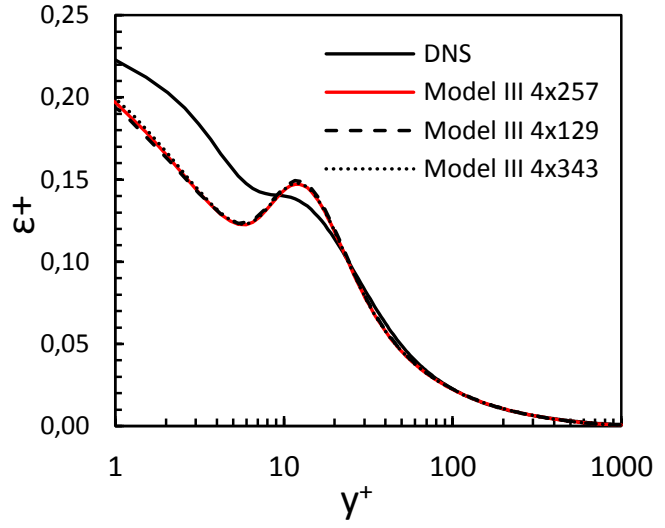

(b) dissipation $\boldsymbol{\varepsilon}$

Figure C.4 - Turbulent kinetic energy and dissipation. Model III. $\boldsymbol{R} \boldsymbol{e}_{\boldsymbol{\tau}}=\mathbf{1 0 0 0}$. Mesh test. 


\section{Appendix D - Influence of $K$ and $\varepsilon$ field into $K-\varepsilon$ NLEVM}

At Section 4.3.1, Model III was selected as the best one which could predict the Reynolds Stress Tensor. However, analyzing the results, it was shown that Model III could not reproduce the DNS data exactly. On the other hand, the analysis a priori performed by Nieckele et al (2016) showed a perfect agreement between Model III and DNS data.

Three types of error related to this misprediction are possible: one associated with the fitting of the damping functions, one associated to the prediction of $\kappa$ and the other one generated from the misevaluation of $\varepsilon$. In this section, therefore, these three causes are analyzed in order to determine the one which most impact the a posteriori solution.

Two types of additional simulations were carried out. At the first one, the $\varepsilon$ field was not numerically determined and the profile obtained from the DNS database was employed directly in the solution, hence, errors associated with $\varepsilon$ predictions and fitting are accounted. For the second case, both DNS fields of $\kappa$ and $\varepsilon$ were directly inserted in the momentum and models' coefficients therefore, only errors of the damping functions' fitting are present. For convenience, they will be referenced as $\varepsilon$ DNS simulation and $\kappa-\varepsilon$ DNS simulation.

These simulations' results are compared with references values of the Reynolds Stress Tensors which were generated by direct calculation using values of $\kappa, \varepsilon, \mathrm{D}_{\mathrm{ij}}$ and $\mathrm{P}_{\mathrm{ij}}$ available in the DNS data base, with the appropriated coefficients and damping functions. These results are referred here as "DNS reference". The resulting field is the best one possible that a simulation could achieve, therefore, it is the best field to use as a reference.

Figure D.1 shows the results from the mentioned simulations compared with the reference field and Model III results. Since the Reynolds Tensor is the variable of major interest, only its components are plotted.

The difference from the DNS reference and the $\kappa-\varepsilon$ DNS simulation curves is attributed to the $D_{i j}$ and $P_{i j}$ which are not the same in each case. Meanwhile, the $\varepsilon$ DNS simulation incorporates the same problem and add the prediction of the $\kappa$ field. 
Finally, the Model III needs to predict both $\mathrm{D}_{\mathrm{ij}}, \mathrm{P}_{\mathrm{ij}}$ and $\kappa$ as well as $\varepsilon$. It is expected and observed, therefore, that the $\kappa-\varepsilon$ DNS simulation is the one which best approach the DNS reference.
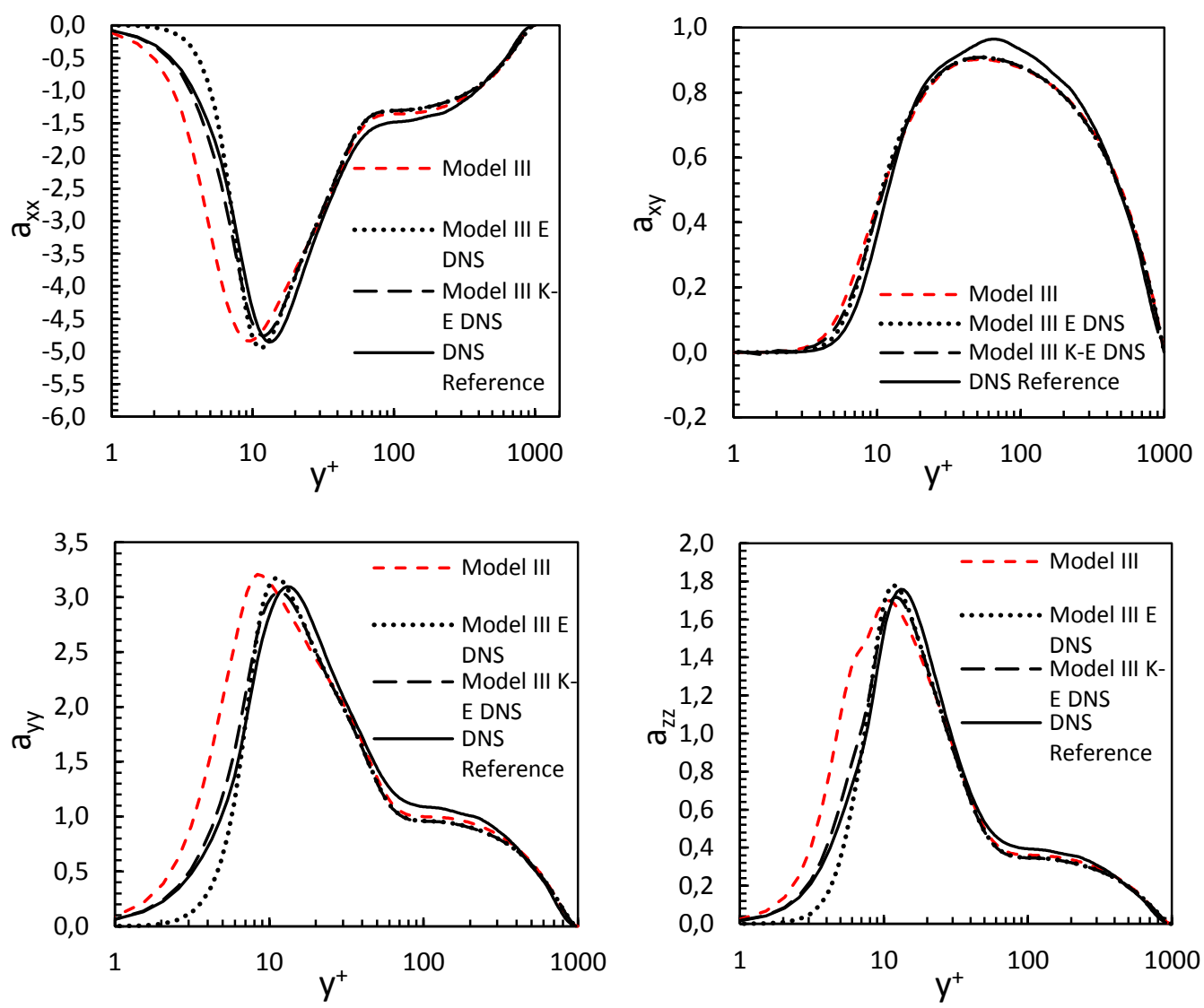

Figure D. 1 - Influence of $\kappa$ and $\varepsilon$ profiles. Model III

Comparing Model III with the $\varepsilon$ DNS simulation profiles it is possible to notice a shift in the normal components profile. It is expected, therefore, that this unwanted shift is caused by the incapacity of the Modified Rodi Mansour model to generate the same $\varepsilon$ field as the DNS. This lack of prediction is also responsible for minor oscillations observed in the azz component.

The $\varepsilon$ field, however, is not the only variable to be blamed since the $\varepsilon$-DNS simulation cannot approach the DNS reference field as the $\kappa-\varepsilon$ DNS simulation. The only difference between these two cases is the $\kappa$ field (calculated in the first and provided by DNS data in the latter). The error associated with this field's calculations is, therefore, responsible for their contrasts. Despite that, the shift problem affecting the components' peak locations is significantly corrected for the $\varepsilon$ DNS simulation. 